BNL- 47380

INFORMAL REPORT

$B N L-47380$

DE92 017696

\title{
AN ORGANIZATIONAL SURVEY OF \\ THE STRATEGIC PETROLEUM RESERVE
}

\author{
Prepared by: \\ Deborah A. Shurberg and Sonja B. Haber \\ Engineering Technology Division \\ Department of Nuclear Energy \\ Brookhaven National Laboratory/Associated Universities, Inc. \\ Upton, New York 11973 \\ January 1992
}

Prepared for:

U.S. Department of Energy

Washington, D.C. 20585

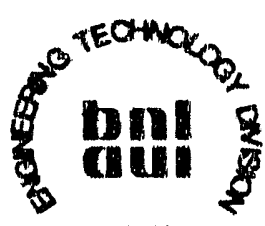

DNE MASTER 


\begin{abstract}
At the request of the management of the Strategic Petroleum Reserve (SPR), an Organizational Survey (OS), identical to the one that has been used prior to Tiger Team Assessments at other Department of Energy facilities, was administered at $\mathrm{EPR}$ independent of a Tiger Team Assessment. The OS measured employees' opinions on subjects such as organizational culture, communication, commitment, group cohesion, coordination, safety, environmental issues, and job satisfaction. The result of this work was a quantitative measure of these variables at the SPR site. SPR management intends to utilize these results in their self-assessment process in preparation for an uponming Tiger Team Assessment. This report presents these results and discusses their interpretation.
\end{abstract}




\section{EXECUTTVE SUMMARY}

An Organizational Survey (OS) was administered at the Strategic Petroleum Reserve (SPR) that queried employees on the subjects of organizational culture, various aspects of communications, employee commitment, work group cohesion, coordination of work, environmental concerns, hazardous nature of worl:, safety and overall job satisfaction. The purpose of the OS administration is to measure in a quantitative and objective way the notion of "culture;" that is, the values, attitudes, and beliefs of the individuals working within the organization. In addition, through the $O S$, a broad sample of individuals can be reached that would probably not be interviewed or observed during the course of a typical assessment. The $O S$ also provides a descriptive profile of the organization at one point in time that can then be cornpared to a profile taken at a different point in time to assess changes in the culture of the organization.

The OS administration at SPR was somewhat different from the prior twelve administrations which have occurred at Department of Energy (DOE) facil . es. The prior surveys have all occurred in conjunction with a Tiger Tearn Assessment. The SPR survey administration occurred at the request of SPR management at a time approximately five months prior to the start of their Tiger Team Assessment. SPR management intends to utilize the survey results in their pre-assessment. Another survey administration will be conducted at a later date using a similar method of administration.

The SPR is operated for DOE by Boeing Corporation. The OS was administered in groups at all eight SPR locations to both Boeing and DOE employees. Of the 12.24 employees at SPR, 879 completed the survey, yielding a response rate of 71.8 percent. A response rate of 64.0 percent was obtained for the Boeing employees and 94.8 percent for the DOE employees. The distribution of responses was varied across Boeing Departments with the lowest response rate of 50.9 percent in the Operations and Maintenance Department, and the highest response rate of 116.7 percent in the Project Planning and Control Department. All data from the $O S$ is presented in group summaries, by organization, location, department, employee category, and supervisory level. Statistically significant differences between groups are identified and discussed.

The overall results obtained from the sample of employees who participated in the OS at SPR indicate they believe that approval-, conventionalism-, and dependence-type behaviors are expected of them in order to be successful within the organization. Some emphasis is also placed on producing quality work in a constructive and creative manner. The low mean values obtained on the Communication Scales are further evidence for a passive-defensive cultural type. Mean scores on the Coordination and Hazard Scales were also relatively low, indicating that SPR employees do not perceive work to be highly coordinated or hazardous. However, those attributes which are important to safety are believed to be helpful, as indicated by the high mean values obtained on the Safery Scale.

No differences were found between the DOE and Boeing Organizations, indicating that the description for the overall SPR organization is appropriate to both organizations. Some differences were obtained between the various SPR locations. These differences portray the New Orleans Location as a consistent outlier from the other locations, especially on those scales which relate to the degree of hazardous or the environmental consequences of one's work. These differences are not surprising due to the different functional role the New Orleans Location plays within the SPR organization. While other differences do exist, they are not nearly as consistent or extreme. The location which appears to be the most different from the New Orleans Location is the Sulphur Mines Location. 
Few differences were found between employee categories and those which did exist appear to reflect differences in job functions. Differences between supervisory levels were more numerous than might be expected, altnough they were consistent with results found at other DOE facilities.

Differences between groups in the Boeing Organization were few and appeared to reflect functional differences in all analyses except the supervisory level analysis. Differences between supervisory levels within the Boeing Organization were even more numerous than those found between levels for the overall SPR organization.

Only one difference was found between any of the DOE groups analyzed. This finding suggests that the DOE Organization is very homogeneous with respect to those issues assessed using the OS. 


\section{CONTENTS}

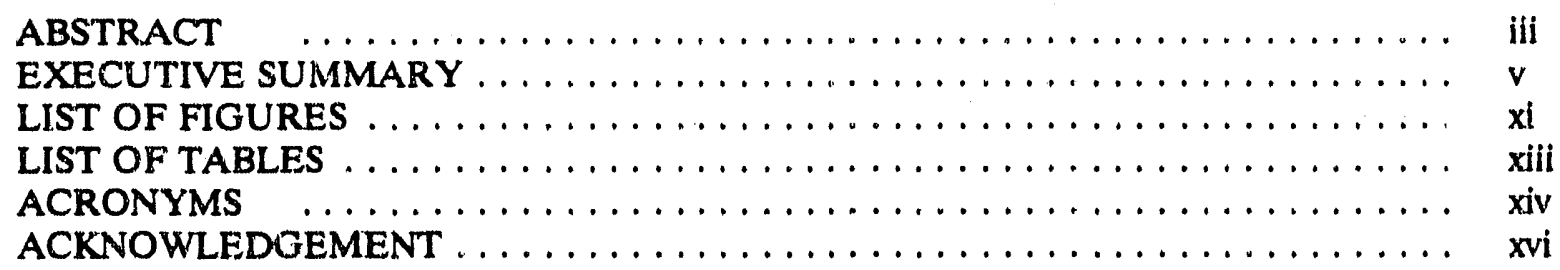

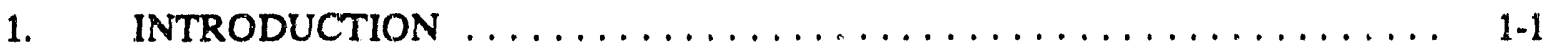

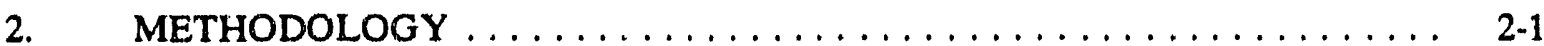

3. ORGANIZATIONAL DESCRIPTION $\ldots \ldots \ldots \ldots \ldots \ldots \ldots \ldots \ldots \ldots$

4. ORGANIZATIONAL SURVEY SCALES AND RESULTS . . . . . . . . . 4 4-1

4.1 Organizational Survey Scale Descriptions $\ldots \ldots \ldots \ldots \ldots \ldots \ldots \ldots$. $\ldots \ldots$

4.1.1 Organizational Culture Inventory $\ldots \ldots \ldots \ldots \ldots \ldots \ldots \ldots$ 4-1

4.1.2 Communication Scales .................... 4-3

4.1.3 Commitment Scale . . . . . . . . . . . . . . . . . . . 4-4

4.1.4 Cohesion Scale ....................... 4.4

4.1.5 Coordination Scale ... . . . . . . . . . . . . . . . . . 4 4-4

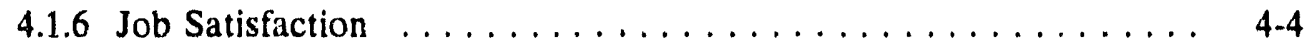

4.1.7 Hazard Scale . . . . . . . . . . . . . . . . . . . 4 4-4

4.1 .8 Safety Scale . . . . . . . . . . . . . . . . . . . . 4-4

4.1.9 Environment, Safety, and Health Questions . . . . . . . . . 4-5

4.2 Overall Results on the OS Scales for SPR $\ldots \ldots \ldots \ldots \ldots \ldots$

4.2.1 Organizational Culture Inventory Results $\ldots \ldots \ldots \ldots \ldots \ldots . . .4 .5$

4.2.2 Communication Scales Results . . . . . . . . . . . . . . 4. 4-6

4.2.3 Results for Additional Scales ... . . . . . . . . . . . . 4.6

4.2.4 Environment, Safety and Health Questions Results . . . . . . . . 4-6

4.2 .5 Summary $\ldots \ldots \ldots \ldots \ldots \ldots \ldots \ldots \ldots \ldots \ldots . \ldots \ldots \ldots$

4.3 Differences Between Organizations on the OS Scales $\ldots \ldots \ldots \ldots \ldots 4.8$

4.3.1 Differences Between Organizations on the $\mathrm{OCl} \ldots \ldots \ldots \ldots \ldots . .4$

4.3.2 Differences Between Organizations on the Communication Scales 4-8

4.3.3 Differences Between Organizations on the Additional Scales . . . 4. 4-8

4.3.4 Differences Between Organizations on the Environment, Safety, and Health Questions . . . . . . . . . . . . . . . . . . . . 4.8

4.3.5 Summary . . . . . . . . . . . . . . . . . . . . . . 4 4-8 
4.4 Differences Between Locations on the OS Scales $\ldots \ldots \ldots \ldots \ldots \ldots .4 .8$

4.4.1 Differences Between Locations on the OCI Scales . . . . . . . . 4.8

4.4.2 Differences Between Locations on the Communication Scales . . . . . . . . . . . . . . . . . . . . . . . . . 4.9 4

4.4.3 Differences Between Locations on the Additional Scales ...... 4. 4-9

4.4.4 Differences Between Locations on the Environment, Safety, and

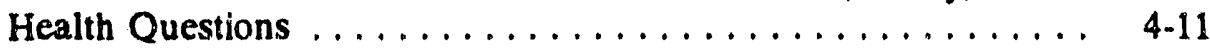

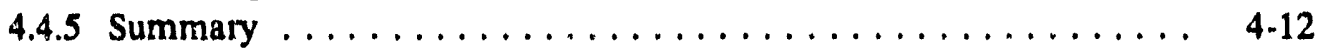

4.5 Differences Between Employee Categories on the OS Scales . . . . . . . 4-13

4.5.1 Differences Between Employee Categories on the OCI Scales ... 4-13

4.5.2 Differences Between Employee Categories on the Communication Scales ........................... 4-13

4.5.3 Differences Between Employee Categories on the Additional Scales ............................ 44-14

4.5.4 Differences Between Employee Categories on the Environment, Safety, and Health Questions $\ldots \ldots \ldots \ldots \ldots \ldots \ldots \ldots \ldots$ 4-14

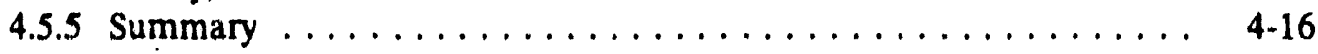

4.6 Differences Between Supervisory Levels on the OS Scales . . . . . . . . 4.16

4.6.1 Differences Between Supervisory Levels on the OCI Scales .... . 4-16

4.6.2 Differences Between Supervisory Levels on the Communication Scales ............................. 4-19

4.6.3 Differences Between Supervisory Levels on the Additional Scales . 4-20

4.6.4 Differences Between Supervisory Levels on the Environment, Safety, and Health Questions ................. 4-21

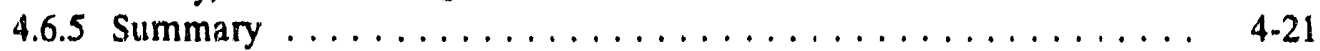

4.7 Differences Between the Boeing Departments on the OS Scales .... . $4-22$

4.7.1 Differences Between the Boeing Departments on the OCI Scales . $\quad 4.22$

4.".2 Differences Between the Boeing Departments on the Communication Scales .................... 4-22

4.7.3 Differences Between the Boeing Departments on the Additional Scales .......................... 4-22

4.7.4 Differences Between the Boeing Departments on the Environment, Safety, and Health Questions $\ldots \ldots \ldots \ldots \ldots \ldots \ldots \ldots \ldots \ldots . \ldots \ldots$

4.7 .5 Summary $\ldots \ldots \ldots \ldots \ldots \ldots \ldots \ldots \ldots \ldots \ldots \ldots \ldots . . \ldots \ldots \ldots$

4.8 Differences Between DOE Departments on the OS Scales ... . . . $4-24$ 


\section{CONTENTS (Cont'd.)}

Page

4.8.1 Differences Between DOE Departments on the OCI Scales . . . . 4-24

4.8.2 Differences Between DOE Departments on the Communication Scales . . . . . . . . . . . . . . . . . . . . . . . . . . 4 4-24

4.8.3 Differences Between DOE Departments on the Additional Scales 4-24

4.8.4 Differences Between DOE Departments on the Environment, Safety, and Health Questions .................. 4-24

4.8.5 Summary ........................ 4-25

4.9 Differences Between the Boeing Employee Categories on the OS Scales 4-25

4.9.1 Differences Between the Boeing Employee Categories on the OCI Scales . . . . . . . . . . . . . . . . . . . . . . . .

4.9.2 Differences Between the Boeing Employee Categories on the Communication Scales ...................... 4-25

4.9.3 Differences Between the Boeing Employee Categories on the Additional Scales . . . . . . . . . . . . . . . . . . . . 4-25

4.9.4 Differences Between the Boeing Employee Categories on the Environment, Safety, and Health Questions . . . . . . . . . 4 4-26

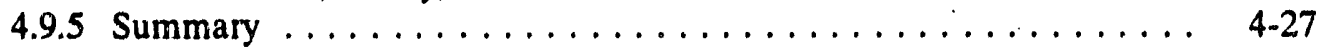

4.10 Differences Between DOE Employee Categories on the OS Scales ... 4-27

4.10.1 Differences Between DOE Employee Categories on the OCI Scales . . . . . . . . . . . . . . . . . . . . . .

4.10.2 Differences Between DOE Employee Categories on the Communication Scales ....................

4.10.3 Differences Between DOE Employee Categories on the

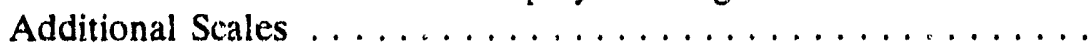

4.10.4 Differences Between DOE Employee Categories on the Environmeni, Safety, and Health Questions . . . . . . . . . . . 4 4-28

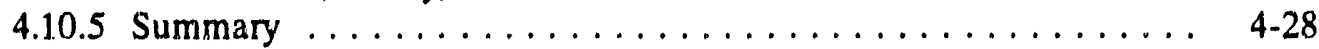

4.11 Differences Berween Boeing Supervisory Levels on the OS Scales . . . . 4-28

4.11.1 Differences Between Boeing Supervisory Levels on the OCI Scales ............................

4.11.2 Differences Between Boeing Supervisory Levels on the

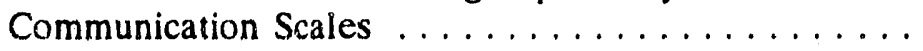

4.11.3 Differences Between Boeing Supervisory Levels on the Additional Scales . . . . . . . . . . . . . . . . . . 44-31

4.11.4 Differences Between Boeing Supervisory Levels on the Environment, Safety, and Health Questions . . . . . . . . . 4-34

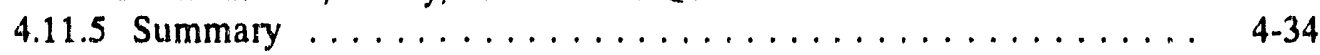

4.12 Differences Between DOE Supervisory Levels on the OS Scales . . . . 4-34 


\section{CONTENTS (Cont'd.)}

4.12.1 Differences Between DOE Supervisory Levels on the

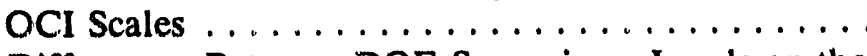

4.12.2 Differences Between DOE Supervisory Levels on the

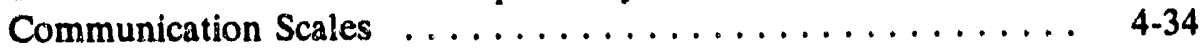

4.12.3 Differences Between DOE Supervisory Levels on the Additional Scales . . . . . . . . . . . . . . . . . 4-34

4.12.4 Differences Between DC.E Supervisory Levels on the

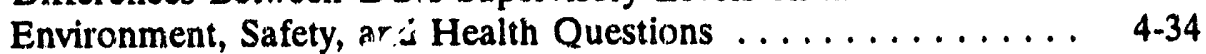

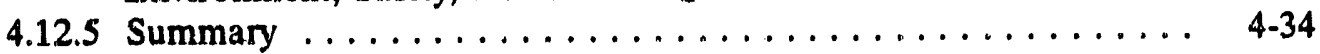

5. CONCLUSIONS $\ldots \ldots \ldots \ldots \ldots \ldots \ldots \ldots \ldots \ldots \ldots \ldots \ldots \ldots \ldots \ldots \ldots$

6. REFERENCES $\ldots \ldots \ldots \ldots \ldots \ldots \ldots \ldots \ldots \ldots \ldots \ldots \ldots \ldots \ldots$

Appendix A: $\overline{\mathbf{x}}$ Values Obtained for Each SPR Organization on the OS Scales ..... A-1

Appendix B: Comparison of $\overline{\mathrm{x}}$ for SPR Overall to $\overline{\mathrm{x}} \mathrm{S}$ obtained by Boeing and

DOE on the OCI Scales $\ldots \ldots \ldots \ldots \ldots \ldots \ldots \ldots \ldots \ldots \ldots \ldots$

Appendix C: Comparison of $\bar{x}$ for SPR Overall to $\bar{x} s$ Obtained by Boeing and

DOE on Communication Scales

Appendix D: Comparison of $\overline{\mathrm{x}}$ for SPR Overall to $\overline{\mathrm{x}} \mathrm{S}$ Obtained by Boeing and

DOE on Additional Scales

Appendix E: Comparison of $\bar{x}$ for SPR Overall to $\bar{x} S$ Obtained by Boeing and

DOE on the Environment, Safety and Health Questions

Appendix $\mathrm{F}: \quad \overline{\mathrm{x}}$ Values Obtained for SPR Locations on the OS Scales $\ldots \ldots \ldots$ F-1

Appendix G: $\overline{\mathrm{x}}$ Values Obtained for SPR Employee Categories on the OS Scales ....

Appendix H: $\quad \overline{\mathrm{x}}$ Values Obtained for SPR Supervisory Levels on the OS Scales .....

Appendix I: $\quad \overline{\mathrm{x}}$ Values Obtained for Boeing Departments on the OS Scales $\ldots . \ldots$ I-1

Appendix J: $\quad \overline{\mathbf{x}}$ Values Obtained for DOE Departments on the OS Scales $\ldots \ldots . . . \mathrm{J}-1$

Appendix K: $\quad \overline{\mathrm{x}}$ Values Obtained for Boeing Employee Categories on the OS Scales . . K-1

Appendix L: $\quad \overline{\mathrm{x}}$ Values Obtained for DOE Employee Categories on the OS Scales $\ldots \quad$ L-1

Appendix M: $\overline{\mathrm{x}}$ Values Obtained for Boeing Supervisory Levels on the OS Scales . . . M-1

Appendix N: $\overline{\mathrm{x}}$ Values Obtained for DOE Supervisory Levels on the OS Scales $\ldots . . \quad \mathrm{N}-1$ 


\section{LIST OF FIGURES}

Page

4.1 Overall mean values on the OCI scales for SPR . . . . . . . . . . . $4-5$

4.2 Overall mean values on the communication scales for SPR . . . . . . . . . . 4-6

4.3 Overall mean values on additional scales for SPR . . . . . . . . . . . . . 4-7

4.4 Overall mean values on environment, safety, and health questions for SPR ... 4-7

4.5 Significant differences between SPR locations on the cohesion scale $\ldots \ldots \ldots$. . . 4

4.6 Significant differences between SPR locations on the hazard scale . . . . . . . . 4-10

4.7 Significant differences between SPR locations on the safety scale $\ldots \ldots \ldots \ldots .4-10$

4.8 Significant differences between SPR locations on the coordination scale . . . . 4-10

4.9 Significant differences between SPR locations on the offsite consequences scale . 4-11

4.10 Significant differences between SPR locations on the onsite consequences scale . 4-11

4.11 Significant differences between SPR locations on the management emphasis

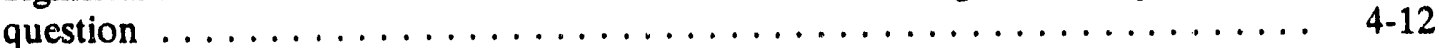

4.12 Significant differences between SRR locations on the employee awareness question 4-12

4.13 Significant differences between SPR employee categories on the communication-

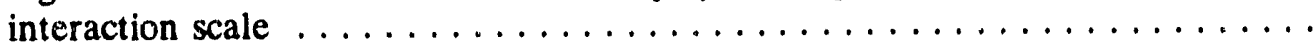

4.14 Significant differences between SPR employee categories on the hazard scale . .

4.15 Significant differences between SPR employee categories on the offsite consequences scale $\ldots \ldots \ldots \ldots \ldots \ldots \ldots \ldots \ldots \ldots \ldots \ldots \ldots \ldots \ldots$ 4-15

4.16 Significant differences between SPR employee categories on the onsite consequences question $\ldots \ldots \ldots \ldots \ldots \ldots \ldots \ldots \ldots \ldots \ldots \ldots \ldots \ldots$ 4-15

4.17 Significant differences between SPR employee categories on the management

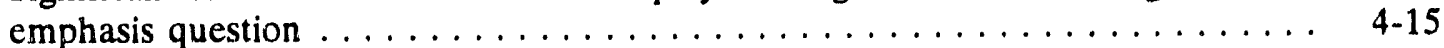

4.18 Significant differences between SPR employee categories on the employee

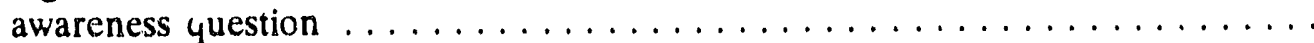

4.19 Significant differences between SPR supervisory levels on the humanistic-

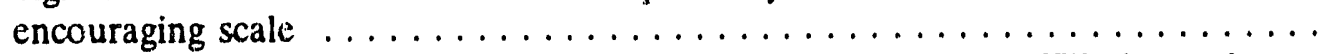
Significant differences between SPR supervisory levels on the affiliative scale . . Significant differences between SRR supervisory levels on the conventional scale Significant differences between SPR supervisory levels on the achievement scale Significant differences between SPR supervisory levels on the self-actualizing scale

4.24 Significant differences between SPR supervisory levels on the communication-trust

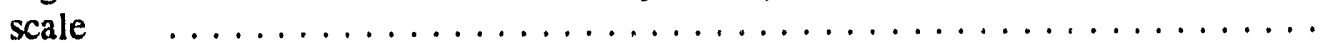

4.25 Significant differences between SPR supervisory levels on the communication-

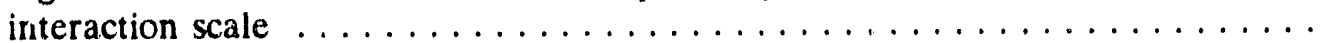
Significant differences between SPR supervisory levels on the communication-

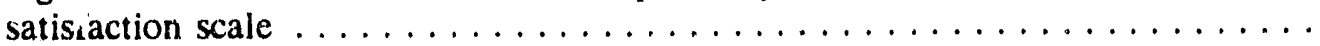
Significant differences between SPR supervisory levels on the commitment scale Significant differences between SPR supervisory levels on the cohesion scale ... 
4.30 Significant differences between Boeing departments on the hazard scale .....

4.31 Significant differences between Boeing departments on the offsite consequences

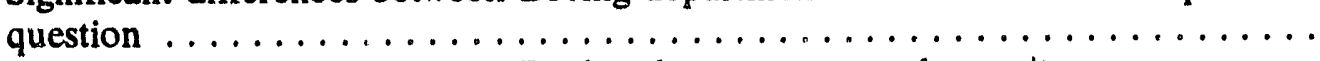

4.32 Significant differences between Boeing departments on the onsite consequences

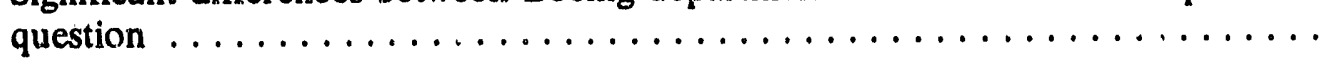

4.33 Significant differences between DOE departments on the onsite consequences

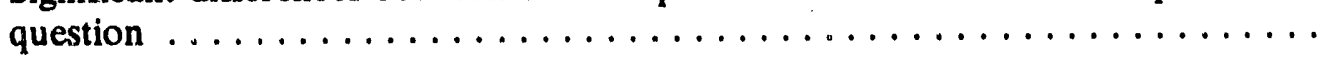

4.34 Significant differences between Boeing employee categories on the hazard scale

4.35 Significant differences between Boeing employee categories on the offsite

4.36 Significant differences between Boeing employee categories on the onsite

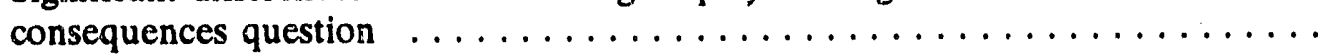

4.37 Significant differences between Boeing supervisory levels on the humanistic-

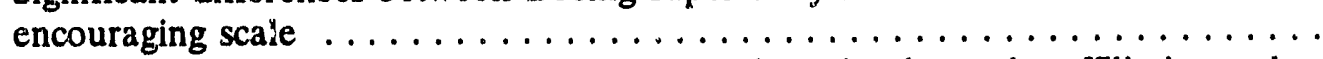

4.38 Significant differences between Boeing supervisory levels on the affiliative scale . Significant differences between Boeing supervisory levels on the conventional scale Significant differences between Boeing supervisory levels on the dependent scale Significant differences between Boeing supervisory levels on the achievement scale 4-30

4.42 Significant differences between Boeing supervisory levels on the self-actualizing

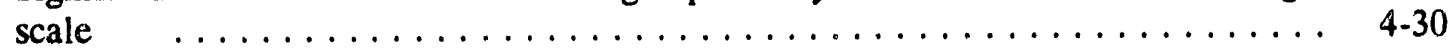

4.43 Significant differences between Boeing supervisory levels on the communication-

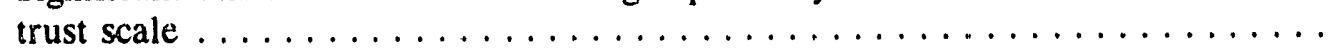

4.44 Significant differences between Boeing supervisory levels on the communication-

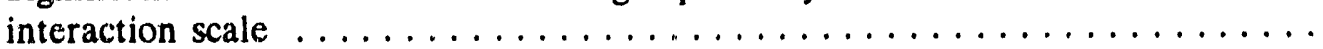

4.45 Significant differences between Boeing supervisory levels on the communication-

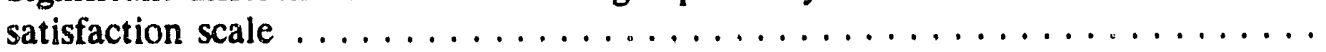

Significant differences between Boeing supervisory levels on the commitment scale Significant differences between Boeing supervisory levels on the cohesion scale . Significant differences between Boeing supervisory ievels on the safety scale ... 


\section{LIST OF TABLES}

Page

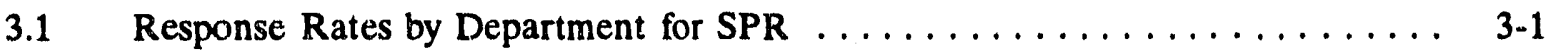

3.2 Distribution of Employee Categories at SPR $\ldots \ldots \ldots \ldots \ldots \ldots \ldots \ldots \ldots \ldots$

3.3 Distribution of Supervisory Levels at SPR $\ldots \ldots \ldots \ldots \ldots \ldots \ldots \ldots \ldots \ldots \ldots$

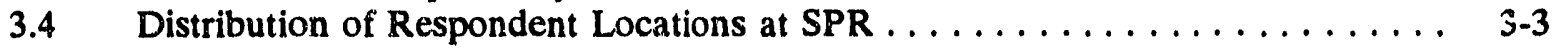

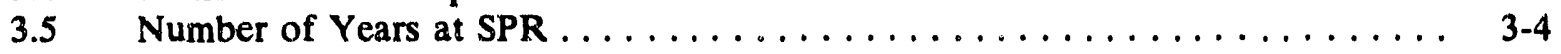

3.6 Educational , evel of SPR Respondents $\ldots \ldots \ldots \ldots \ldots \ldots \ldots \ldots \ldots \ldots \ldots$

3.7 Modal Educational Level and Mean Number of Years at SPR Overall and for

Each Organization $\ldots \ldots \ldots \ldots \ldots \ldots \ldots \ldots \ldots \ldots \ldots \ldots \ldots \ldots \ldots$ 


\section{ACRONYMS}

SPR

BOE

DOE

Strategic Petroleum Reserve

Organization

Boeing Petroleum Services

U.S. Department of Energy

\section{Departments}

Boeing:

BM

Business Management

ENG

MAT

O\&M

PM

PPC

SFP

TA

Engineering

Material

Operations \& Maintenance

Project Management

Project Planning and Control

Security and Fire Protection

Technical Assistance

DOE:

$\mathrm{E} \& \mathrm{C}$

ESH

$M \& A$

OTH

OPR

PMO

Engineering \& Construction

Environment, Safety, and Health Division/Technical Assurance

Management \& Administration

Other

Operations

Project Manager's Office

\section{Location}

$\mathrm{BC}$

BH

$\mathrm{BM}$

NO

SJ

SM

WH

WI

Bayou Choctaw

Big Hill

Bryan Mound

New Orleans

Saint James

Sulphur Mines

West Hackberry

Weeks Island

\section{Employee Category}

EXMT

HOUR

NEA

NEB

Exempt (Boeing or DOE)

Hourly (Boeing)

Non-Exempt - Administrative Support (DOE)

Non-Exempt (Boeing) 
ACRONYMS (Continued)

NET

OTH

Non-Exempt - Technical (DOE)

Other

Supervisory Level

NSUP

SUP

Non-Supervisor/Non-Manager

Supervisor/Manager

Survey Srales

Cl Humanistic-Encouraging

C2. Affliative

C3 Approval

C4 Conventional

C5 Dependent

C6 Avoidance

C7 Oppositional

C8 Power

C9 Competitive

C10 Perfectionistic

C11 Achievement

C12 Self-Actualizing

ACCURACY (CMA)

AWARENESS (EMA)

COHESION (COH)

COMMITMT (COT)

COORD (COD)

EMPHASIS (MGE)

HAZARD (HAZ)

INTERACT (CMI)

JOBSAT (JOB)

OFFSITE (OFF)

ONSITE (ONS)

SAFETY (SAF)

SATISFAC (CMS)

TRUST (CMT)
Perceived Accuracy of Communications

Employee Awareness of Worhplace Hazards

Cohesion of Work Gr oup

Organizational Cummitment

Coordination

Management Emphasis of Environmental Issues

Perceived Hazardous Nature of Work

Desirability of Interaction with Others

Overall Job Satisfaction

Consequence to Offsite Environment

Consequence to Onsite Environment

Attention to Safety

Satisfaction with Communications

Trust in Communications 


\section{ACKNOWLEDGEMENT}

The authors wish to thank the Department of Energy (DOE) staff that have been supportive and facilitative of this work, Mark Gilbertson, and Larry Weiner, as well as the Strategic Petroleum Reserve (SPR) for their cooperation in this effort, in particular, Dick Mitchell and Red O'Laughlin, for their help in arranging the logistics and details of the survey administration.

We also extend our appreciation to the efforts of our Brookhaven Nattional Laboratory colleagues, Michael Barriere, for his help in administering the survey, David Stock, for his statistical assistance and expertise and Kathleen Nasta for her help in preparing this manuscript. In addition, the review, comments, and insights provided by James Higgins were also very much appreciated. 


\section{INTRODUCTION}

An Organizational Survey (OS) was administered at the Strategic Petroleum Reserve (SPR) that queried employees on the subjects of organizational culture "various aspects of communication, employee commitment to SPR, work group cohesion, coordination of work, environmental concerns, hazardous nature of work, safety, and overall job satisfaction. A description of each of the scales used to assess these subjects is discusised bellow.

The primary purpose of administering the survey was to attempt to measure, in a quantitative and objective way the notion of "organizational culture," that is, the values, attitudes, and beliefs of the individuals working within the organization. In particular, those aspects of the working environment which are believed to be important influences on the operations of a facility and on the safety issues relevant to the organization were assessed.

In addition, by conducting a survey, a broad sampling of the individuals in the organization can be obtained. This is especially important when the survey is utilized in conjunction with an assessment or inspection team which typically has only a limited amount of resources $t ?$ address many issues. The OS provides a broad and comprehensive picture of the organization by querying a much larger number of individuals than could be reached through the assessment team alone.

Finally, the OS provides a descriptive profile of the organization at one point in time. This profile can then be used as a baseline point against which comparisons of other points in time can be made. Such comparisons may prove valuable and would help to assess changes in the organizational culture. Comparisons of profiles can also be made across similar facilities. 


\section{METHODOLOGY}

The Organizational Survey (OS) was administered to the employees of SPR in groups. The surveys were administered between November 4 and 8,1991 . Included with the survey was a cover letter explaining the purpose for the survey administration. Prior to the survey administration, a memorandum from the Director of SPR was circulated. This memorandum encouraged employees to complete the survey and contained the times at which various groups of employees were to take the survey. A demographics sheet attached to the survey requested information pertainir; to the organization the respondent was a member of, the department in which the respondent was located, the number of years they had been working at SPR, the site which they were located at, their employee category, and their supervisory and educational leveis.

Two individuals familiar with the OS were at SPR during the survey administration in order to distribute the surveys and to answer any questions employees had. A total of 879 surveys were completed, for a response rate of 71.8 percent. The surveys were taken from SPR for data entry and analysis.

Overall means, standard errors, anu standard deviations were computed for each scale assessed in the OS. A one way analysis of variance was also performed on each OS scale using the scale score as the dependent variable and separate analyses using organization, department, employee category, locations, and supervisory level as the independent variables. In order to control the false positive rate (Type I error rate), the Bonferroni correction was applied to all the analyses of variance performed for each independent variable. Since there were 26 one-way analyses of variance for each independent variable, the significance level for each was reduced to $.05 / 26=.0019$. Where the analysis of variance showed a significant difference among the group means at the .0019 level, a Tukey HSD (Honestly Significant Difference) (Hays, 1988) procedure was applied to identify those means that were statistically significantly different from each other. Consequently, the results that are reported to be significantly different from each other represent a very conservative approach in the interpretation of the data analysis performed.

Included in this report are the overall results for SPR on each of the scales used in the OS. In addition, any statistically significant differences between organizations, departments, employee categories, locations, and supervisory levels are also presented. 


\section{ORGANIZATIONAL DESCRIPTION}

At the Strategic Petroleum Reserve (SPR), two organizations, Boeing and DOE participated in the OS. Each organization identifies its organizational units as departments. The demographics sheet used in the administration of the OS included eight departments for Boeing and five departments for DOE. The departments and their abbreviations, as used in this report, are presented in Table 3.1. Also presented in this table are the response rates for the two organizations, as well as for each of the Boeing Departments. The response rate is computed by dividing the number of surveys returned by the number of employees in that department. The DOE Organization obtained the higher response rate of 94.8 percent. Within the Boeing Organization, the highest response rate obtained was in the Project Planning and Control Department, which had a response rate of 116.7 percent. The lowest response rate was in the Operations and Maintenance Department which had a response rate of 59.9 percent. The overall response rate obtained at SPR was 71.8 percent.

Table 3.1. Response Rates by Department for SPR

\begin{tabular}{|l|c|c|c|}
\hline \multicolumn{1}{|c|}{ Organization/Department } & $\begin{array}{c}\text { No. } \\
\text { Responses }\end{array}$ & $\begin{array}{c}\text { No. } \\
\text { Employees }\end{array}$ & $\begin{array}{c}\text { Response } \\
\text { Rate }\end{array}$ \\
\hline \hline Boeing (BOE) & 697 & 1089 & 64.0 \\
\hline Operations \& Maintenance (O\&M) & 264 & 525 & 50.9 \\
\hline Project Planning \& Control (PPC) & 28 & 24 & 116.7 \\
\hline Engineering (ENG) & 122 & 157 & 77.7 \\
\hline Technical Assurance (TA; & 63 & 77 & 81.2 \\
\hline Security \& Fire Protection (SFP) & 30 & 49 & 61.2 \\
\hline Business Management (BM) & 92 & 113 & 81.4 \\
\hline Material (MAT) & 92 & 136 & 67.6 \\
\hline Project Management (PM) & 6 & 8 & 75.0 \\
\hline Department of Energy (DOE) & 128 & 135 & 94.8 \\
\hline Unknown & 54 & $\ldots$ & $\ldots$ \\
\hline
\end{tabular}

SPR employees were also given six employee categories on the demographics sheet in which to categorize themselves, some of which were specific to the organization in which they worked. Table 3.2 presents these employee categories, and their abbreviations. This table also presents the percent of the Boeing sample, the DOE sample, and the total sample each of the employee categories represent. Within Boeing, the Exempt Employee Category accounts for the largest number of respondents. This is also true within the DOE Organization, and within SPR overall for those people who responded to the survey. 
Table 3.2. Distribution of Employee Categories at SPR

\begin{tabular}{|c|c|c|c|c|c|c|}
\hline \multirow[b]{2}{*}{$\begin{array}{l}\text { Enployee } \\
\text { Category }\end{array}$} & \multicolumn{2}{|c|}{ Boeing } & \multicolumn{2}{|c|}{ DOE } & \multicolumn{2}{|c|}{ Overall } \\
\hline & $\begin{array}{l}\text { No. } \\
\text { Resps. }\end{array}$ & $\begin{array}{l}\text { \%o Boeing } \\
\text { Sample }\end{array}$ & $\begin{array}{l}\text { No. } \\
\text { Resps. }\end{array}$ & $\begin{array}{l}\text { \% DOE } \\
\text { Sample }\end{array}$ & $\begin{array}{l}\text { No. } \\
\text { Resps. }\end{array}$ & $\begin{array}{l}\text { \% Total } \\
\text { Sample }\end{array}$ \\
\hline $\begin{array}{l}\text { Exempt (Boeing or DOE) } \\
\text { (EXMT) }\end{array}$ & 314 & 45.0 & 44 & 34.0 & 374 & 42.5 \\
\hline $\begin{array}{l}\text { Non-Exempt (Boeing) } \\
\text { (NEB) }\end{array}$ & 174 & 25.0 & 0 & 0.0 & 181 & 20.6 \\
\hline $\begin{array}{l}\text { Non-Exempt Administrative } \\
\text { Support (DOE) (NEA) }\end{array}$ & 0 & 0.0 & 33 & 25.8 & 34 & 3.9 \\
\hline $\begin{array}{l}\text { Non-Exempt Texhnical } \\
\text { (DOE) (NET) }\end{array}$ & 0 & 0.0 & 11 & 8.6 & 12 & 1.4 \\
\hline Hourly (Boeing) (HOUR) & 150 & 21.5 & 0 & 0.0 & 1.55 & 17.6 \\
\hline Other (OTH) & 9 & 1.3 & 3 & 2.3 & 12 & 1.4 \\
\hline Unknown & 50 & 7.1 & 37 & 28.9 & 111 & 12.6 \\
\hline
\end{tabular}

Note: Numbers do not total overall due to individuals who indicated employee category but not organization.

The Organizational Survey (OS) demographics questions used at SPR also provided two categories of supervisory levels by which an employee could identify him/herself. Table 3.3 presents the percent of the Boeing, the DOE, and the total SPR sample which classified themselves into each of the two categories.

Table 3.3. Distribution of Supervisory Levels at SPR

\begin{tabular}{||l|c|c|c|c|c|c|}
\hline & \multicolumn{2}{|c|}{ Boeing } & \multicolumn{2}{c|}{ DOE } & \multicolumn{2}{c|}{ Overall } \\
\cline { 2 - 7 } \multicolumn{1}{|c|}{ Supervisory Level } & $\begin{array}{c}\text { No. } \\
\text { Resps. }\end{array}$ & $\begin{array}{c}\% \text { Boeing } \\
\text { Sample }\end{array}$ & $\begin{array}{c}\text { No. } \\
\text { Resps. }\end{array}$ & $\begin{array}{c}\text { \% DOE } \\
\text { Sample }\end{array}$ & $\begin{array}{c}\text { No. } \\
\text { Resps. }\end{array}$ & $\begin{array}{c}\text { \% Total } \\
\text { Sample }\end{array}$ \\
\hline Supervisor/Manager (SUP) & 151 & 21.7 & 31 & 24.2 & 187 & 21.3 \\
\hline $\begin{array}{l}\text { Non-Supervisor/Non-Man- } \\
\text { ager (NSUP) }\end{array}$ & 523 & 75.0 & 93 & 72.7 & 640 & 72.8 \\
\hline Unknown & 23 & 3.3 & 4 & 3.1 & 52 & 5.9 \\
\hline
\end{tabular}

Note: Numbers do not total overall due to individuals who indicated Supervisory Level but not organization. 
Table 3.4 presents information on the locations of the respondents, with the percent of the Boeing, the DOE, and the total SPR sample who classified themselves into each location. The majority of both DOE and Boeing employees who responded to the survey were at the New Orleans Location. The second highest percentage of respondents for both organizations indicated they were located at the Big Hill Location. The lowest percentage of Boeing respondents occurred at the Saint James Location while for DOE, the lowest percentage occurred at the Sulphur Mines Location.

Table 3.4. Distribution of Respondent Locations at SPR

\begin{tabular}{|l|c|c|c|c|c|c|}
\hline \multirow{2}{*}{ Location } & \multicolumn{2}{|c|}{ Boeing } & \multicolumn{2}{c|}{ DOE } & \multicolumn{2}{c|}{ Overall } \\
\cline { 2 - 7 } & $\begin{array}{c}\text { No. } \\
\text { Resps. }\end{array}$ & $\begin{array}{c}\text { \% Boeing } \\
\text { Sample }\end{array}$ & $\begin{array}{c}\text { No. } \\
\text { Resps. }\end{array}$ & $\begin{array}{c}\text { \% DOE } \\
\text { Sample }\end{array}$ & $\begin{array}{c}\text { No. } \\
\text { Resps. }\end{array}$ & $\begin{array}{c}\text { \% Total } \\
\text { Sample }\end{array}$ \\
\hline New Orleans (NO) & 348 & 49.9 & 70 & 54.7 & 438 & 49.8 \\
\hline Bayou Choctaw (BC) & 41 & 5.9 & 10 & 7.8 & 51 & 5.8 \\
\hline Bryan Mound (BM) & 60 & 8.6 & 9 & 7.0 & 71 & 8.1 \\
\hline Big Hill (BH) & 77 & 11.0 & 12 & 9.4 & 93 & 10.6 \\
\hline Weeks Island (WI) & 32 & 4.6 & 3 & 2.3 & 36 & 4.1 \\
\hline West Hackberry (WH) & 47 & 6.7 & 11 & 8.6 & 59 & 6.7 \\
\hline Sulphur Mines (SM) & 22 & 3.2 & 1 & 0.8 & 23 & 2.6 \\
\hline Saint James (SJ) & 18 & 2.6 & 2 & 1.6 & 21 & 24 \\
\hline Unknown & 52 & 7.5 & 10 & 7.8 & 87 & 9.9 \\
\hline
\end{tabular}

Table 3.5 presents the number of years employees of the Boeing, DOE and overall SPR o:ganizations indicated they had been employed at SPR. For the Boeing Organization, the largest percentage of respondents, 36.6 percent, indicaterl they had been employed at SPR between 4 and 6 years. For the DOE Organization, the highest percentage of respondents indicated they had been employed at SPR between 1 and 3 years (22.7 percent). For both Boeing and DOE, as well as for the overall organization, the lowest percentage of employces indicated they had been employed at SPR greater than 15 years $(.6,8$, and 1.1 percent respectively). Overall, the greatest percentage of employees indicated they had been employed at SPR between 4 and 6 years (33.0 percent).

Table 3.6 depicts the number of respondents for each educational level and the percentage that number represents for SPR. The greatest number of respondents at SPR had some college (22.0 percent).

Table 3.7 presents the modal educational level and mean number of years a! SPR for the survey respondents in each organization, as well as for the overall organization. For both the Boeing and the DOE Organizations, as well as for the overall SPR Organization, the modal educational level was Some College. The organization with the shortest tenure was Boeing, with an average length of time at SPR of 7.03 years. The DOE organization had the longest tenure at SPR, 8.18 years. The mean length of time of employment at SPR for the entire sample which took the survey was 7.29 years. 
Table 3.5. Number of Years at SPR

\begin{tabular}{||l|c|c|c|c|c|c||}
\hline \multirow{2}{*}{ Years at SPR } & \multicolumn{2}{c|}{ Boeing } & \multicolumn{2}{c|}{ DOE } & \multicolumn{2}{c|}{ Overall } \\
\cline { 2 - 7 } & $\begin{array}{c}\text { No. } \\
\text { Resps. }\end{array}$ & $\begin{array}{c}\text { \% Boeing } \\
\text { Sample }\end{array}$ & $\begin{array}{c}\text { No. } \\
\text { Resps. }\end{array}$ & $\begin{array}{c}\text { \% DOE } \\
\text { Sample }\end{array}$ & $\begin{array}{c}\text { No. } \\
\text { Resps. }\end{array}$ & $\begin{array}{c}\text { \% Total } \\
\text { Sample }\end{array}$ \\
\hline$<6$ months & 21 & 3.0 & 9 & 7.0 & 31 & 3.5 \\
\hline $1-3$ years & 92 & 13.3 & 29 & 22.7 & 125 & 14.2 \\
\hline $4-6$ years & 255 & 36.6 & 27 & 21.1 & 290 & 33.0 \\
\hline $7-9$ years & 156 & 22.4 & 18 & 14.1 & 181 & 20.6 \\
\hline $10-12$ years & 113 & 16.2 & 22 & 17.2 & 143 & 16.3 \\
\hline $13-15$ years & 33 & 4.7 & 15 & 11.7 & 194 & 22.1 \\
\hline$>15$ years & 4 & 0.6 & 1 & 0.8 & 10 & 1.1 \\
\hline Unknown & 24 & 3.4 & 7 & 5.5 & 49 & 5.6 \\
\hline
\end{tabular}

Note: Numbers do not total overall due to people who indicated number of years but not organization.

Table 3.6. Educational Level of SPR Respondents

\begin{tabular}{||l|r|r|}
\hline \multicolumn{1}{|c|}{ Educational Level } & No. Responses & \% Total Sample \\
\hline Some High School & 17 & 1.9 \\
\hline High School Degree & 132 & 15.0 \\
\hline Some Technical School & 74 & 8.4 \\
\hline 2-Year Technical Degree & 31 & 3.5 \\
\hline Some College & 193 & 22.0 \\
\hline 2-Year College Degree & 51 & 5.8 \\
\hline 4-Year College Degree & 140 & 15.9 \\
\hline Some Graduate Work & 74 & 8.4 \\
\hline Graduate Degree & 102 & 11.6 \\
\hline Unknown & 65 & 7.4 \\
\hline
\end{tabular}


Table 3.7. Modal Educational Level and Mean Number of Years at SPR Overall and for Each Organization

\begin{tabular}{|l|c|r|}
\hline \multicolumn{1}{|c|}{ Organization } & Educational Level' $^{\prime}$ & Years at SPR \\
\hline Overall & 5 & 7.29 \\
\hline Boeing & 5 & 7.03 \\
\hline DOE & 5 & 8.18 \\
\hline
\end{tabular}

${ }^{1}$ For Educational Level, 5 = Some College. 


\section{ORGANIZATIONAI. SURVEY SCALES AND RESULTS}

The Organizational Survey (OS) administered at the Strategic Petroleum Reserve (SPR) was comprised of the Organizational Culture Inventory (OCI) (Human Synergistics, 1987), which consists of 12 scales, and scales which assess communication processes, commitment to the organization, cohesiveness of work group, coordination of work, overall job satisfaction, perceived hazardous nature of work, attention to safety, and questions concerning environniental issues. The results from each of these scales are discussed in the sections that follow. Sections present the overall results for SPR on the scales, the results by organization, by location, by department, within organization, by employee category, both overall and within organization, and by supervisory level, both overall and within organization.

\subsection{Organizational Survey Scale Descriptions}

\subsubsection{Organizational Culture Inventory}

The philosophy of management, the mission of the organization, and the strategic choices management makes determine the culture of the organization (Cooke and Burack, 1987). The aspect of culture most immediately affected by these factors is what is valued by the organization. The extent to which these values are recognized and shared reflects the strength of the organization's culture. Organizational factors, along with these shared values, influence the operating structures of the organization, it's human resource management practices, and the styles of it's supervisors. To the extent that these shared values and behavioral norms can be measured and evaluated, data collection of this type is importani in understanding the organizational factors that influence performance.

The Organizational Culture Inventory (OCI) (Human Synergistics, 1987) is a paper-and-pencil diagnostic system for measuring the aspects of organizational culture that have the greatest impact on the activities of members and the functioning of the organization. Respondents are asked to review 120 statements which describe some of the thinking and behavioral styles that members of an organization may be expected to adopt in carrying out their work and in interacting with others. These statements comprise 12 different cultural scales, some of which are indicative of a positive and supportive environment, while others are useful in identifying potentially dysfunctional environments. All of the scales measured by the $\mathrm{OCI}$ are related to, and result from, organizational structural variables, reward systems, managerial styles and philosophies, and other factors that can be changed, at least to some extent, by those in leadership positions.

The 12 organizational culture scales, with examples of the items used to assess each one, are described below. For a complete listing of the OCI scalc items, see Human Synergistics (1987).

C1: HUMANISTIC-ENCOURAGING: Organizations which are managed in a participative and personcentered way. Members are expected to be supportive, constructive, and open to influence in their dealings with one another.

- Involving subordinates in decisions;

- Showing concern for the needs of others.

C2: AFFILIATIVE: Organizations which place a high priority on constructive personal relations. The members are expected to be friendly, open, and sensitive to the satis'action of their work group.

- Thinking in terms of the group's satisfaction; 
- Using good human relations skills.

C3: APPROVAL: Organizations in which conflicts are avoided and personal relations are pleasant, at least superficially. Members feel they should agree with and gain approval of others.

- Staying on the good side of superiors;

- Making sure people accept you.

C4: CONVENTIONAL: Organizations that are conservative, traditional, and bureaucratically controlled. Members are expected to conform, follow rules, and make a good impression.

- Always following policies and practices;

- Avoiding confrontations.

C5: DEPENDENT: Organizations that are hierarchically controlled and non-participative. Centralized decision making leads members to do only what they are told and to clear all decisions with superiors.

- Accepting goals without questioning them;

- Never challenging superiors.

C6: AVOIDANCE: Organizations that do not reward success but punish failure. Negative rewards lead members to shift responsibility to others and avoid being blamed for mistakes.

- Taking few chances;

- Laying "low" when things get tough.

C7: OPPOSITIONAL: Organizations in which confrontation prevails and negativism is rewarded. Members gain status and influence by being critical and are encouraged to oppose the ideas of others.

- Pointing out flaws;

- Remaining aloof from the situation.

C8: POWER: Non-participative organizations which are structured on the basis of authority in members' positions. Members expect to take charge, control subordinates, and respond to demands of superiors.

- Demanding loyalty;

- Acting forceful.

C9: COMPETITION: Organizations where winning is valued and rewards are given for out-performing others. Members operate in a "win-lose" framework and work against their peers to be noticed.

- Always trying to be right;

- Out-performing one's peers.

C10: PERFECTIONISTIC: Organizations in which persistence, hard work, and perfectionism are highly valued. Members feel they must avoid all mistakes, keep track of everything, and work long hours to attain specific objectives.

- Setting unrealistically high goals; 
- Viewing work as more important than anything else.

C11: ACHIEVEMENT: Organizations that do things well and value members who set and accomplish their own goals. Members set challenging, but realistic goals, and plan and pursue them with enthusiasm.

- Exploring alternatives before acting;

- Pursuing a standard of excellence.

C12: SELF-ACTUALIZING: Organizations that value creativity, quality over quantity, tasks, and individual growth. Members are encouraged to gain satisfaction from their work, develop themselves, and take on new activities.

- Thinking in unique and independent ways;

- Communicating ideas.

From these twelve scales, three cultural styles are described. The first style is comprised of the Humanistic-Encouraging (C1), Affiliative (C2), Achievement (C11), and Self-Actualizing (C12) Scales. Thesc scales are considered "Constructive Styles;" in other words, organizations which score high on these four scales tend to promote behaviors which are conducive to the satisfaction of the organizational members.

The second cultural style is the "Passive/Defensive Style." This style is made up of the Approval (C3), Conventional (C4), Dependent (C5), and Avoidance (C6) Scales. In organizations which score high on these scales, a culture exists which leads employees of the organization to act and react in a defensive way and at the same time, act in a way which does not pose a threat to one's own security within that organization.

A third cultural style is made up of the Oppositional (C7), Power (C8), Competitive (C9), and Perfectionistic (C10) Scales. Organizations which score high on these scales often expect members to act in a way that is forceful and that protects one's position and status. In other words, members adopt an "Aggressive/Defensive Style" in order to be successful within the organization.

\subsubsection{Communication Scales}

Communication is a critical process for effective operations in any organization. However, because it is a process rather than a variable, it is very difficult to measure. The scales used in the questionnaire administered at SPR were developed by Roberts and O'Reilly (1974). They have been administered to various organizations with good reliability and success in analyzing several facets of the communication process.

Four communication scales were administered and are described below. The range on each scale is from a low score of 1 to a high score of 7 .

TRUST: $\quad$ Freedom to discuss the problems and difficulties in the job with an immediate supervisor without jeopardy.

ACCURACY: Perception of the accuracy of information received from other organizational levels (superior, same, and subordinate levels). 
INTERACT: Desirability of frequent contact with others in the organization (superiors, same, and subordinates).

SATISFAC: Overall satisfaction with the communication process in the organization.

\subsubsection{Commitment Scale}

The Commitment Scale is defined as the relative strength of an individual's identification with and involvement in a particular organization (Mowday \& Steers, 1979). This commitment extends to the goals of the organization and the desire to maintain membership in the organization to facilitate these goals. The range on this scale is from a low score of 1 (low commitment) to a high score of 7 (high commitment).

\subsubsection{Cohesion Scale}

The Cohesion Scale is very similar to the Commitment Scale except that it is delined as the relative strength of an individual's identification with and involvement in a particular work group (Seashore, 1954; Price \& Muller, 1972). The range on this scale is from a low score of 1 (weak cohesiveness) to a high score of 7 (strong cohesiveness).

\subsubsection{Coordination Scale}

The Coordination Scale assesses the employee's perception of the degree to which the subunits of an organization operate according to the requirements of each other and of the total organization (Georgopoulos \& Mann, 1962). The range on this scale is from a low score of 1 (low coordination) to a high score of 7 (high coordination).

\subsubsection{Job Satisfaction}

The Job Satisfaction Scale (Kunin, 1955) refers to employees' overall satisfaction with their jobs. While it is not able to point to specific aspects of the working environment which people are satisfied or dissatisfied with, it can help in determining whether employee satisfaction is something which needs further consideration by management. The scale ranges from a low score of 1 (very dissatisfied) to a high score of 7 (very satisfied).

\subsubsection{Hazard Scale}

The Hazard Scale is used to identify people's perception of the hazardous nature of their work (K.H. Roberts, 1990, personal communication). The scale ranges from a low score of 1 (not hazardous) to a high score of 7 (very hazardous).

\subsubsection{Safety Scale}

The Safety Scale, developed by researchers at the University of California at Berkeley (K. II. Roberts, 1989, personal communication), is used to assess an individual's perception of the importance of safety to success in an organization. Safety is defined as operating in a manner to ensure that the probability of making a mistake is low, because the consequence of making a mistake is high. Organizations typically viewed as operating in this manner are nuclear reactors, naval aircraft carriers and 
air traffic control centers. The safety scale consists of 40 items which range from a low score of 1 (does not help at all) to a high score of 7 (helps a great deal).

\subsubsection{Environment, Safety, and Health Questions}

For the administration of the Organizational Survey (OS) at SPR, four questions pertaining to environment, safety, and health (ES\&H) issues were used. Each question ranges from a low score of 1 (not at all or little) to a high score of 7 (very likely or a lot).

The first ES\&H question deals with the likelihood of serious offsite environmental damages/consequences due to improper or substandard performance uy a work group. The second ES\&H question deals with the likelihood of serious onsite environmental damages/consequences due to improper or substandard performance by a work group. The third ES\&H question asks employees to assess the amount of emphasis they believe management places on environmental issues. Finally, the fourth ES\&H question asks employees for their perception of how well informed they are of possible risks in their work environment.

\subsection{Overall Results on the OS Scales for SPR}

\subsubsection{Organizational Culture Inventory Results}

The overall mean scores on the OCI Scales for the entire sample of SPR employees who responded to the Organizational Survey (OS) are depicted in Figure 4.1. The scales are identified by number and are described in the preceding section. The scores represent the mean score for the entire sample where the score 1 equals not at all and the score 5 equals to a great extent.

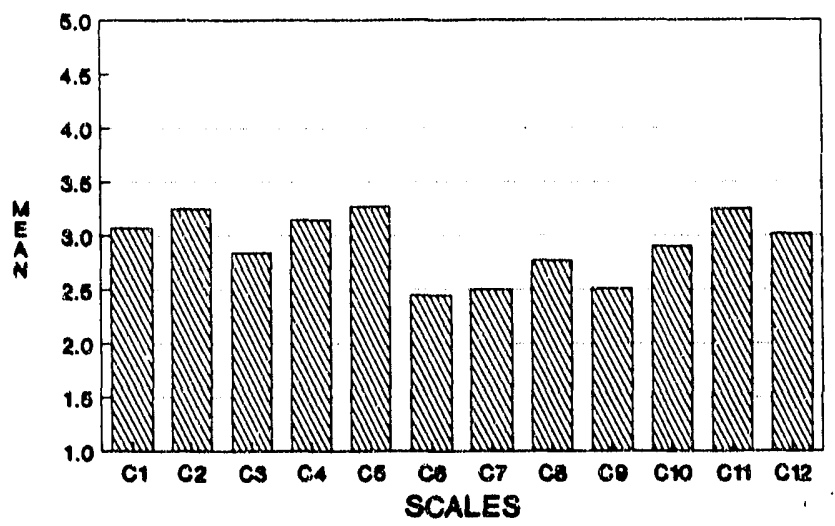

Figure 4.1. Overall mean values on the OCI scales for SPR

As seen in Figure 4.1, the SPR respondents had the highest mean scores on those scales which relate to the Passive - Defensive Cultural Style, particularly the Approval (C3), Conventional (C4), and Dependent (C5) Scales. Across all twelve scales, the highest mean value was found on the Dependent (C5) Scale. Additionally, the Conventional (C4) Scale had an obtained mean value that was higher than two of the four scales in the Constructive Cultural Style. This suggests that the predominant cultural style which exists at SPR is passive - defensive. Thus, SPR employees particularly perceive expectations of avoidance, conformity, dependence, and a need for the approval of others. The values obtained on 
the scales which comprise the Constructive Cultural Style (i.e. Humanistic - Encouraging (C1), Affiliative (C2), Achievement (C11), and Self-Actualizing (C12) Scales) tended to be high as well, although this is routinely found at most facilities surveyed. Thus despite the generally higher mean values obtained on the Constructive Cultural Style Scale, the emphasis is placed on the Passive Defensive Cultural Style. The mean values obtained on the Aggressive -Defensive Cultural Style were also relatively high, particularly on the Power (C8) Scale.

\subsubsection{Communication Scales Results}

Figure 4.2 depicts the overall mean values obtained for the SPR sample on the four communication scales. The survey respondents soored higher on the Perceived Accuracy of Communication Scale than on the Trust in Communication Scale, although the mean value obtained for both was relatively low. The sample of SPR employees had a moderately high desire for interaction and communication with others in their organization, as represented by the mean score on the Desire for Interaction - Communication Scale. The SPR employees had only a moderate amount of satisfaction with the communication processes at SPR as described by the Comnunication - Satisfaction Scale.

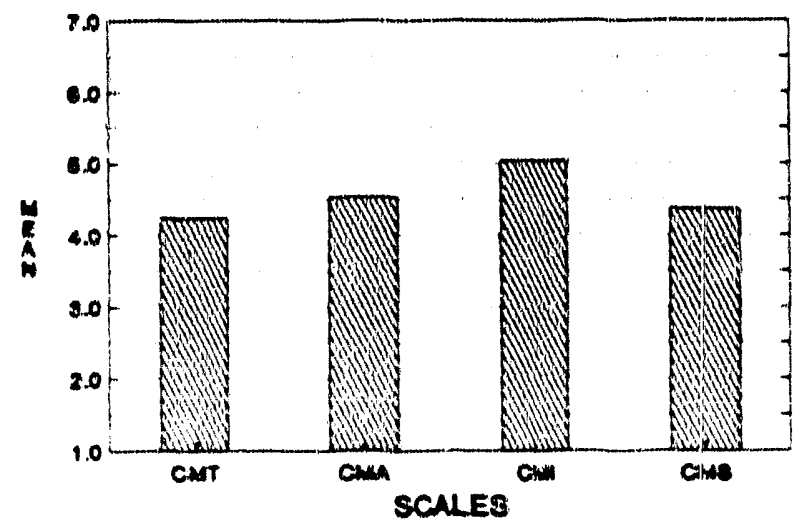

Figure 4.2. Overall mean values on communication scales for SPR

\subsubsection{Resulis for Additional Scales}

Figute 4.3 presents the results obtained on the Commitment, Cohesion, Hazard, Safety, Coordination, and Job Satisfaction Scales for the SPR sample. Riespondents indicated a moderate amount of commitment to their organization, and a higher amount of cohesion within their own working groups. On average, SPR respondents perceived there to be a low amount of hazard associated with their work. This is seen in the mean value obtained on the Hazard Scale which is below the midpoint of 4.0. However, respondents did indicate that safety related attributies are helpful to them in doing their job well. The perception of coordination among working groups was low, evidenced by a mean value below the scale midpoint value of 4 . SPR respondents also indicated a moderately high amount of satisfaction with their jobs.

\subsubsection{Environment, Safety, and Health Questions Results}

Respondents from the SPR sample perceived the potential for both onsite and offsite environmental consequences from poor or substandard work performance to be low (Figure 4.4). The 
perception for onsite consequences was slightly higher than that for offsite consequences. SPR respondents indicated that management places a moderately high amount of emphasis on environmental issues and that employees who work at SPR are fairly well aware of potential risks associated with their jobs. The respondents indicated a slightly lower amount of employee awareness than management emphasis on environment, safety, and health issues relevant to SPR.

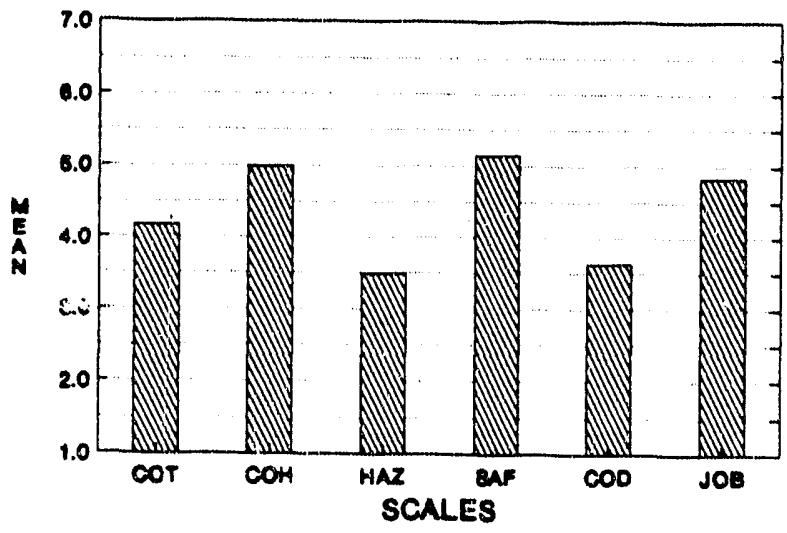

Figure 4.3. Overall mean values on additional scales for SPR

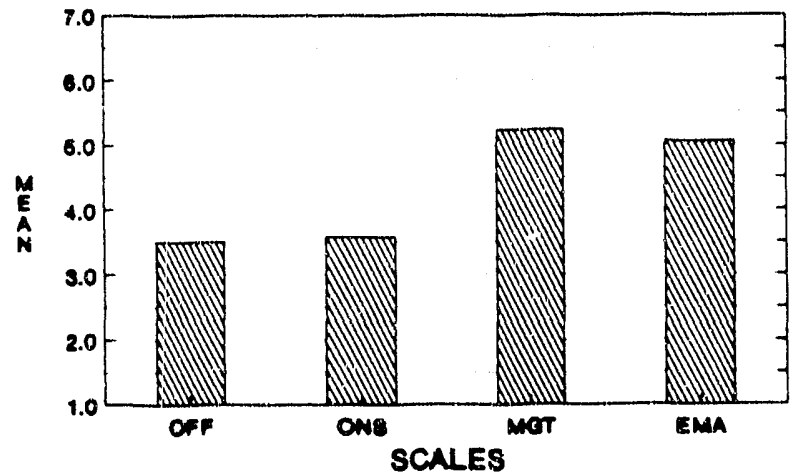

Figure 4.4. Overall mean values on environment, safety, and health questions for SPR

\subsubsection{Summary}

The overall profile of SPF indicates an organization that has a strong tendency to deal with issues in a passive-defensive manner, although there is also an expectation that work should be completed in a constructive and creative manner. The issue of defensiveness is further supported by the somewhat low values on the Communication - Trust and Accuracy Scales. The perception also exists that working units are not well coordinated, although within working groups there does appear to be a relatively high amount of cohesion. There is the perception that a low amount of hazard as well as environmental consequences exists at SPR. Additionally, employees believe that those attributes important to safety are helpful to them in doing their jobs well. Finally, respondents' perception of management emphasis on, and employee awareness of environmental issues are moderately high. 


\subsection{Differences Between Organizations on the OS Scales}

\subsubsection{Differences Between Organizations on the $\mathrm{OCI}$}

No statistically significant differences between the Boeing and DOE Organizations at SPR were found on any of the OCI Scales. Appendix $A$ presents the mean values obtained for each SPR Organization on each of the scales on the OS. Appendix B contains figures which compare the mean value obtained for the overall SPR Organization, to the mean values obtained for the Boeing and DOE Organizations on the OCI Scales.

\subsubsection{Differences Between Organizations on the Communication Scales}

No statistically significant differences were obtained between the Boeing and DOE Organizations at SPR on any of the Communication Scales. Appendix A presents the mean values obtained for each SPR Organization on each of the scales on the OS. Appendix $C$ contains figures which compare the mean value obtained for SPR overall to the mean values obtained for both Boeing and DOE on each of the Communication Scales.

\subsubsection{Differences Between Organizations on the Additional Scales}

No statistically significant differences occurred between the Boeing and DOE Organizations on any of the auditional scales. The mean values obtained for each organization on each of the additional scales are contained in Appendix A. Appendix $D$ contains figures which compare the mean values obtained for the overall SPR Organization to the mean values obtained by both Boeing and DOE on each of the additional scales.

\subsubsection{Differences Between Organizations on the Environment, Safety, and Health Questions}

No statistically significant differences between the Boeing and DOE Organizations at SPR were obtained on any of the Environment, Safety, and Health Questions. The mean values obtained for each department on the questions are contained in Appendix A. Also, Appendix E presents figures which compare the mean values obtained by the overall SPR Organization to the mean values obtained by the Boeing and DOE Organizations on the Environment, Safety, and Health Questions.

\subsubsection{Summary}

No statistically significant differences were obtained between the Boeing and DOE Organizations at SPR on any of the scales contained in the OS. This suggests that the conclusions discussed for the overall organization at SPR (Section 4.2.5) are appropriate for both the Boeing and DOE Organizations. This lack of statistically significant differences between the two organizations is somewhat surprising given the different functional roles these organizations fill at SPR.

\subsection{Differences Between Locations on the OS Scales}

\subsubsection{Differences Between Locations on the OCI Scales}

No statistically significant differences were obtained between the SPR Locations on any of the OCI Scales. Appendix F contains the mean values obtained on each scale of the OS for each of the SPR Locations. 


\subsubsection{Differences Between Locations on the Communication Scales}

No statistically significant differences were obtained between the SPR Locations on any of the Communication Scales. Appendix F presents the mean values obtained for each of the locations on each of these scales.

\subsubsection{Differences Between Locations on the Additional Scales}

Statistically significant differences between the SPR Locations were obtained on four of the additional scales: Cohesion, Hazard, Safety, and Coordination. The mean values obtained by each of the locations on all of the additional scales are presented in Appendix F.

Statistically significant differences obtained between the locations on the Cohesion Scale are presented in Figure 4.5. The Sulphur Mines had the highest mean value on this scale and was statistically significantly different from New Orleans, West Hackberry, and Bryan Mound. Bryan Mound had the lowest mean value on this scale and was also statistically significantly different from the Saint James Location.

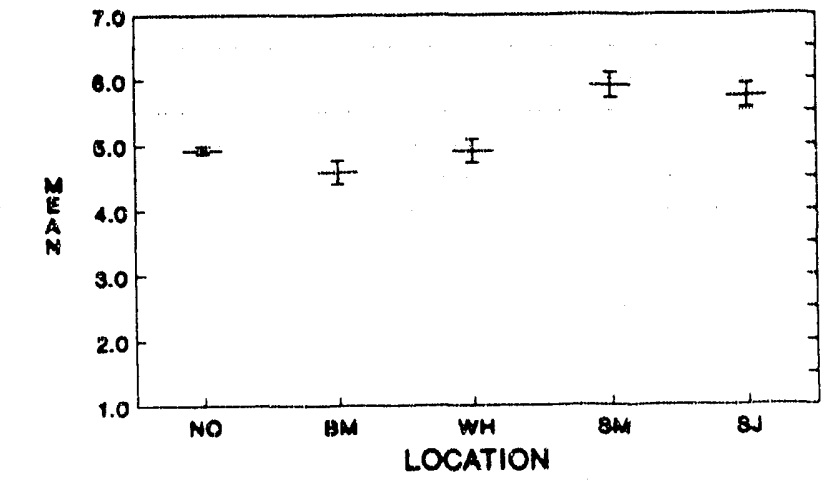

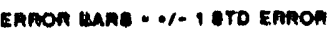

Figure 4.5. Significant differences between SPR locations on the cohesion scale

Statistically significant differences between locations on the Hazard Scale are presented in Figure 4.6. The New Orleans Location had the lowest mean value on this scale and was statistically significantly different from every other SPR Location. The Weeks Island Location had the highest mean value on this scale.

Statistically significant differences between SPR Locations were also obtained on the Safety Scale. Figure 4.7 depicts the statistically significant differences obtained. The New Orleans Location had the lowest mean value on this scale and was statistically significantly different from the Big Hill, Weeks Island, and Sulphur Mines Locations. The Sulphur Mines Location had the highest mean value on this scale.

Statistically significant differences between the SPR Locations on the Coordination Scale a a presented in Figure 4.8. The Suipnui Mines had the highest mean value on this scale and was statistically significantly different from the Weeks Island, New Orleans, and Bryan Mound Locations. The Bryan Mound Location had the lowest mean value on this scale and was also statistically significantly different from the Bayou Choctaw, Big Hill, and Saint James Locations. 


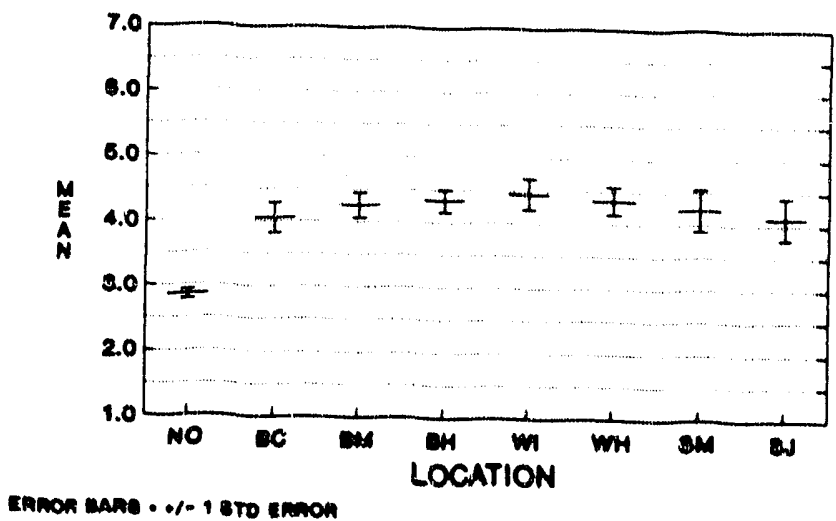

Figure 4.6. Significant differences between SPR locations on the hazard scale

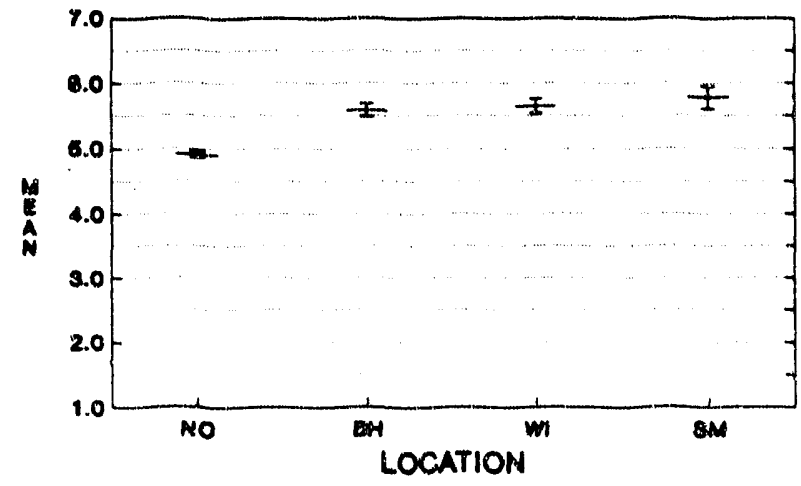

Ehnon aAme - of- 1 gto Enmon

Figure 4.7. Significant differences between SPR locations on the safety scale

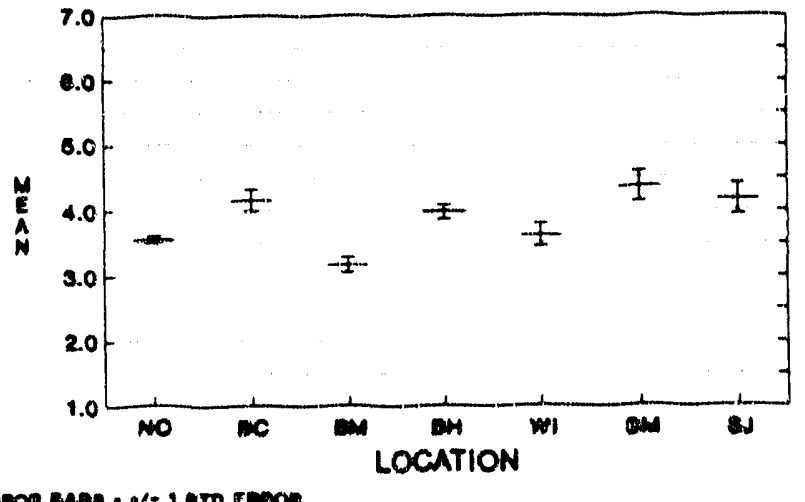

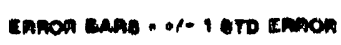

Figure 4.8. Significant differences between SPR locations on the coordination scale 


\subsubsection{Differences Between Locations on the Environment, Safety, and Health Questions}

Statistically significant differences were obtained between the SPR Locations on all four of the Environment, Safety, and Health Questions. The mean values for each location on each of the questions are also contained in Appendix F.

Figure 4.9 presents the statistically significant differences obtained between the SPR Locations on the Offsite Environmental Consequences Question. New Orleans had the lowest mean value on this question and was statistically significantly different from the Bayou Choctaw, Big Hill, Weeks Island, and West Hackberry Locations. The Weeks Island Location had the highest mean value on this question.

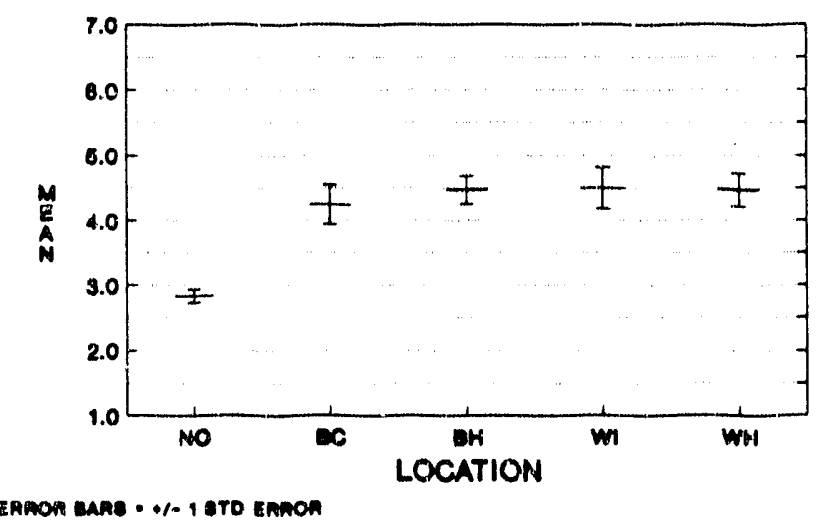

Figure 4.9. Significant differences between SPR locations on the offsite consequences question

On the Onsite Environmental Consequences Question (Figure 4.10), the New Orleans Location had the lowest mean value and was statistically significantly different from every other location. The Weeks Island Location had the highest mean value on this scale of all the SPR locations.

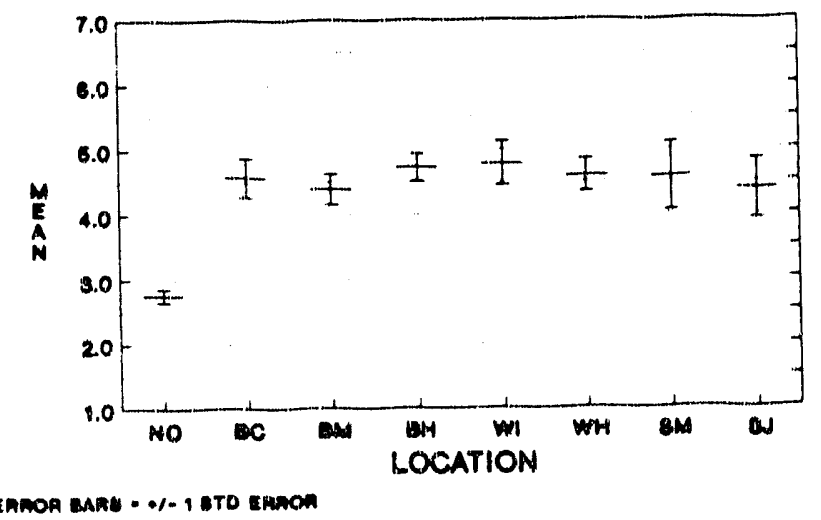

Figure 4.10. Significant differences between SPR locations on the onsite consequences question 
Figure 4.11 presents the statistically significant differences obtained between the SPR Locations on the Management Emphasis Question. The New Orleans Location had the lowest mean value on this question and was statistically significantly different from every other location except the Bryan Mound Location. The Saint James Location had the highest mean value on this question.

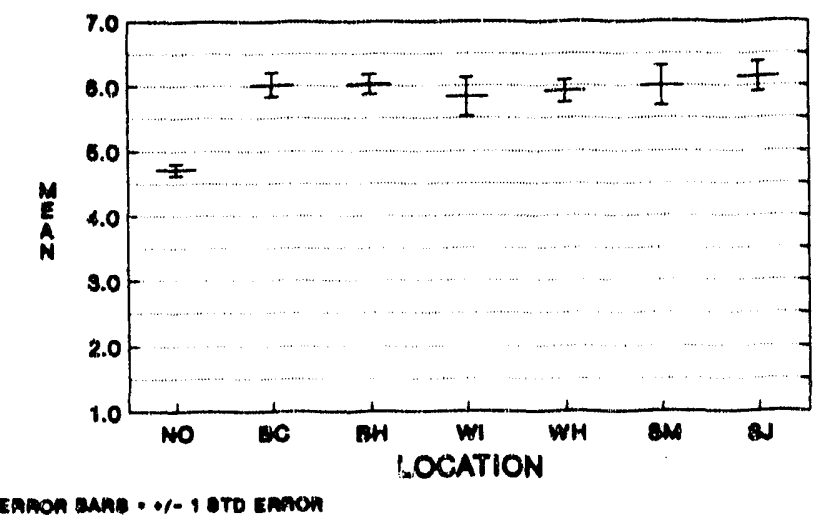

Figure 4.11. Significant differences between SPR locations on the management emphasis question

Statistically significant differences between the SPR Locations obtained on the Employee Awareness Question are depicted in Figure 4.12. The New Orleans Location had the lowest mean value on this question and was statistically significantly different from the Big Hill, Sulphur Mines, and Saint James Locations. The Sulphur Mines Location had the highest mean value on this question.

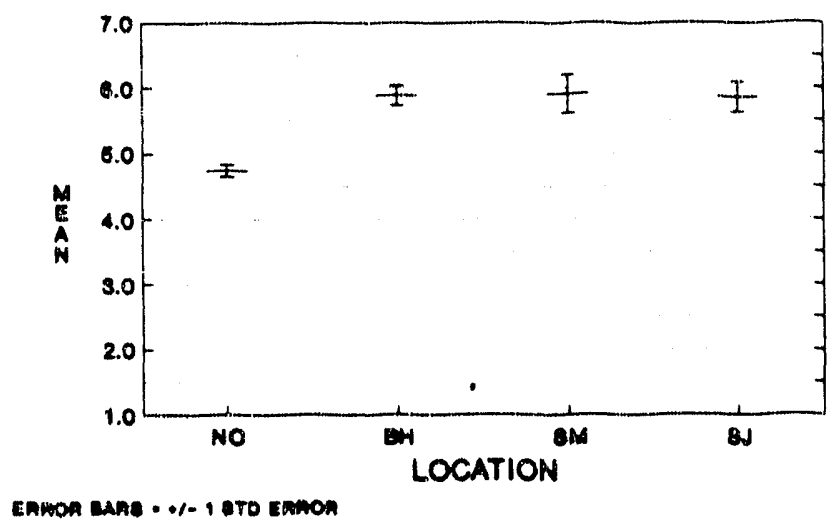

Figure 4.12. Significant differences between SPR locations on the employee awareness question

\subsubsection{Summary}

Statistically significant differences obtained between the SPR Locations were obtained only on the additional scales and on the Environment, Safety, and Health Questions. No statistically significant differences were obtained on the $\mathrm{OCI}$ or Communication Scales. This suggests that the issues raised for the overall organization in terms of its organizational culture and communication processes are common across all SPR Locations. On the other scales, the New Orleans Location is the consistent outlier from 
the other SPR Locations, especially on those scales relating to the hazardousness or environmentally consequential nature of work. It is are lower on the Cohesion, Hazard, Safety, and Coordination Scales as well as on the Offsite Consequences, Onsite Consequences, Management Emphasis, and Employee Awareness Questions. While the other locations do appear to group similarly on these scales, there are some differences that exist there as well. For example, the Bryan Mound Location is lower than other locations on both the Cohesion and Coordination Scales. In addition, it is the only location which does not differ from the New Orleans Location on the Management Emphasis Question. The Weeks Island Location tends to be lower than some of the other locations on the Coordination Scale, while the West Hackberry Location tends to be lower than other locations on the Cohesion Scale. The Sulphur Mines Location least resembles the New Orleans Location across most of the scales.

\subsection{Differences Between Employee Categories on the OS Scales}

\subsubsection{Differences Between Employee Categories on the OCI Scales}

No statistically significant differences were obtained between the SPR Employee Categories on the $\mathrm{OCl}$ Scales. Appendix $G$ presents the mean values obtained for each category on all of the $O S$ Scales.

\subsubsection{Differences Between Employee Categories on the Communication Scales}

Statistically significant differences were obtained between employee categories on one of the Communication Scales: Communication Interaction. Appendix $G$ contains the mean values obtained for each employee category on each of the Communication Scales.

Figure 4.13 depicts the statistically significant differences obtained between the SPR Employee Categories on the Communication - Interaction Scale. The Non-Exempt Technical DOE employees had a statistically significantly higher mean value on this scale than the Hourly Boeing employees. No other statistically significant differences were obtained between employee categories on this scalc. .

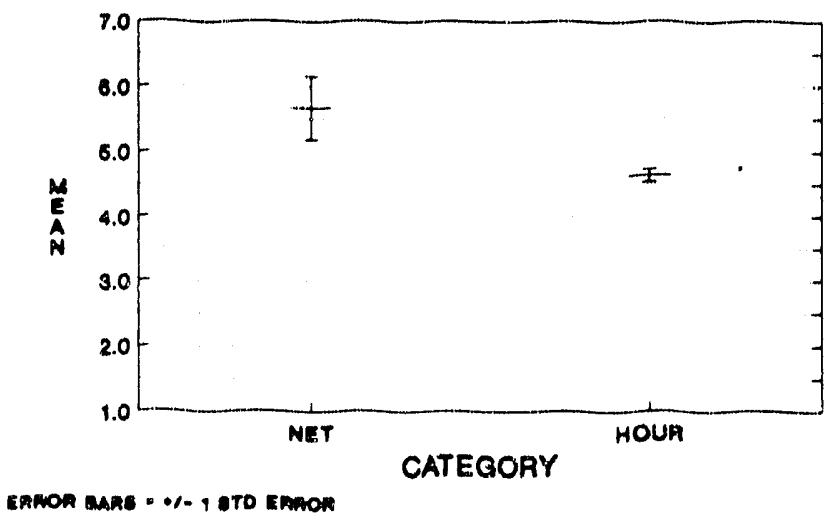

Figure 4.13. Significant differences between SPR employee categories on the communication-interaction scale 


\subsubsection{Differences Between Employee Categories on the Additional Scales}

Statistically significant differences were obtained between employee categories on one of the additional scales: Hazard. Appendix $G$ contains the mean values obtained for all employee categories on each of the scales contained in the OS.

Statistically significant differences between employee categories on the Hazard Scale are presented in Figure 4.14. The Hourly Boeing employees had a statistically significantly higher mean value on this scale than the Non-Exempt Boeing and the Non-Exempt Administrative DOE employees. The Non-Exempt Administrative DOE employees had the lowest mean value on this scale.

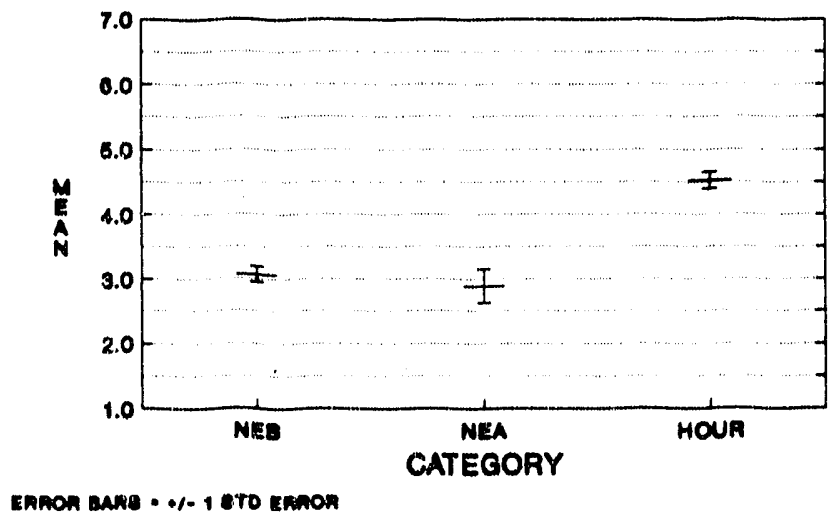

Figure 4.14. Significant differences between SPR employee categories on the hazard scale

\subsubsection{Differences Between Employee Categories on the Environment, Safety, and Health Questions}

Statistically significant differences were obtained between employee categories on all of the Environment, Safety, and Health Questions. In addition, Appendix G contains the mean values obtained for each category on each of these questions.

Figure 4.15 depicts the statistically significant differences obtained between employee categories on the Offsite Consequences Question. The Hourly Boeing employees had the highest mean value on this question and were statistically significantly different from the Non-Exempt Administrative DOE employees.

Figure 4.16 presents the statistically significant differences obtained between employee categories on the Onsite Consequences Question. The Hourly Boeing employees once again had the highest mean value on this question and were statistically significantly different from the Non-Exempt Administrative DOE employees.

Statistically significant differences between employee categories on the Management Emphasis Question are presented in Figure 4.17. The Hourly Boeing employees had the highest mean value on this question and were statistically significantly different from the Non-Exempt Administrative DOE employees. 


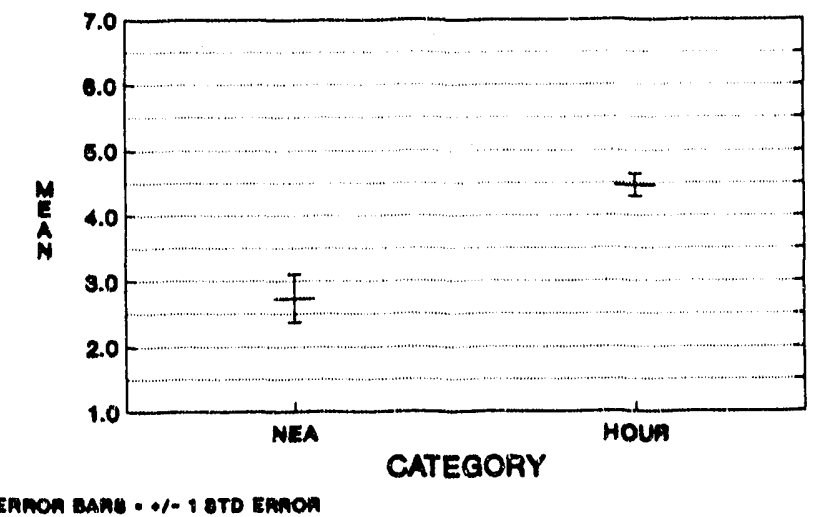

Figure 4.15. Significant differences between SPR employee categories on the offsite consequences question

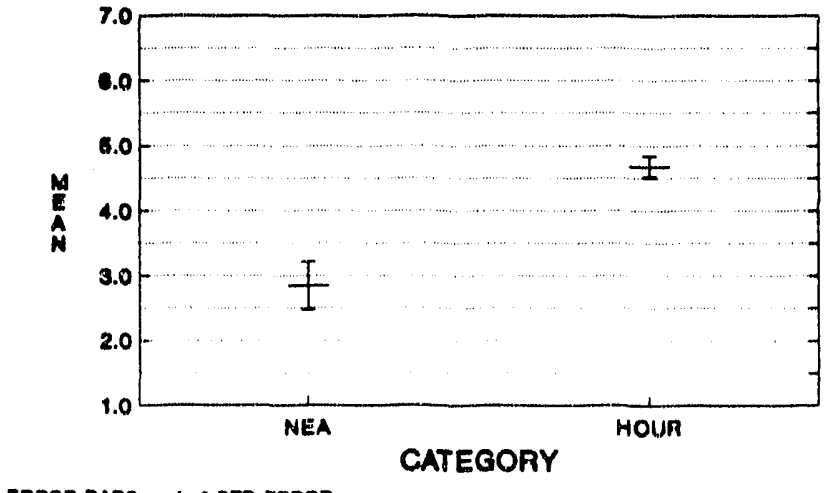

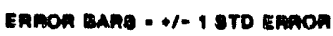

Figure 4.16. Significant differences between SPR employee categories on the onsite consequences question

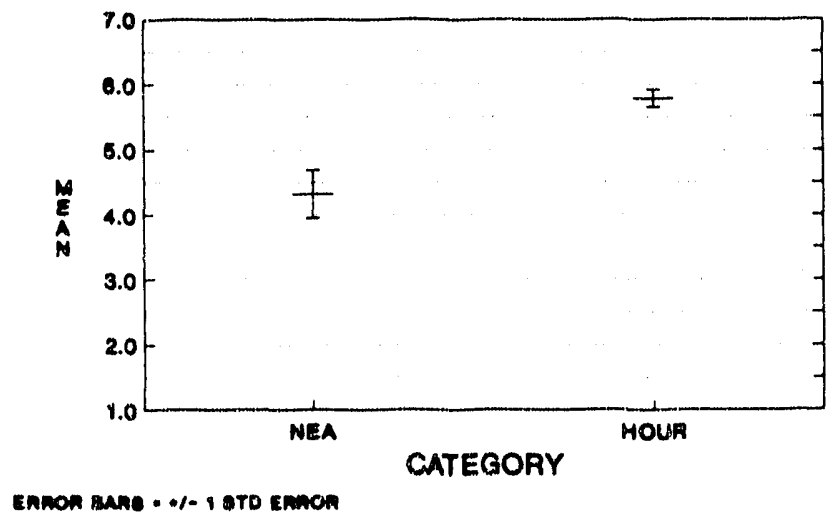

Figure 4.17. Significant differences between SPR employee categories on the management emphasis question 
Figure 4.18 presents the statistically significant differences obtained between the SPR Employee Categories on the Employee Awareness Question. The results obtained here are similar to those for the prior three Environment, Safety, and Health Questions. The Hourly Boeing Employee Category had the highest obtained mean value on this question and was statistically significantly different from the NonExempt Administrative DOE Employee Category.

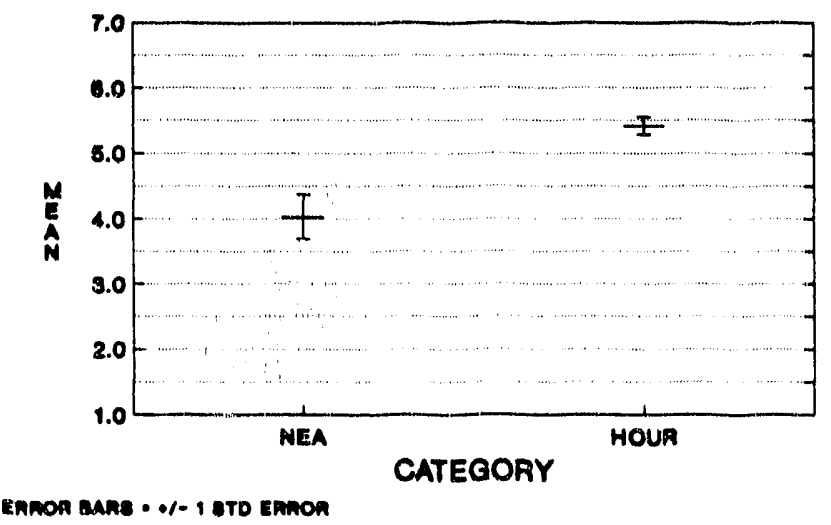

Figure 4.18. Significant differences between SPR employee categories on the employee awareness question

\subsubsection{Summary}

Few statistically significant differences were obtained between employee categories at SPR on the OS Scales. All differences, but one (Communication - Interaction), were on the scales/questions which are related to the hazardous aspects associated with one's job. In particular, the Hourly Boeing Employee Category had the perception that their jobs involve a significantly higher amount of hazard/environmental consequences than the Non-Exempt Administrative DOE Employee Category perceived. The Hourly Employee Category also perceived there to be a greater amount of management emphasis on and employee awareness of those environment and safety issues which are relevant to their jobs than the Non-Exempt Administrative DOE employees perceive.

\subsection{Differences Between Supervisory Levels on the OS Scales}

\subsubsection{Differences Between Supervisory Levels on the OCI Scales}

Statistically significant differences between the SPR Supervisory Levels on the HumanisticEncouraging (C1) Scale are presented in Figure 4.19. The Supervisors had a statistically significantly higher mean value on this scale than the Non-Supervisors.

Figure 4.20 depicts the statistically significant differences obtained between the SPR Supervisory Levels on the Affiliative (C2) Scale. The Supervisors had a statistically significantly higher mean value on this scale than the Non-Supervisors.

No statistically significant differences were obtained between the SPR Supervisory Levels on the Approval (C3) Scale. Appendix $G$ contains the mean values obtained for each level on this scale, as well as on all other OS Scales. 


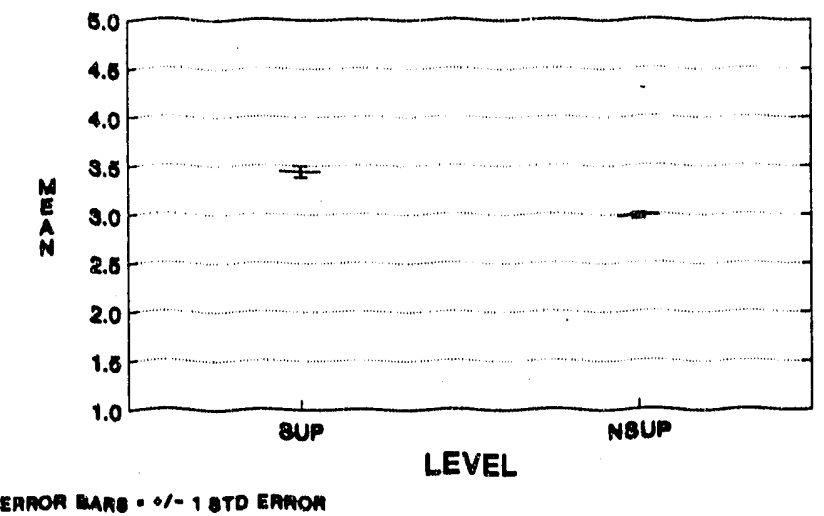

Figure 4.19. Significant differences between SPR supervisory levels on the humanistic-encouraging scale

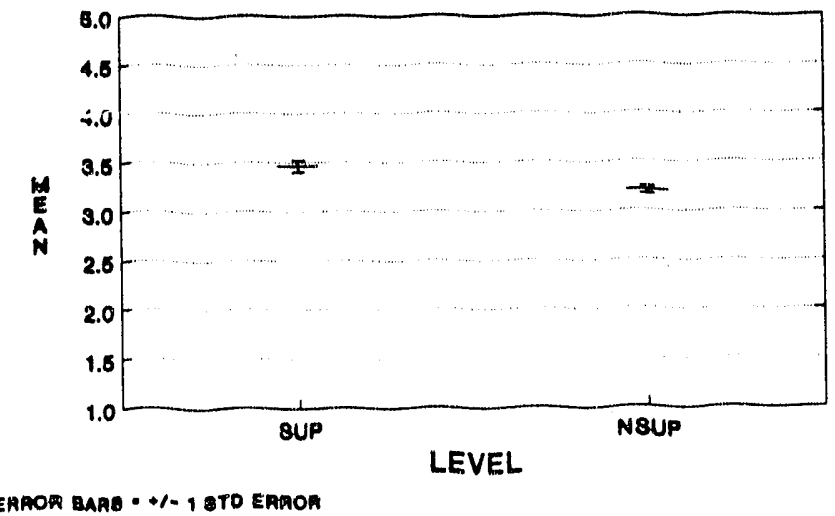

Figure 4.20. Significant differences between SPR supervisory levels on the affiliative scale

Figure 4.21 presents the statistically significant differences obtained between the SPR Supervisory Levels on the Conventional (C4) Scale. The Non-Supervisors had a statistically significantly higher mean value on this scale than the Supervisors.

No statistically significant differences were obtained between the SPR Supervisory Levels on the Dependent (C5), Avoidance (C6), Oppositional (C7), Power (C8), Competition (C9), and Perfectionistic (C10) Scales. Appendix $\mathrm{H}$ contains the mean values obtained by each level on each of these scales.

Figure 4.22 presents the statistically significant differences obtained between the SPR Supervisory Levels on the Achievement (C11) Scale. The Supervisors had a statistically significantly higher mean value on this scale than the Non-Supervisors.

Statistically significant differences obtained between the supervisory levels on the Self-Actualizing (C12) Scale are presented in Figure 4.23. The Supervisors had a statistically significantly higher mean value on this scale than the Non-Supervisors. 


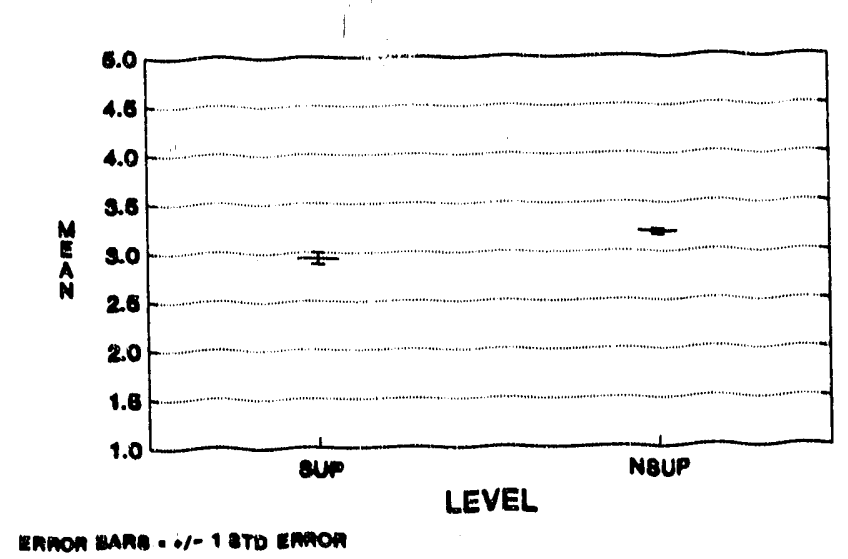

Figure 4.21. Significant differences between SPR supervisory levels on the conventional scale

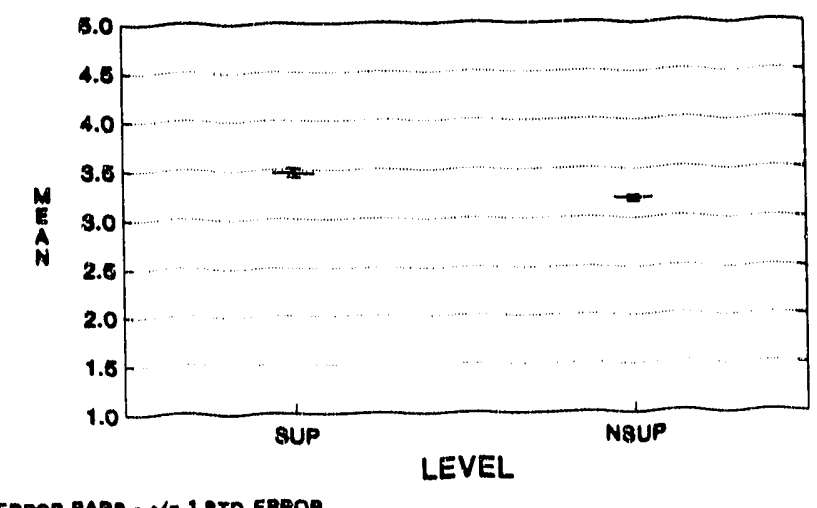

Figure 4.22. Significant differences between SPR supervisory levels on the achievement scale

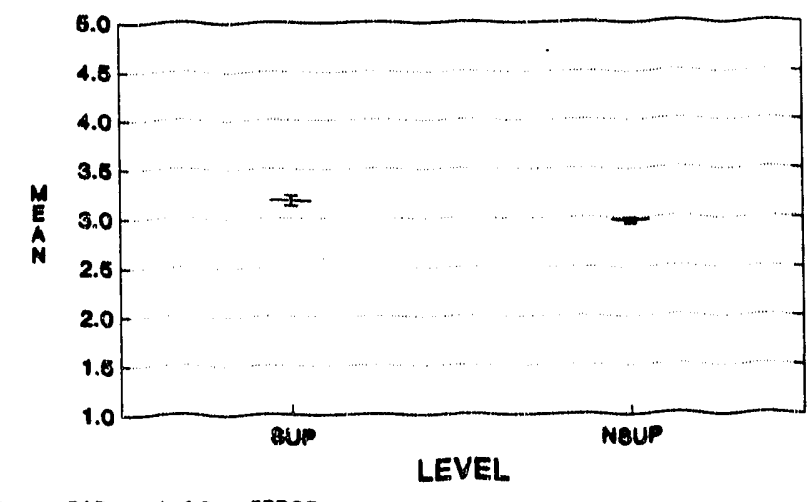

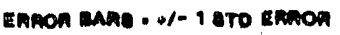

Figure 4.23. Significant differences between SPR supervisory levels on the self-actualizing scale 


\subsubsection{Differences Between Superisory Levels on the Communication Scales}

Statistically significant differences between supervisory levels were obtained on three of the four Communication Scales: Trust, Interaction, and Satisfaction. The mean values obtained for the Communication - Accuracy Scale, as well as for all of the OS scales, are contained in Appendix $\mathrm{H}$.

Figure 4.24 presents the statistically significant differences obtained between the supervisory levels on the Communication - Trust Scale. The Supervisors had a statistically significantly higher mean value on this scale than the Non-Supervisors.

Statistically significant differences obtained between the SPR Supervisory Levels on the Communication - Interaction Scale are presented in Figure 4.25. The Supervisors had a statistically significantly higher mean value on this scale than the Non-Supervisors.

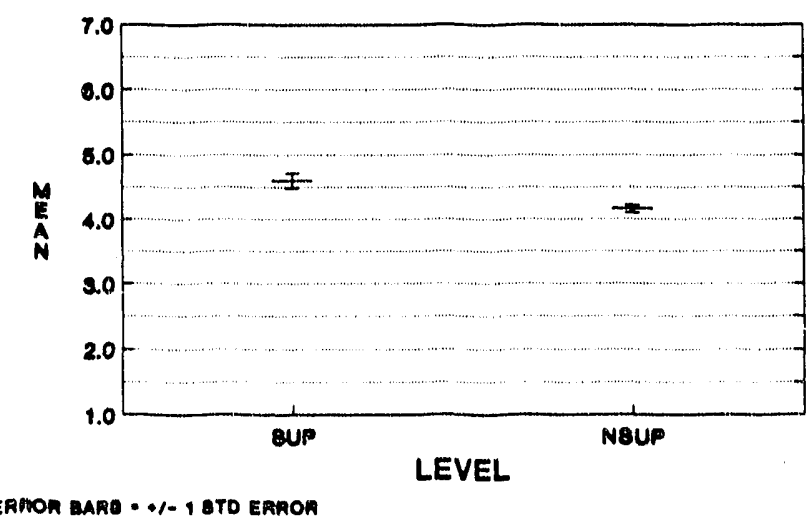

Figure 4.24. Significant differences between SPR supervisory levels on the communication-trust scale

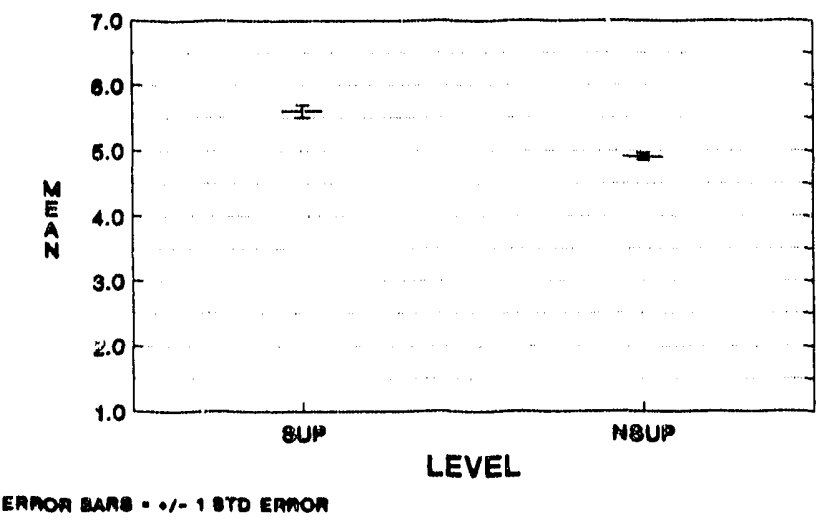

Figure 4.25. Significant differences between SPR supervisory levels on the communication-interaction scale

On the Communication - Satisfaction Scale, the Supervisors had a statistically significantly higher mean value than the Non-Supervisors. This difference is depicted in Figure 4.26. 


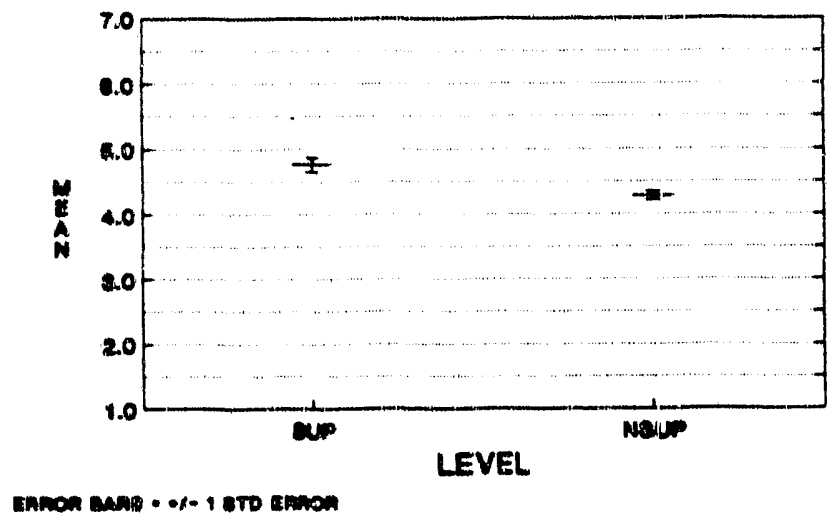

Figure 4.26. Significant differences between SPR supervisory levels on the communication-satisfaction scale

\subsubsection{Differences Between Supervisory Levels on the Additional Scales}

Statistically significant differences were obtained berween the SPR Supervisory Levels on three of the additional scales: Commitment, Cohesion, and Safety. Appendix $H$ contains the mean values obtained by each level on the other additional scales, as well as on all scales assessed by the OS.

Presented in Figure 4.27 are the statistically significant differences obtained between supervisory levels on the Commitment Scale. The Supervisors had a statistically significantly higher mean value on this scale than the Non-Supervisors.

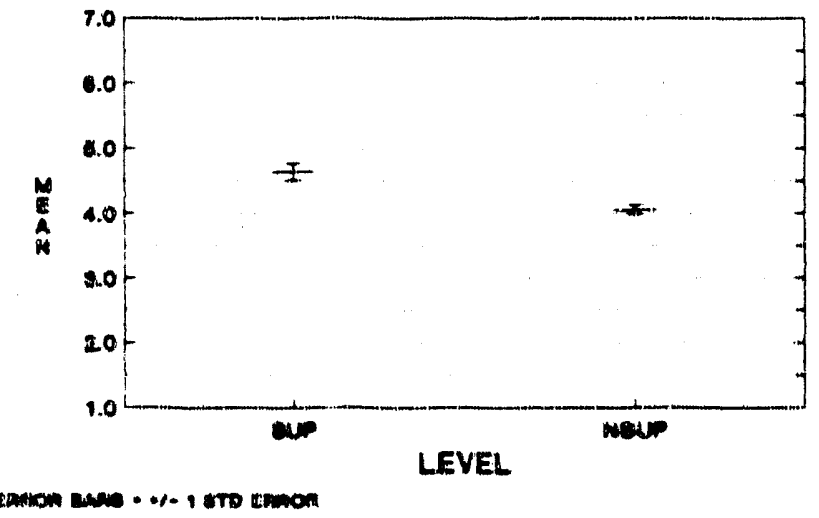

Figure 4.27. Significant differences berween SPR supervisory levels on the commitment scale

Figure 4.28 depicts the statistically significant differences obtained between the SPR Supervisory Levels on the Colvesion Scale. The Supervisors had a statistically significantly higher mean value on this scale than the Non-Supervisors. 


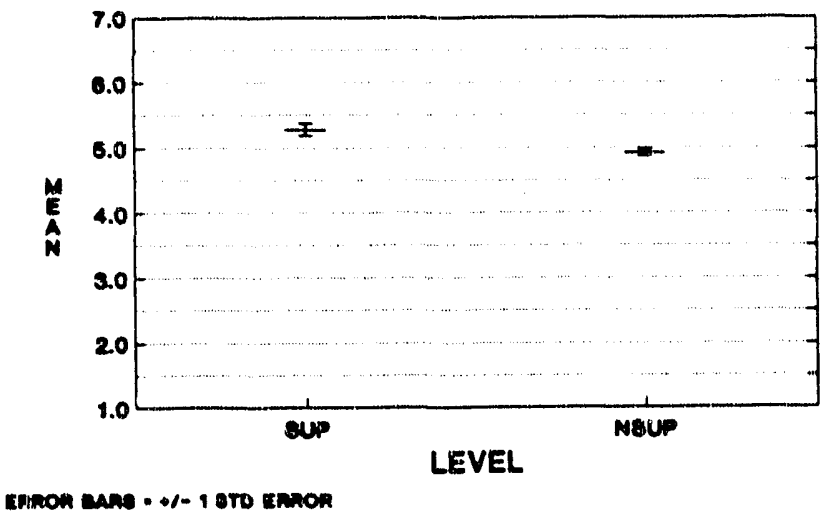

Figure 4.28. Significant differences between SPR supervisory levels on the cohesion scale

Statistically significant differences obtained between the SPR Supervisory Levels on the Safety Scale are presented in Figure 4.29. The Non-Supervisors had a statistically significantly lower mean value on this scale than the Supervisors.

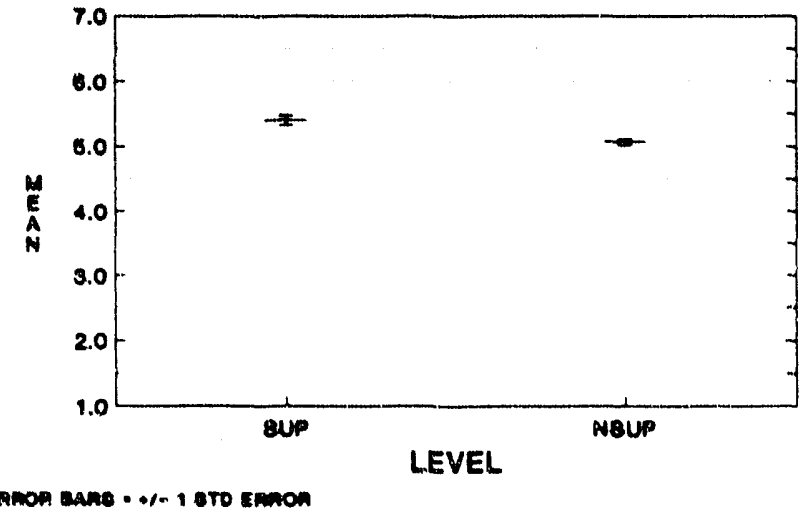

Figure 4.29. Significant differences between SPR supervisory levels on the safety scale

\subsubsection{Differences Between Supervisory Levels on the Environment, Safety, and Health Questions}

No statistically significant differences were obtained between SPR Supervisory Levels on the Environment, Safety, and Health Questions. Appendix $\mathrm{H}$ contains the mean values obtained for each level on these questions.

\subsubsection{Summary}

Statistically significant differences betiveen supervisory levels at SPR were obtained on numerous scales, and all differences appear to reflect differences in perceptions of expectations and not differences in function. Supervisors had higher mean values on the scales which make up the Constructive Cultural Style (Humanistic-Eacouraging (C1), Affiliative (C2), Achievement (C11), and Self-Actualizing (C12) Scales). Additionally, supervisors had statistically significantly higher mean values on three of the four 
Communication Scales (Trust, Interaction, and Satisfaction). They scored higher on commitment to the organization as well as cohesion within their working groups. Finally, supervisors perceive those behaviors which are believed to influence safety as being more helpful to them in doing their jobs well. While these results are generally consistent with what might be expected based on results obtained at other DOE facilities, the differences at SPR between supervisory levels are somewhat more numerous.

\subsection{Differences Between the Boeing Departments on the OS Scales}

\subsubsection{Differences Between the Boeing Departments on the $\mathrm{OCI}$}

No statistically significant differences were obtained between the Boeing Departments on any of the OCI Scales. Appendix I contains the mean values obtained for each department on each scale.

\subsubsection{Differences Between the Boeing Departments on the Communication Scales}

No statistically significant differences were obtained between the Boeing Departments on any of the Communication Scales. Appendix I contains the mean values obtained for each department on each scale.

\subsubsection{Differences Between the Boeing Departments on the Additional Scales}

Statistically significant differences between the Boeing Departments occurred on one of the additional scales: Hazard. The mean values obtained by each department for each of the scales are contained in Appendix I.

Figure 4.30 presents the statistically significant differences obtained between the Boeing Departments on the Hazard Scale. The Project Planning and Control Department had the lowest mean value on this scale and was statistically significantly different from the Operations and Maintenance, Technical Assurance, Engineering, and Project Management Departments. . The Operations and Maintenance Department had the highest mean value on this scale and was also statistically significantly different from the Business Management Department. Other statistically significant differences obtained between departments on the Hazard Scale are presented in Appendix I.

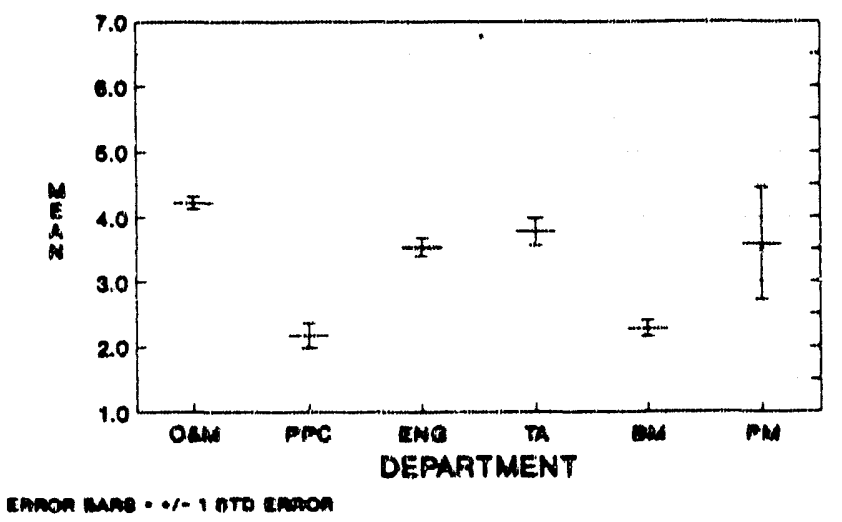

Figure 4.30. Significant differences between Boeing departments on the hazard scale 


\subsubsection{Differences Between Boeing Departments on the Environment, Safety, and Health Questions}

Statistically significant differences were obtained between the Boeing Departments on two of the Environment, Safety, and Health Questions: Offsite and Onsite Consequences. The mean values obtained by each department on the other Environment, SaF ry, and Health Questions are contained in Appendix 1 .

Figure 4.31 depicts the statistically significant differences obtained between the Boeing Departments on the Offsite Consequences Question. The Business Management Department had the lowest mean value on this question and, along with the Project Planning and Control Department, was statistically significantly different from the Operations and Maintenance, Engineering, and Technical Assurance Departments. Other statistically significant differences between Boeing Departments on this question are presented in Appendix I.

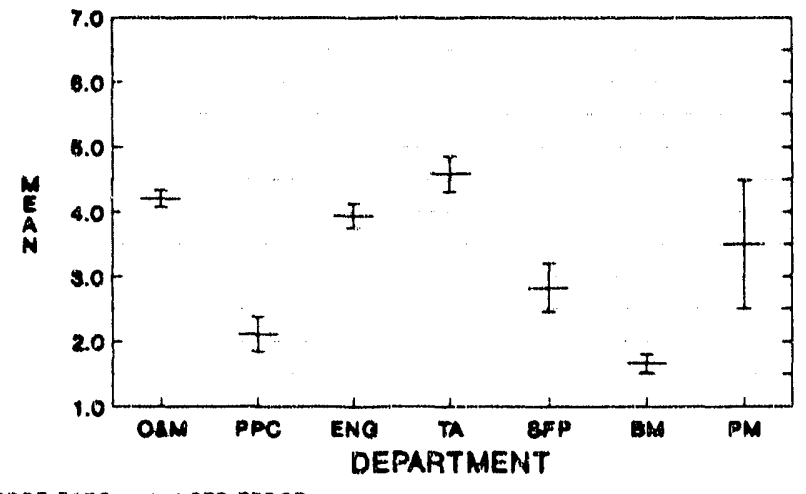

Figure 4.31. Significant differences between Boeing departments on the offsite consequences question

A similar pattern of differences is obtained when the Boeing Departments are compared on the Onsite Consequences Question (Figure 4.32). The Business Management Department had the lowest mean value and along with the Project Planning and Control Department was statistically significantly different from the Operations and Maintenance, Engineering, and Technical Assurance Departments. The Operations and Maintenance Department had the highest mean value on this question. Other statistically significant differences between the Boeing Departments on this question are presented in Appendix I.

\subsubsection{Summary}

The statistically significant differences obtained between the Boeing Departments were all on scales/questions which relate to the hazardous/environmentally consequential nature of their work. Thus, the differences obtained appear to reflect differences in functional roles within the organization. 


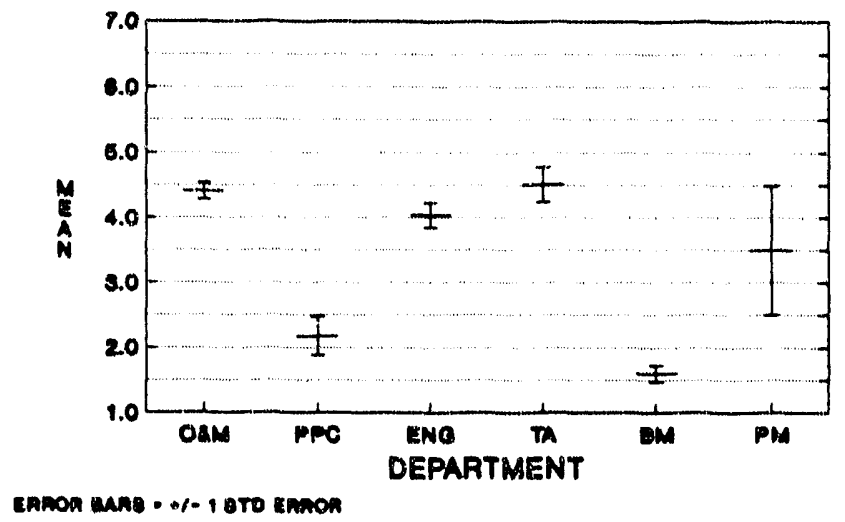

Figure 4.32. Significant differences between Boeing departments on the onsite consequences question

\subsection{Differences Between DOE Departments on the OS}

\subsubsection{Differences Between DOE Departments on the OCI}

No statistically significant differences were obtained between the DOE Departments on the OCl. Appendix $\mathrm{J}$ contains the mean values obtained on each of these scales by each department.

\subsubsection{Differences Between DOE Departments on the Communication Scales}

No statistically significant differences were obtained between the DOE Departments on any of the Communication Scales. Appendix J presents the mean values obtained by each department on each Communication Scale.

\subsubsection{Differences Between DOE Departments on the Additional Scales}

No statistically significant differences were obtained when the DOE Departments were compared on any of the additional scales. Presented in Appendix $J$ are the mean values obtained by each DOE Department on each scale.

\subsubsection{Differences Between DOE Departments on the Environment, Safety, and Health Questions}

Statistically significant differences between DOE Departments were obtained on one of the Environment, Safety, and Health Questions: Onsite Consequences. The mean values obtained by each DOE Department on each of the other questions are presented in Appendix J.

Figure 4.33 depicts the statistically significant differences obtained between the DOE Departments on the Onsite Consequences Question. The Operations Department had the highest mean value, and along with the Environment, Safety, and Health Department, were statistically significantly different from the Project Manager's Office. The Project Manager's Office had the lowest mean value on this question. The Operations Department also had a statistically significantly higher mean value on this question than the Management and Administration Department. No other statistically significant differences were obtained between the DOE Departments on this question. 


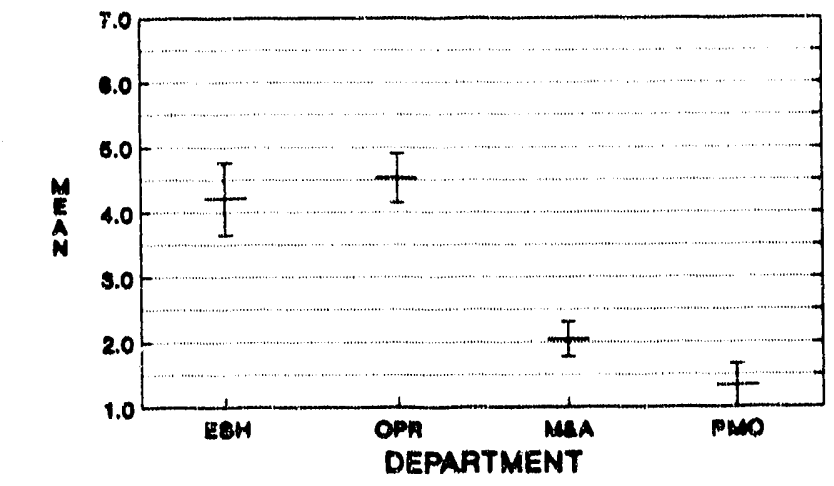

Ennon aAne - +/- 1 gto Ennon

Figure 4.33. Significant differences between DOE departments on the onsite consequences question

\subsubsection{Summary}

Statisticaliy significant differences between the DOE Departments were obtained only on the Onsite Environmental Consequences Question. Thus, it appears that the DOE organization is comprised of various departments, all of which hold similar perceptions in terms of the expectations they believe are placed on them by their management.

\subsection{Differences Between the Boeing Employee Categories on the OS}

\subsubsection{Differences Between the Boeing Employee Categories on the OCI}

No statistically significant differences were obtained between the Boeing Employee Categories on any of the OCI Scales. Appendix $\mathrm{K}$ presents the mean values obtained by each category on each of the OCI Scales.

\subsubsection{Differences Between the Boeing Employee Categories on the Communication Scales}

No statistically significant differences were obtained between the Boeing Employee Categories on any of the Communication Scales. Appendix $\mathrm{K}$ presents the mean values obtained by each Boeing Employee Category on each of the Communication Scales.

\subsubsection{Differences Between the Boeing Employee Categories on the Additional Scales}

Statistically significant differences between the Boeing Employee Categories occurred on one of the additional scales: Hazard. The mean values obtained by each Boeing Employee Category on each of the other additional scales are contained in Appendix $\mathrm{K}$.

Presented in Figure 4.34 are the statistically significant differences obtained between the Boeing Employee Categories on the Hazard Scale. The Hourly employees had the highest mean value on this scale and were statistically significantly different from both the Exempt and Non-Exempt Employee Categories. The Non-Exempt category had the lowest mean value on this scale and was also statistically significantly different from the Other category. 


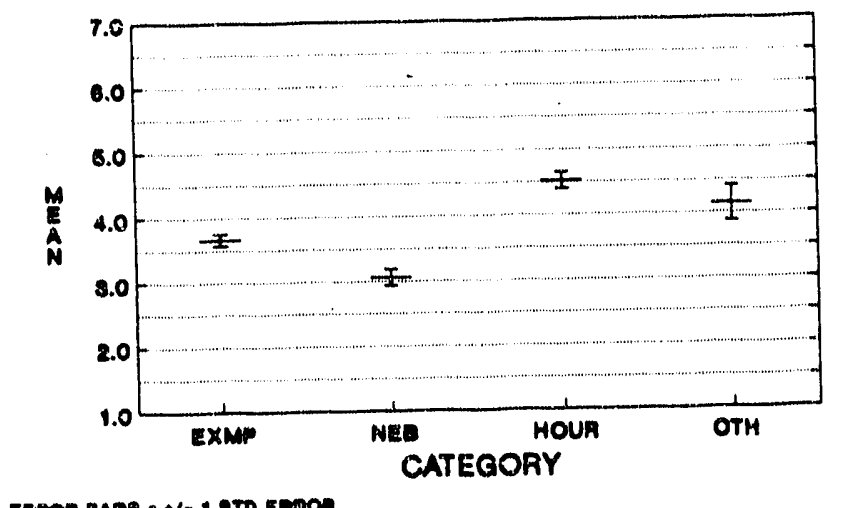

Figure 4.34. Significant differences between Boeing employee categories on the hazard scale

4.9.4 Differences Between Boeing Einployee Categories on the Environment, Safety, and Health Question

Statistically significant differences between the Boeing Employee Categories were obtained on two of the Environment, Safety, and Health Questions: Offsite and Onsite Consequences. The mean values obtained by each category on the other questions are contained in Appendix $\mathrm{K}$.

Figure 4.35 depicts the statistically significant differences obtained between the Boeing Employee Categories on the Offsite Environmental Consequences Question. The Hourly category had the highest mean value on this question and was statistically significantly different from the Non-Exempt Boeing Employee Category. No other differences were obtained on this question.

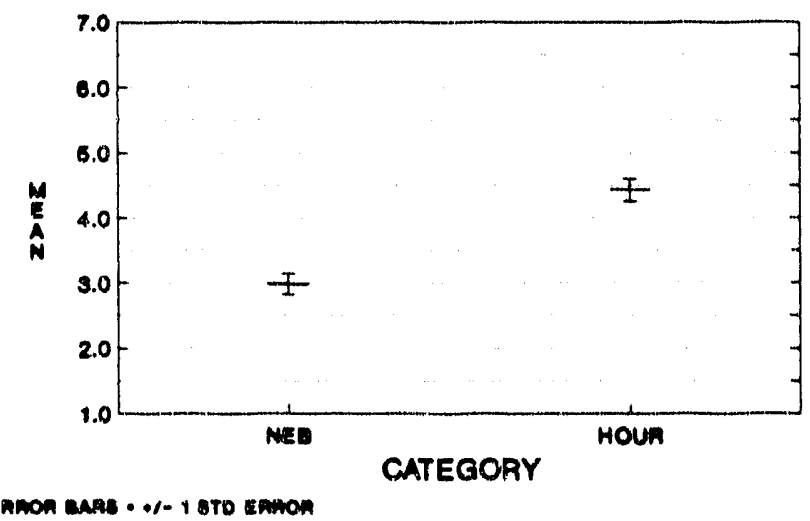

Figure 4.35. Significant differences between Boeing employee categories on the offsite consequences question

Statistically significant differences obtained between the Boeing Employee Categories on the Onsite Consequences Question are depicted in Figure 4.36. The Hourly Boeing Employee Category had the highest mean value on this scale and, along with the Other Boelug Employee Category, was 
statistically significantly different from the Non-Exempt Boeing Employee Category. No other statistically significant differences were obtained between the Boeing Employee Categories on this question.

\subsubsection{Summary}

Few statistically significant differences were obtained between the Boeing Employee Categories on the OS. Those differences which were obtained were all related to the hazardous/environmentally consequential nature of one's work. In all instances, the Hourly Boeing Employee Category had the highest obtained mean values. The Non-Exempt Boeing Employee Category had the lowest obtained mean values. The differences thus appear to be consistent with work function.

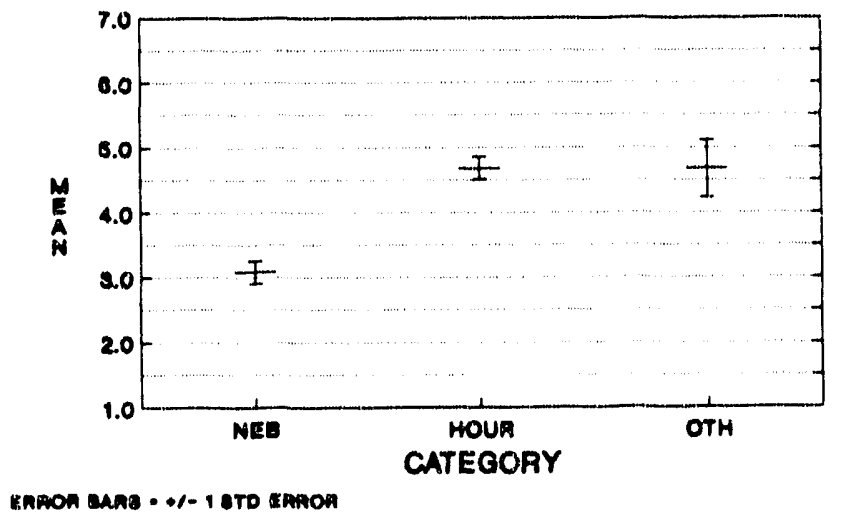

Figure 4.36. Significant differences between Boeing employee categories on the onsite consequences question

\subsection{Differences Between DOE Employee Categories on the OS}

\subsubsection{Differences Between DOE Employee Categories on the OCI}

No statistically significant differences were obtained between the DOE Employee Categories on the OCI Scales. Appendix L presents the mean values obtained by each category on each scale.

\subsubsection{Differences Between DOE Employee Categories on the Communication Scales}

No statistically significant differences were obtained between the DOE Employee Categories on any of the Communication Scales. Appendix $L$ contains the mean values obtained by each DOE Employee Category on each of these scales.

\subsubsection{Differences Between DOE Employee Categories on the Additional Scales}

No statistically significant differences were obtained between the DOE Employee Categories on any of the additional scales. Presented in Appendix $L$ are the mean values obtained by each DOE Employee Category on each of these scales. 
4.10.4 Differences Between DOE Employee Categories on the Environment, Safety, and Health Questions

No statistically significant differences were obtained when the DOE Employee Categories were compared on the Environment, Safety and Health Questions. Appendix $L$ presents the mean values obtained by each category on each of the questions.

\subsubsection{Summary}

No statistically significant differences were obtained between the DOE Employee Categories on any of the OS Scales. The lack of differences obtained here, in combination with the lack of differences found between the DOE Departments is further evidence for homogeneity within the DOE organization.

\subsection{Differences Between Boeing Supervisory Levels on the OS}

\subsubsection{Differences Between Boeing Supervisory Levels on the OCI}

Statistically significant differences obtained between the Boeing Supervisory Levels on the Humanistic-Encouraging (C1) Scale are presented in Figure 4.37. The Supervisors had a statistically significantly higher mean value on this scale than the Non-Supervisors.

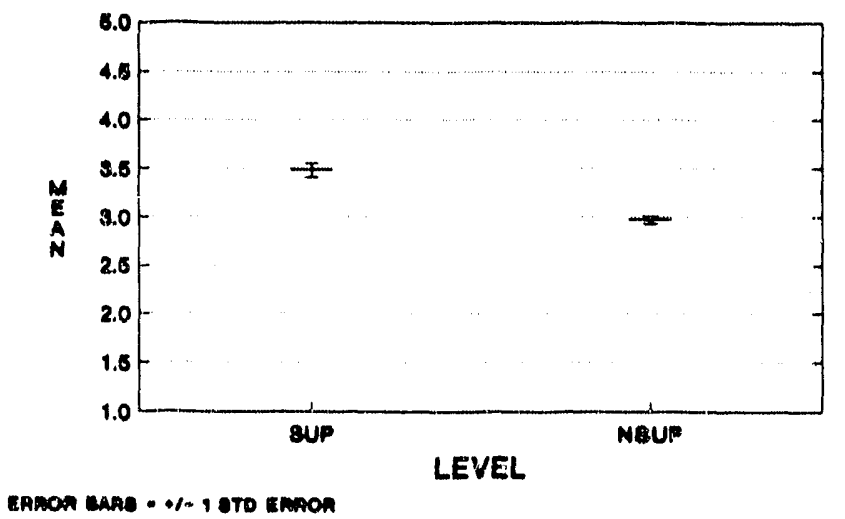

Figure 4.37. Significant differences between Boeing supervisory levels on the humanistic-encouraging scale

Figure 4.38 depicts the statistically significant differences which were obtained between the Boeing Supervisory Levels on the Affiliative (C2) Scale. The Supervisors had a statistically significantly higher mean value on this scale than the Non-Supervisors.

No statistically significant differences were obtained between the Boeing Supervisory Levels on the Approval (C3) Scale. The mean values obtained by each level on this scale, as well as on all of the OS Scales, are presented in Appendix $M$.

Statistically significant differences obtained between the Boeing Supervisory Levels on the Conventional (C4) Scale are depicted in Figure 4.39. The Non-Supervisors had a statistically significantly higher mean value on this scale than the Supervisors. 


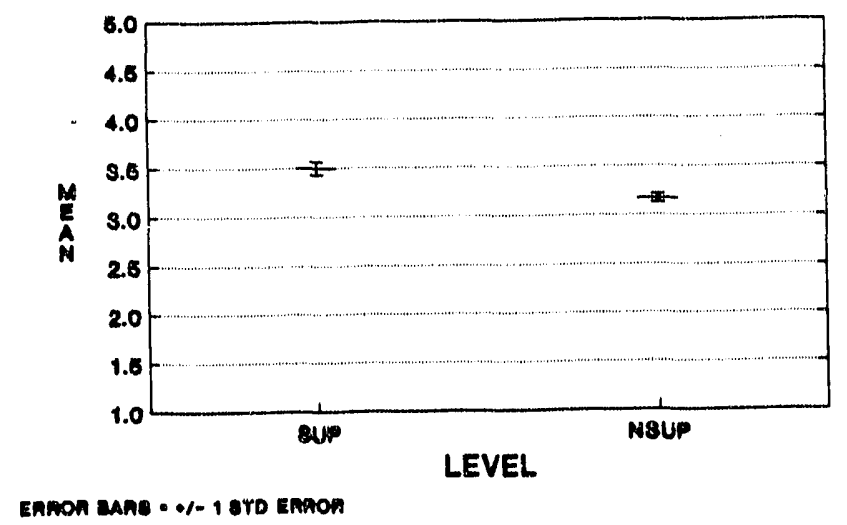

Figure 4.38. Significant differences between Boeing supervisory levels on the affiliative scale

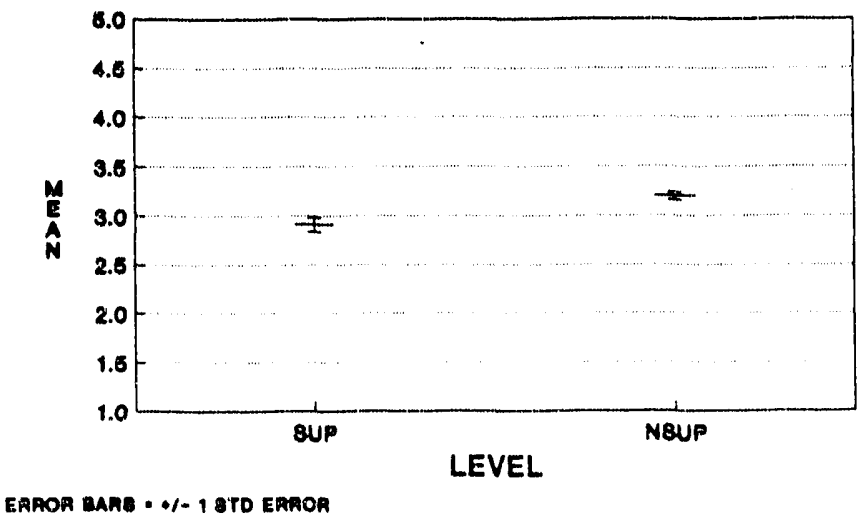

Figure 4.39. Significant differences between Boeing supervisory levels on the conventional scale

Statistically significant differences were obtained between the Boeing Supervisory Levels on the Dependent (C5) Scale (Figure 4.40). The Non-Supervisors had a statistically significantly higher mean value on this scale than the Boeing Supervisors.

No statistically significant differences were obtained between the Boeing Supervisory Levels on the Avoidance (C6), Oppositional (C7), Power (C8), Competition (C9), or Perfectionistic (C10) Scales. Appendix $M$ contains the mean values obtained by each Boeing Supervisory Level on each of these scales.

Figure 4.41 depicts the statistically significant differences obtained between the Boeing Supervisory Levels on the Achievement (C11) Scale. The Supervisors had a statistically significantly higher mean value on this scale than the Non-Supervisors.

Presented in Figure 4.42 are the statistically significant differences obtained between the Boeing Supervisory Levels on the Self-Actualizing (C12) Scale. The Supervisors at Boeing had a statistically significantly higher mean value on this scale than the Non-Supervisors. 


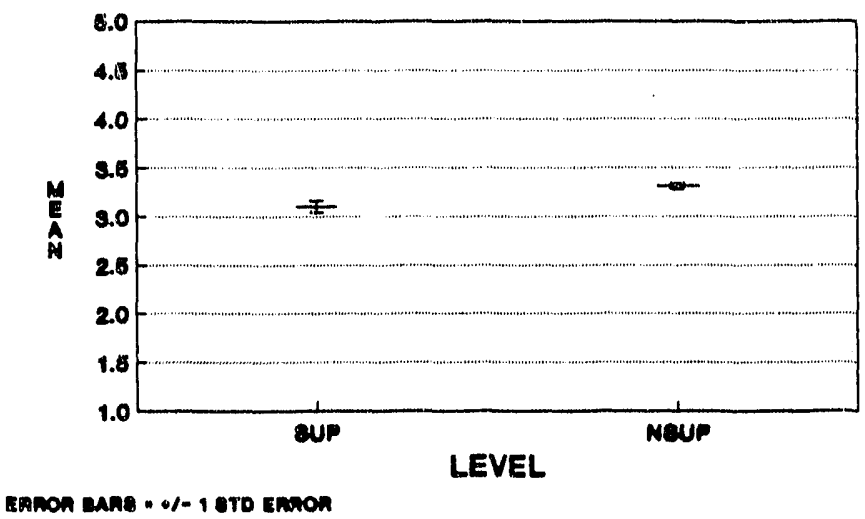

Figure 4.40. Significant differences between Boeing supervisory levels on the dependent scale

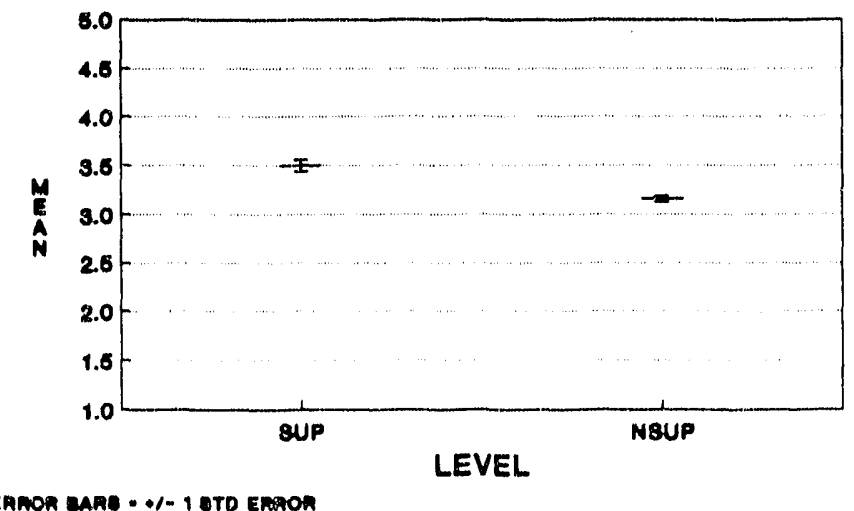

Figure 4.41. Significant differences between Boeing supervisory levels on the achievement scale

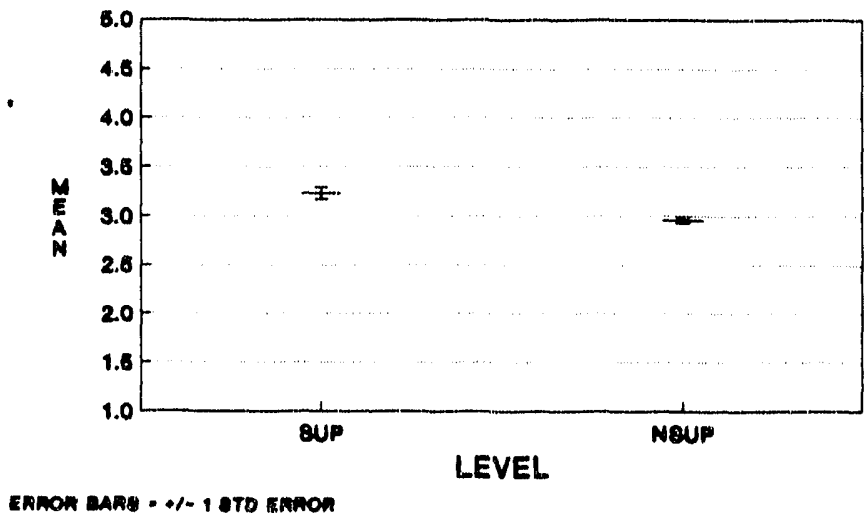

Figure 4.42. Significant differences between Boeing supervisory levels on the self-actualizing scale 
4.11.2 Differences Between the Boeing Supervisory Levels on the Communication Scales

Statistically significant differences were obtained between the Boeing Supervisory Levels on three of the four Communication Scales: Trust, Interaction, and Satisfaction. The mean values obtained for each supervisory level on the Communication - Accuracy Scale are contained in Appendix M.

Figure 4.43 depicts the statistically significant differences obtained between the Boeing Supervisory Levels on the Communication - Trust Scale. The Supervisors had a statistically significantly higher mean value on this question than the Non-Supervisors.

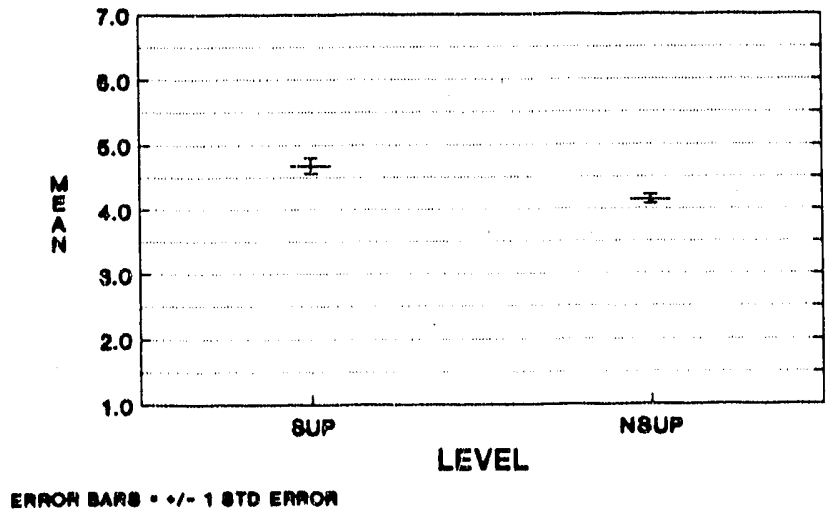

Figure 4.43. Significant differences between Boeing supervisory levels on the communication-trust scale

Presented in Figure 4.44 are the statistically significant differences obtained between the Boeing Supervisory Levels on the Communication - Interaction Scale. The Boeing Supervisors had a statistically significantly higher mean value on this scale than the Boeing Non-Supervisors.

Statistically significant differences between the Boeing Supervisory Levels on the Communication - Satisfaction Scale are depicted in Figure 4.45. The Supervisors had a statistically significantly higher mean value on this scale than the Non-Supervisors.

\subsubsection{Differences Between the Boeing Supervisory Levels on the Additional Scales}

Statistically significant differences were obtained between the Boeing Supervisory Levels on four of the additional scales: Commitment, Cohesion, Safety, and Job Satisfaction. The mean values obtained by each level on the other additional scales are contained in Appendix M.

Figure 4.46 depicts the statistically significant differences obtained between the Boeing Supervisory Levels on the Commitment Scale. The Supervisors had a statistically significantly higher mean value than the Non-Supervisors on this scale.

On the Cohesion, the Safety, and the Job Satisfaction Scales, the Boeing Supervisors had statistically significantly higher mean values than the Boeing Non-Supervisors. These differences are depicted in Figures 4.47, Figure 4.48, and Figure 4.49, respectively. 


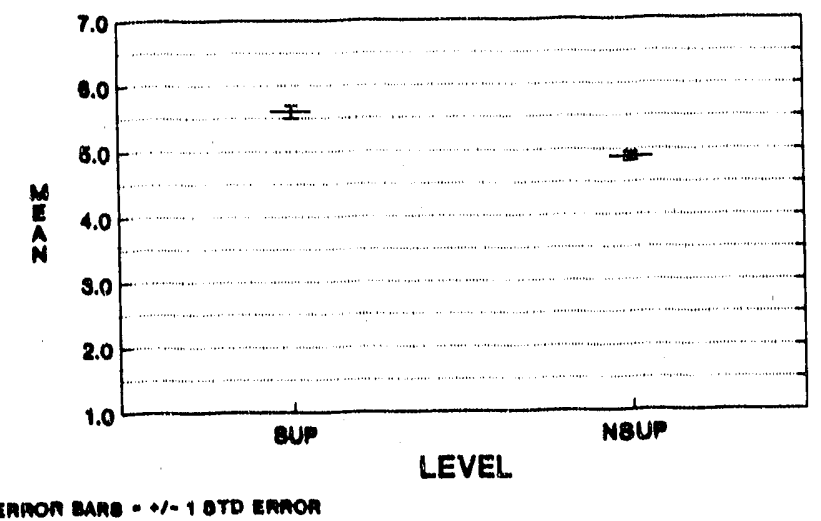

Figure 4.44. Significant differences between Boeing supervisory levels on the communication-interaction scale

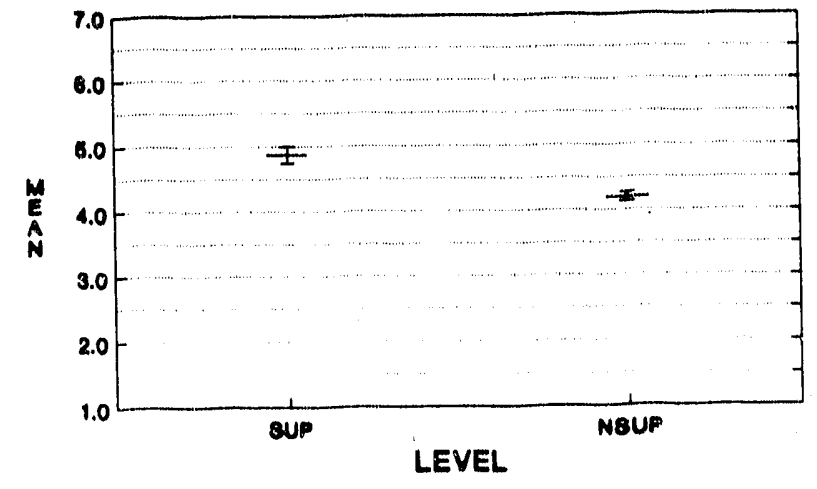

Ennon anne - */- 1 vro smon

Figure 4.45. Significant differences between Boeing superviscry levels on the communication-satisfaction scale

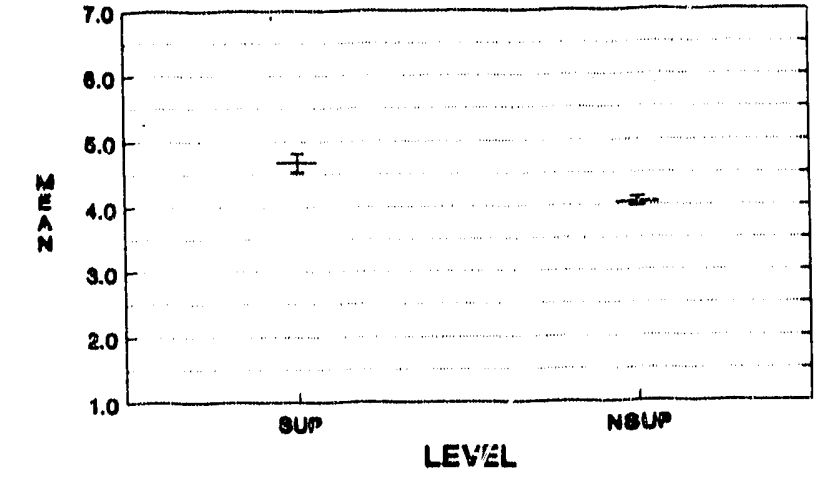

capon eane - *1- 1 sto temon

Figure 4.46. Significant differences between Boeirg supervisory levels on the commitment scale 


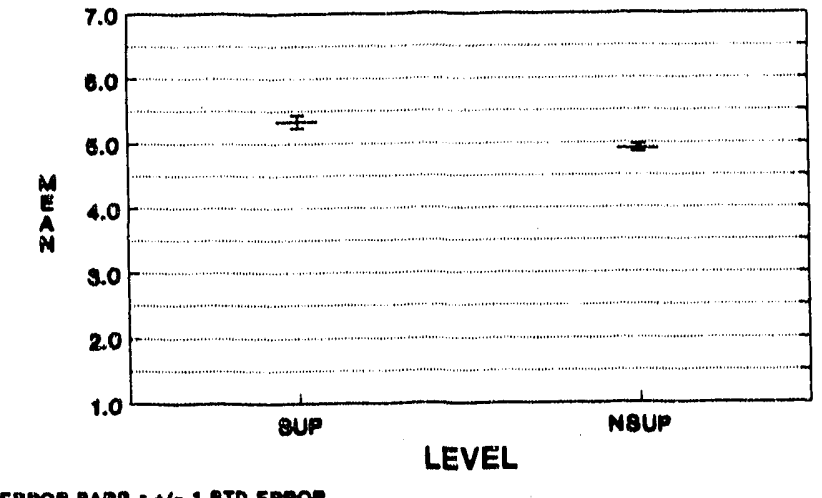

Figure 4.47. Significant differences between Boeing supervisory levels on the cohesion scale

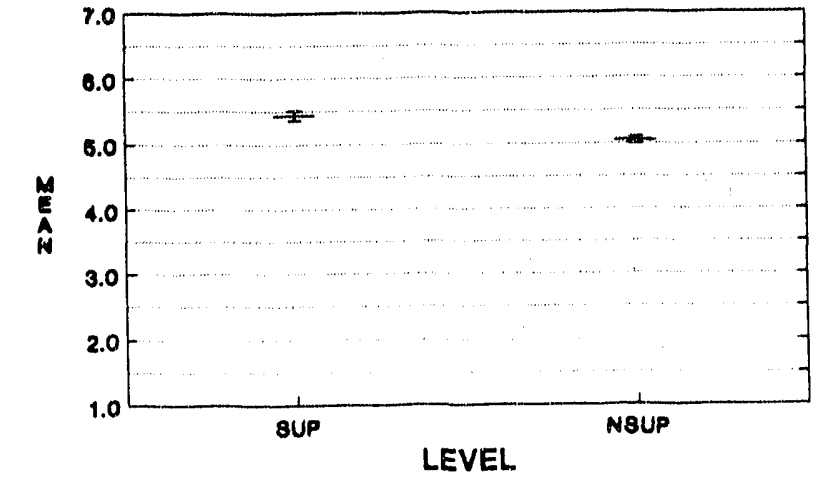

EAAOA BARB - +/- 1 9TO ERAOR

Figure 4.48. Significant differences between Boeing supervisory levels on the safety scale

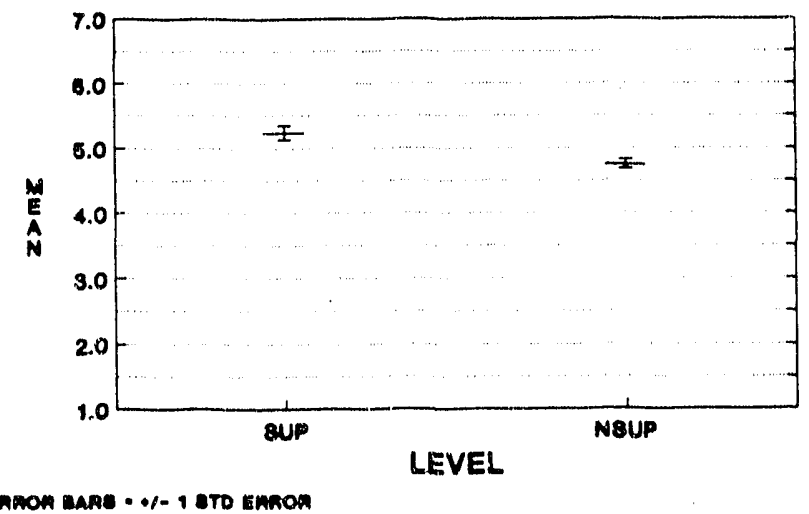

Figure 4.49. Significant differences between Boeing supervisory levels on the job satisfaction scale 
4.11.4 Differences Between the Boeing Supervisory Levels on the Environiment, Safety, and Health Questions

No statistically significant differences were obtained between the Boeing Supervisory Levels on asiy of the Environment, Safety, and Health Questions. The mean values obtained by each level on each of these questions are presented in Appendix $M$.

\subsubsection{Surnmary}

Numerous statistically significant differences were obtained between the Boeing Supervisory Levels on the OS scales. The differences are more numerous within the Boeing Organization than when the Supervisors and Non-Supervisors of the entire SPR were compared to each other. The differences, while consistent with the pattern of results obtained at other DOE facilities, are more numerous and appear to reflect differences in the perceptions of expectations, rather than differences in functional roles.

\subsection{Differences Between the DOE Supervisory Levels on the OS}

\subsubsection{Differences Between the DOE Supervisory Levels on the $\mathrm{OCI}$}

No statistically significant differences were obtained between the DOE Supervisory Levels on the $\mathrm{OCI}$ Scales. Appendix $\mathrm{N}$ contains the mean values obtained by each level on each of the scales.

\subsubsection{Differences Between the DOE Supervisory Levels on the Communication Scales}

No statistically significant differences were obtained between the DOE Supervisory Levels on any of the Communication Scales. The mean values obtained by each level on each of the Communication Scales are contained in Appendix $N$.

\subsubsection{Differences Between the DOE Supervisory Levels on the Additional Scales}

No statistically significant differences were obtained between the DOE Supervisory Levels on any of the additional scales. Appendix $N$ presents the mean values obtained by each DOE supervisory level on each of the additional scales.

4.12.4 Differences Berween the DOE Supervisory Levels on the Environment, Safety, and Health Questions

No statistically significant differences were obtained between the DOE Supervisory Levels on any of the Environment, Safety, and Health Questions. The mean values obtained by each level on each question are contained in Appendix $\mathrm{N}$.

\subsubsection{Summary}

No statistically significant differences were found between DOE Supervisors and Non-Supervisors at SPR on any of the OS Scales. Once again, this lends further support to the seemingly homogeneous culture which exists within the DOE Organization ai SPR. 


\section{CONCLUSIONS}

The Organizational Survey (OS) which took place at the Strategic Petroleum Reserve (SPR) on November 4 through 8, 1991 was the thirteenth to occur at a U.S. Department of Energy (DOE) facility. This administration, however, was different from the other twelve administrations in that it occurred at the request of SPR management prior to their Tiger Team Assessment. Their intention was to utilize the findings of the survey in their self-assessment process. The survey will be readministered at a later date which will allow an assessment of change management to be made.

All 1224 Boeing and DOE employees received notice of the OS administration. The administration occurred at all eight of the SPR Sites. A total of 879 employees actually completed the survey, yielding a response rate of 71.8 percent. The response rate obtained for the Boeing Organization was 64.0 percent, while the response rate obtained for the DOE Organization was 94.8 percent. Response rates by department varied from a high response rate of 116.7 percent for the Project Planning and Control Department to a low of 50.9 percent for the Operations and Maintenance Department.

The profile obtained on the scales from the Organizational Culture Inventory (OCI) (Human Synergistics, 1987), indicates that the SPR organization may be inhibited from achieving their work goals in a constructive and creative manner due to behavioral expectations which include the need for approval (C3), conventionalism (C4), and dependence (C5). These scales comprise the Passive-Defensive Cultural Style. There are some expectations for creative, constructive, and team-oriented behavicr, based on mean values on the Humanistic-Encouraging (C1), Affiliative (C2), Achievement (C11), and Self-Actualizing (C:2) Scales. However, these expectations are overshadowed by the more passive-defensive behaviors discussed earlier. Additionally, relatively low mean values were obtained on the Communication - Trust and Communication -Accuracy Scales, a further indication of a potentially defensive culture. The perception also exists that working units are not well coordinated. Employees perceive a low amount of hazard and environmental consequences to exist at the SPR, although they do believe those attributes important to safety are helpful in doing one's job well.

No differences were obtained between the Boeing and DOE Organizations at SPK on any of the OS Scales. This suggests that the overall SPR conclusions are also appropriate to both the Boeing and DOE Organizations. This finding is surprising given the different functional roles these two organizations hold at SPR.

Differences were also obtained on the OS when the eight locations of SPR were compared to one another. No statistically significant differences were obtained between locations on either the $\mathrm{OCl}$ or Communication Scales, indicating that all locations were similar on these issues. However, results obtained on the additional scales and the Environment, Safety, and Health Questions indicate that the New Orleans Location is a consistent outlier, scoring lower on the Cohesion, Hazard, Safety, and Coordination Scales. They also scored lower on the Offsite Consequences, Onsite Consequences, Management Emphasis, and Employee Awareness Questions. Note, however, that the majority of these scales involve issues of hazard or environmental consequences. These differences may therefore arise from the different function the New Orleans Location fills within the SPR Organization. While differences between the remaining seven locations do not reveal other such extreme outliers, the Bryan Mound Location scored lower than other locations on both the Cohesion and Coordination Scales. They are also the only location which was not different from the New Orleans Location on the Management Emphasis Question. The Weeks Island Location is lower from some of the other locations on the Coordination Scale, and the West Hackberry Location is lower than other locations on the Cohesion 
Scale. From the seven locations, the one which appears to be the most different from the New Orleans Location is the Sulphur Mines Location.

Differences between employee categories at SPR were not numerous. All but one of the differences appear to reflect differences in job functions, particularly as related to the hazardousness associated with one's job.

Differences obtained between supervisory levels were quite numerous, perhaps even more numerous than might be expected, although all were consistent with results obtained at other DOE facilities. The differences appear to reflect differences in perceptions of expectation (i.e., OCI Scales, Communication Scales, Commitment, Cohesion, and Safety Scales) rather than function (i.e. Hazard and Coordination Scales, Environment, Safety, and Health Questions).

Differences within the Boeing Organization, particularly between departments and employee categories, generally appear related to the functional roles various groups play within the organization. All differences related to departments and employee categories within Boeing were on the Hazard Scale and the Environment, Safety, and Health Questions. Differences between Boeing Supervisors and NonSupervisors were even more numerous than between the overall SPR Supervisors and Non-Supervisors and did not appear related to the functional role of Supervisors and Non-Supervisors within Boeing.

Only one difference was obtained within the DOE Organization and this occurred on the Onsite Environmental Consequences Question when the DOE Departments were compared to one another. No differences were obtained between DOE Employee Categories or Supervisory Levels. This suggests that the DOE organization is very homogeneous with respect to the issues addressed in this survey.

In summary, SPR, as represented by the sample of employees who completed the Organizational Survey, is an organization which, tends to place emphasis on behaviors which are considered as "passive defensive". No differences were obtained between the DOE and Boeing Organizations, and therefore, the conclusion of a passive-defensive type culture appears to apply well to both organizations. Differences between the SPR Locations were not numerous and appeared only on the additional scales and the Environment, Safety, and Health Questions. The differences which were obtained indicated that the New Orleans Location is an obvious outlier relative to the other locations. Differences between the SPR Employee Categories were few and appear related to job function. Differences between Supervisors and Non-Supervisors were more numerous, although consistent, with what might be expected. Differences within the Boeing Organization were few and seem related to function for all analyses except those for supervisory level. Only one difference was found within the DOE Organization. These findings suggest that the DOE Organization is fairly homogeneous. 


\section{REFERENCES}

Cooke, R.A., and Burack, E.H., "Measuring Norms and Expectations with the OCI," in Organizational Culture Inventory, Level V Manual, Chicago: Human Synergistics, 1987, pp. 13-26.

Hays, W.L., Statistics, Fourth Edition, New York: Holt, Rinehart, and Winston, Inc., 1988.

Human Synergistics, Organizational Culture Inventory Level V, Plymouth, MI, 1987.

Georgopolous, B.S., and Mann, F.C., The Community General Hospital, New York: Macmillan, 1962.

Kunin, J., "The construction of a new type of attitude measure," Personnel Psychology, 8 , 1955, pp. 65-78.

Mowday, R., and Steers, R.M., The measurement of organizational commitment, Journal of Vocational Behavior, 14, 1979, pp. 224-247.

Price, J.L., and Muller, Handbook of Organizational Measurement, Lexington, MA: DC Heath and Company, 1972.

Roberts, K.M., and O'Reilly, C.A., "Measuring organizational communications," Journal of Applied Psychology, 59 (3), 1974, pp. 321-326 (copyright pending).

Seashore, S.E., Group Cohesiveness in the Industrial Work Group, Ann Arbor, MI: Survey Research Center, University of Michigan, MI, 1954. 

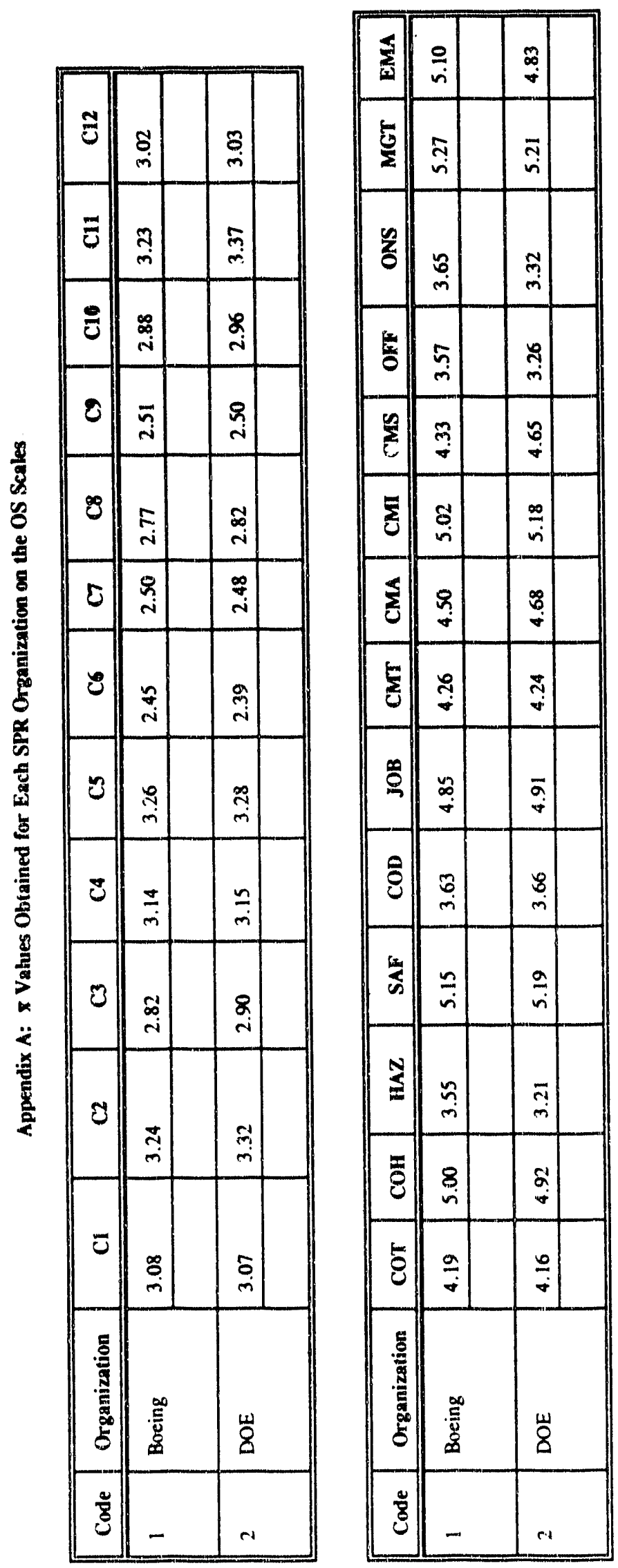

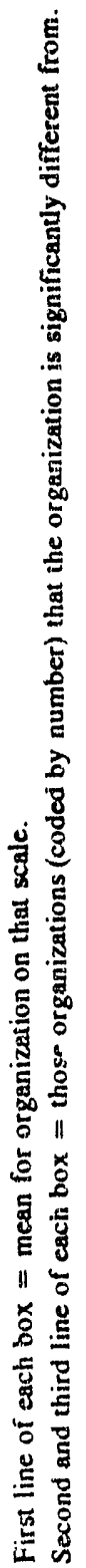




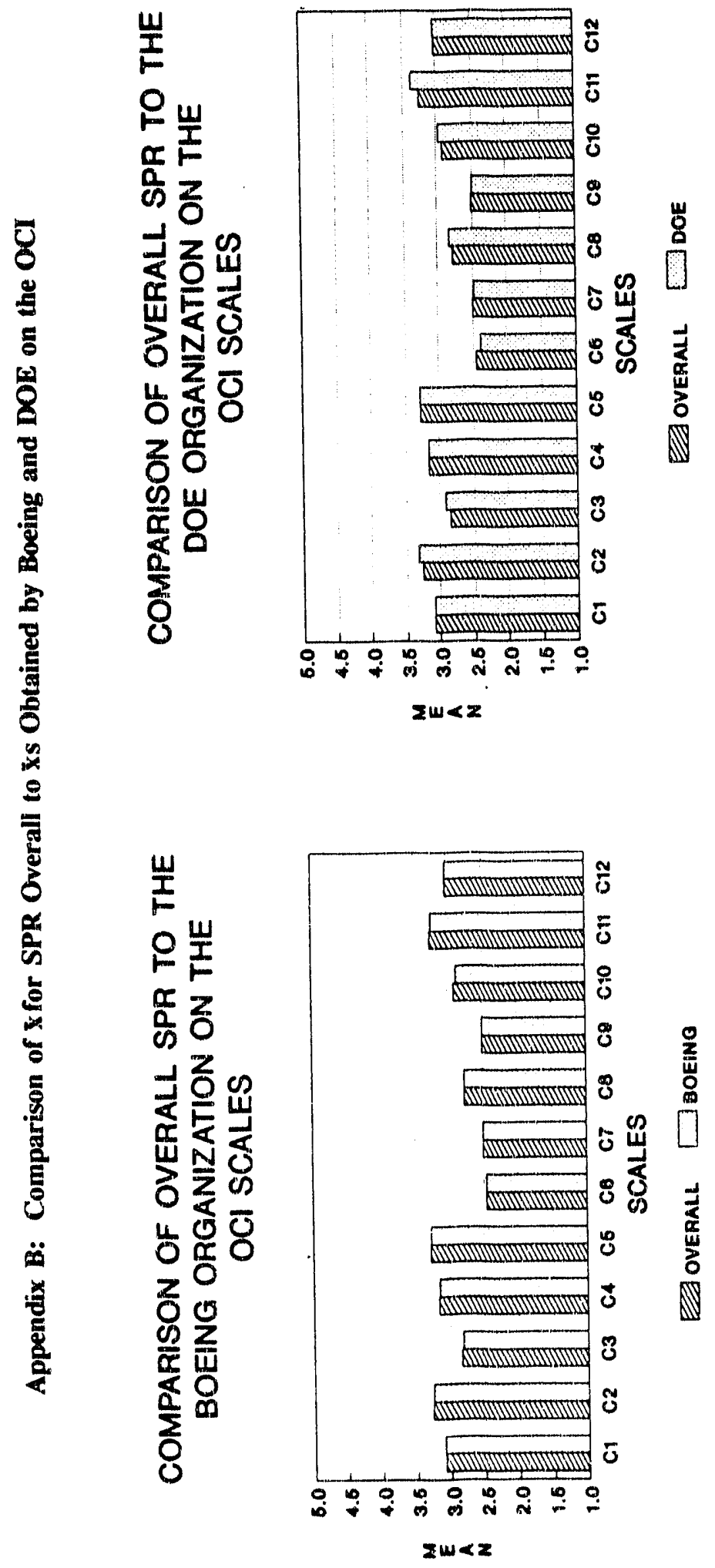




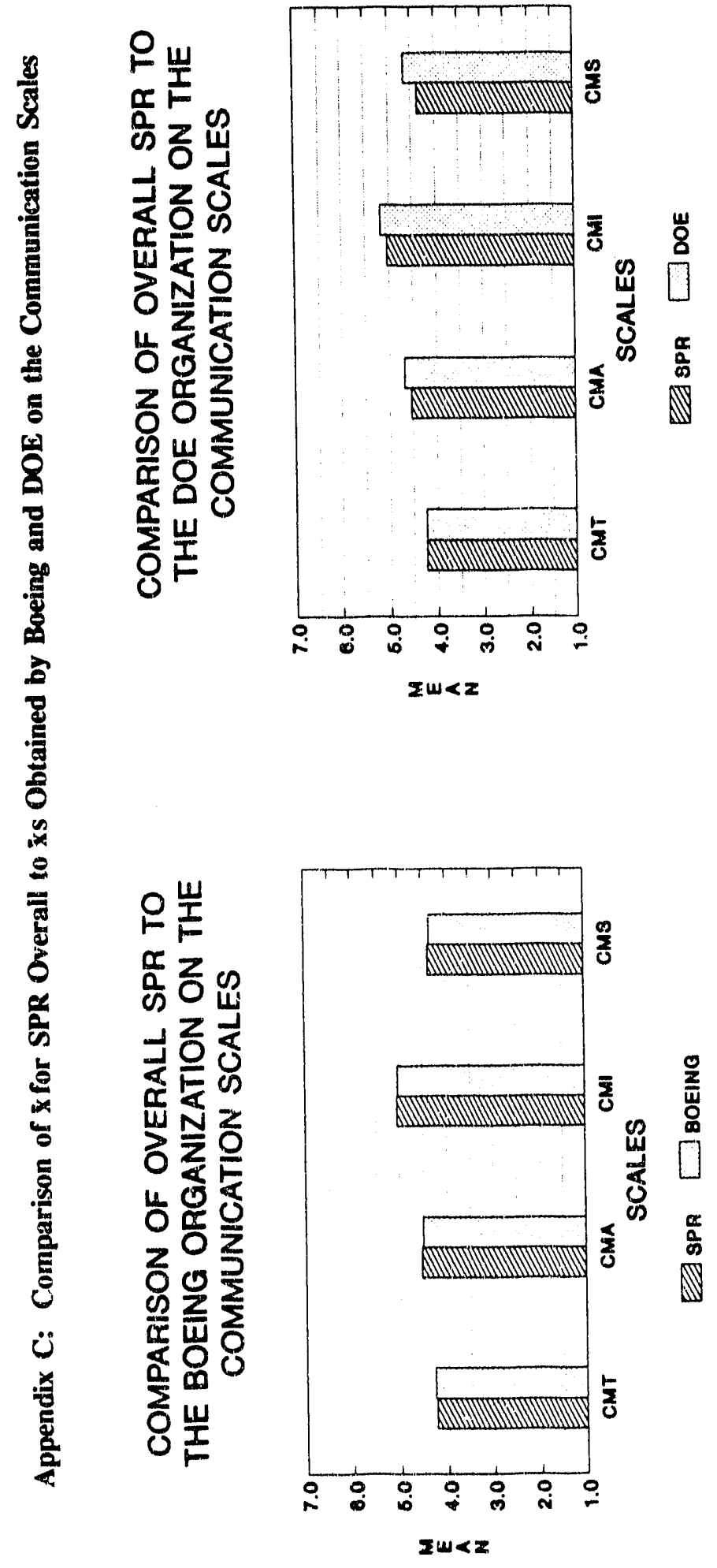




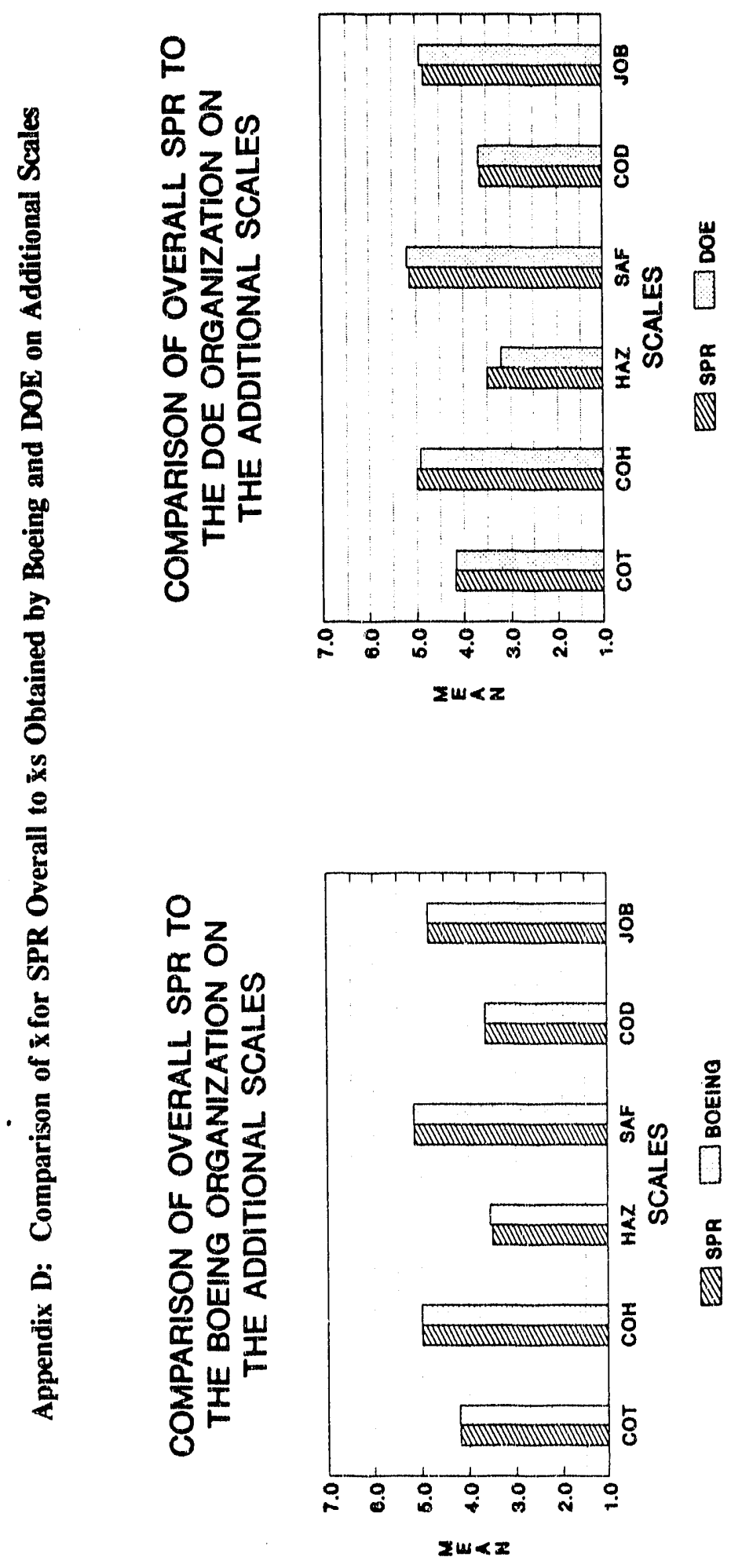




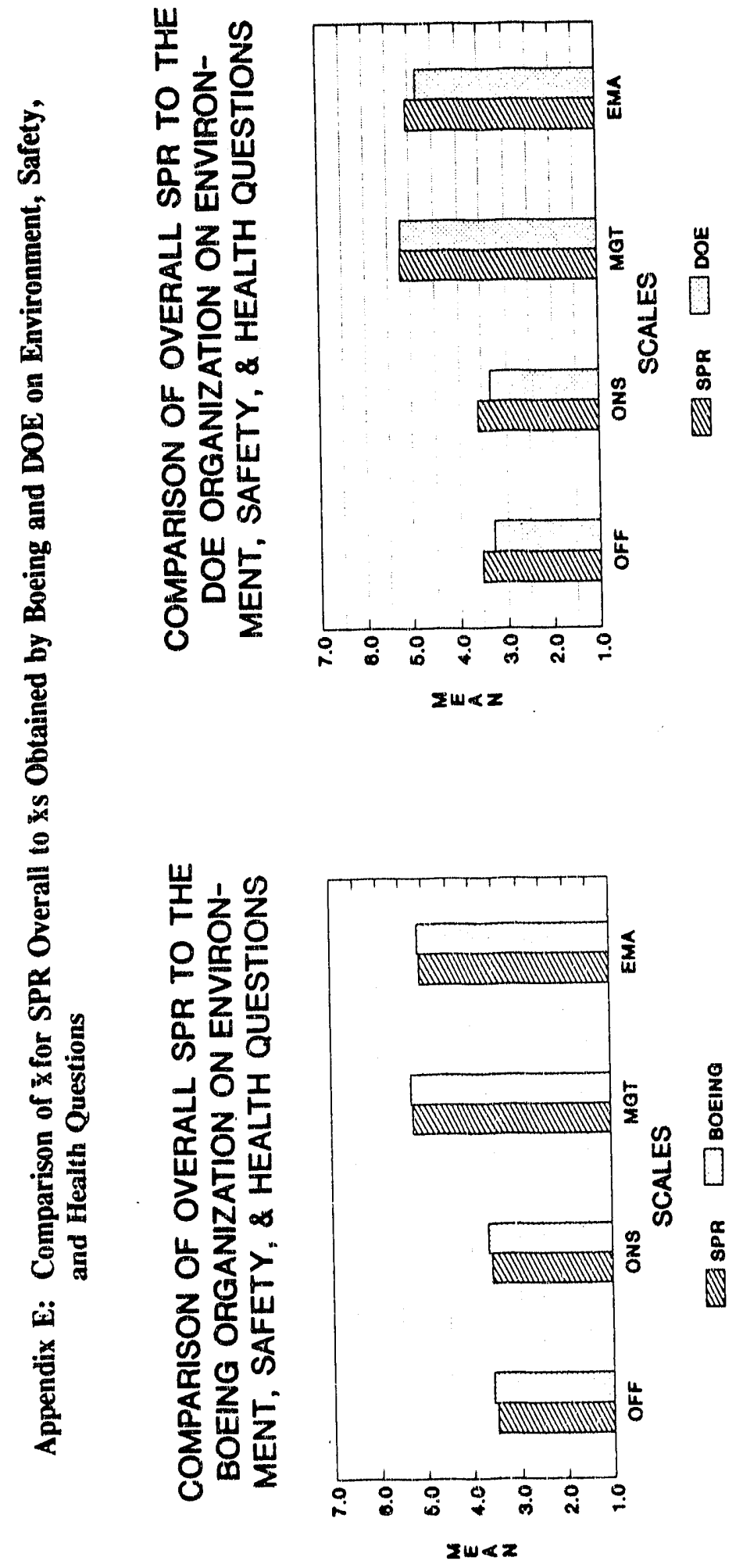




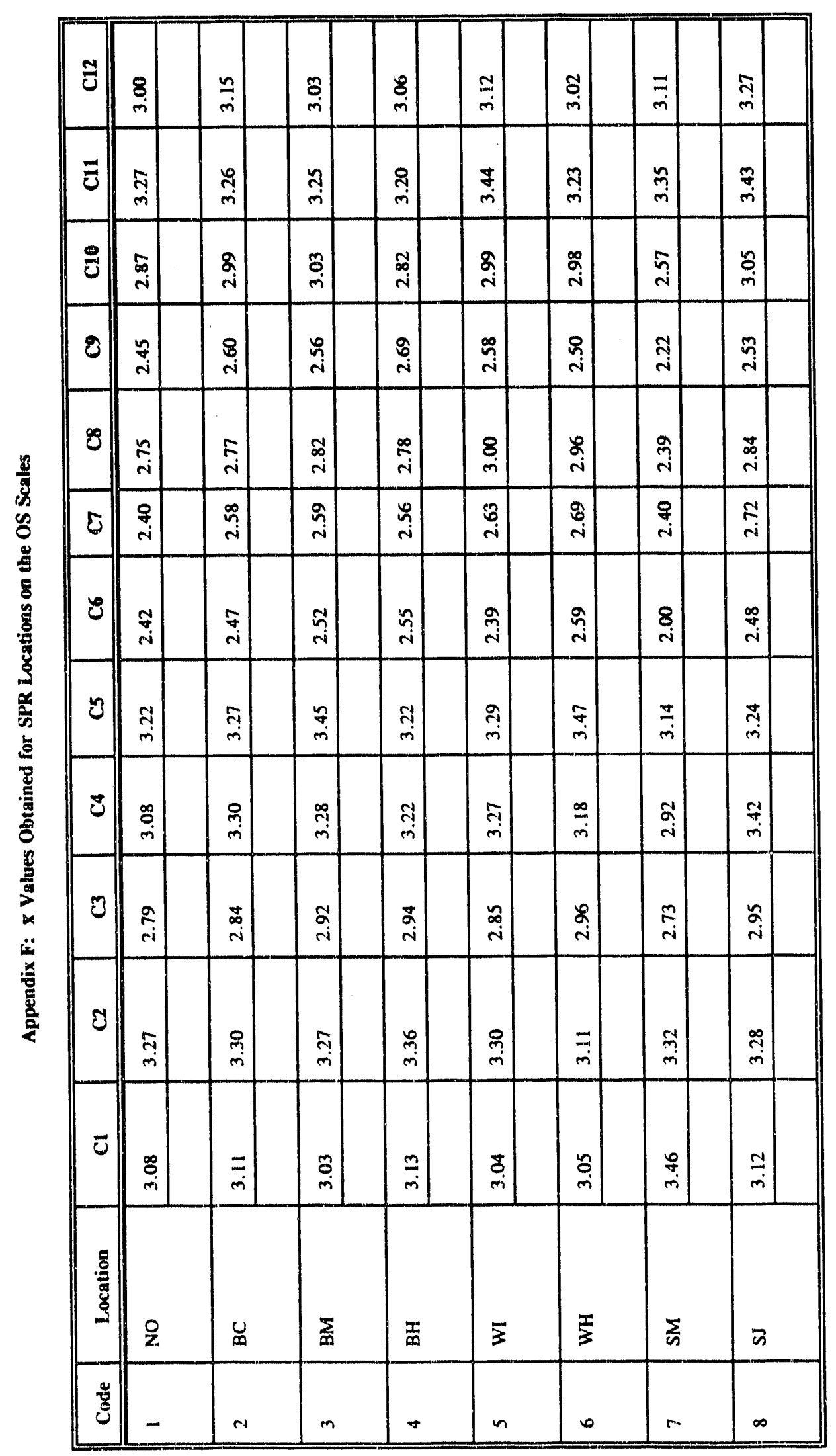




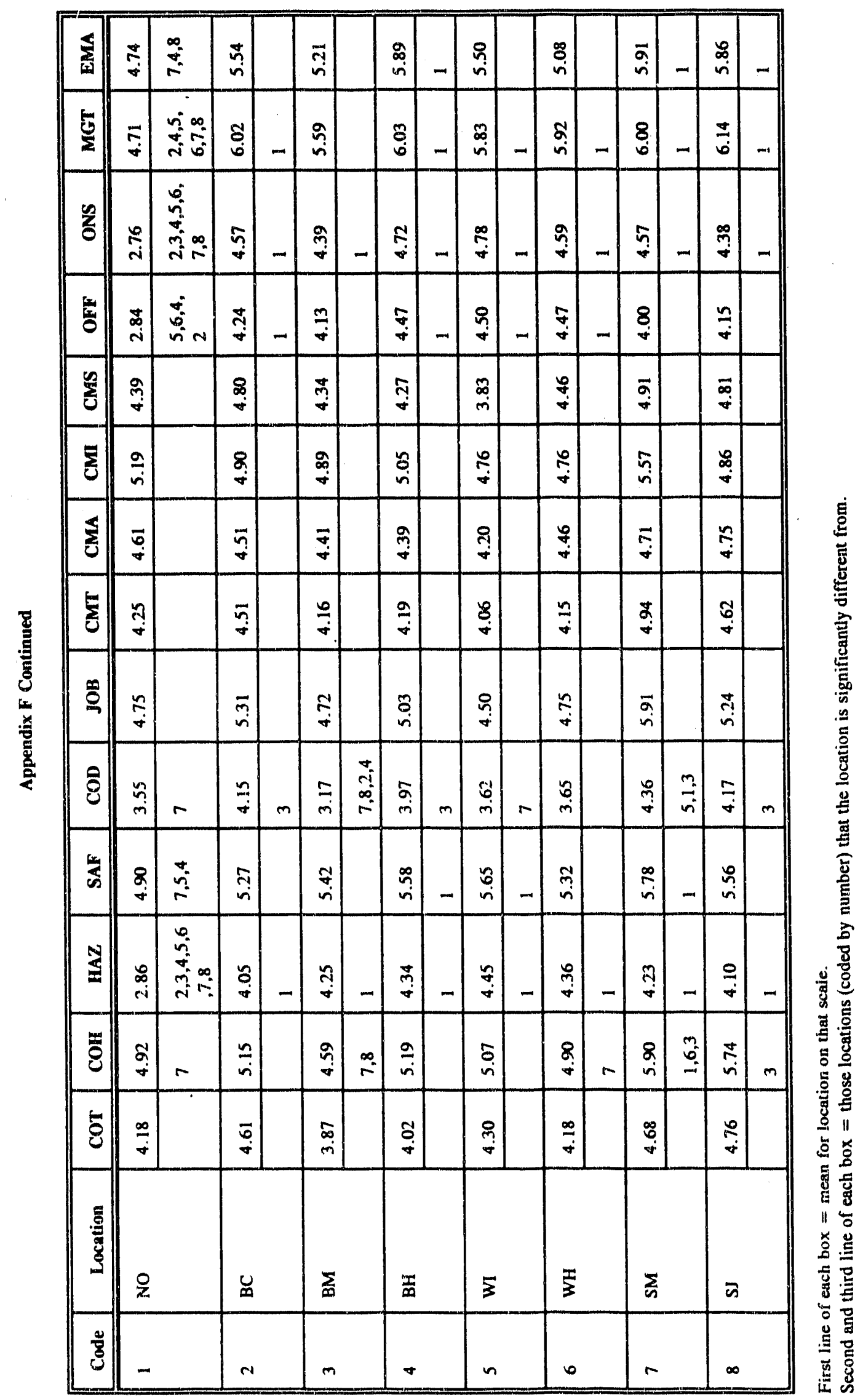




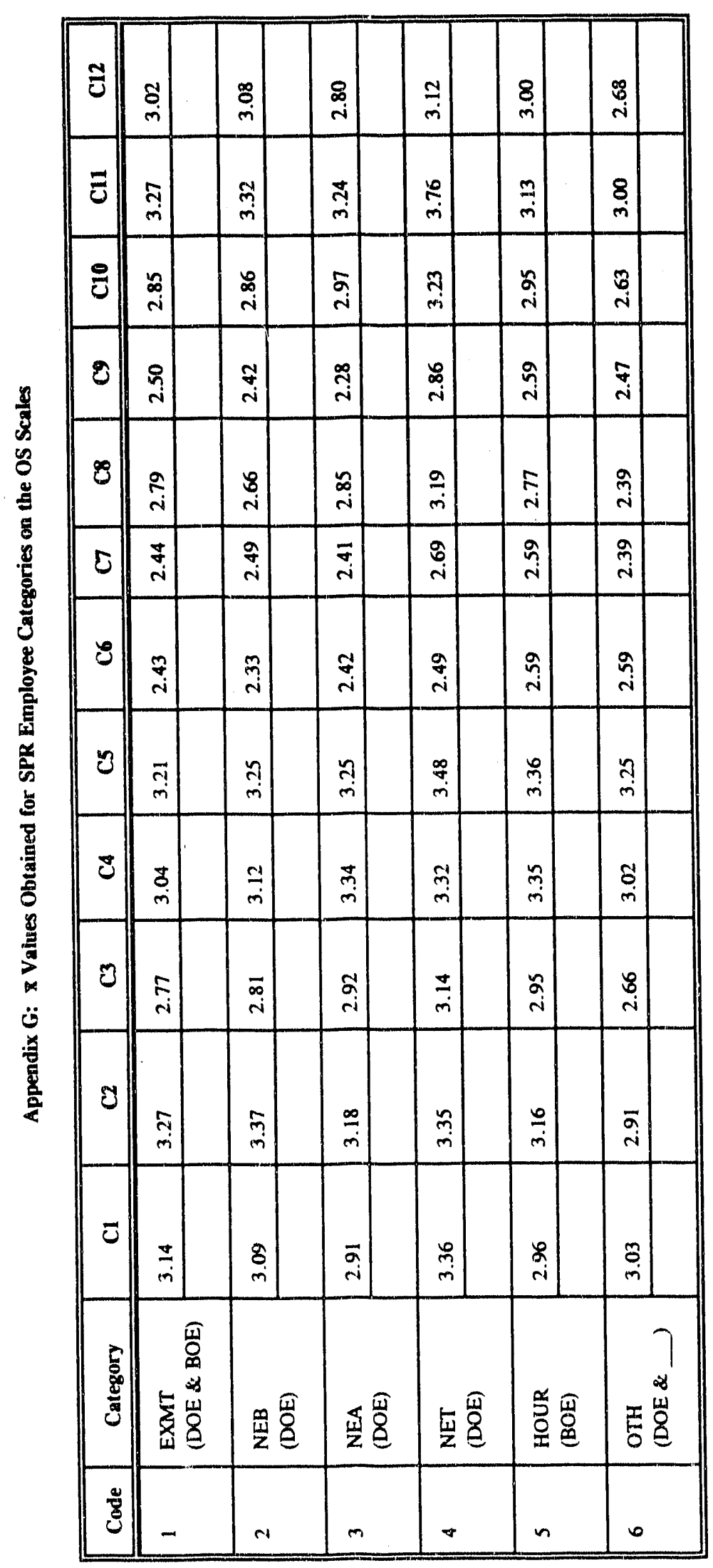




\begin{tabular}{|c|c|c|c|c|c|c|c|c|c|c|c|c|}
\hline 5 & $\underset{+}{5}$ & & $\begin{array}{l}8 \\
n \\
n\end{array}$ & & $\begin{array}{l}\infty \\
\dot{+}\end{array}$ & $n$ & $\begin{array}{l}0 \\
\dot{0} \\
\dot{n}\end{array}$ & & $\begin{array}{l}F \\
\text { n }\end{array}$ & $m$ & $\stackrel{\sim}{\leftarrow}$ & \\
\hline 鸟 & $\begin{array}{l}\tilde{0} \\
\dot{n}\end{array}$ & & $\frac{n}{n}$ & & กี & $n$ & $\stackrel{m}{\dot{m}}$ & & 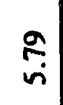 & m & $\stackrel{m}{\hat{n}}$ & \\
\hline$z$ & $\begin{array}{c}7 \\
\cdots \\
\end{array}$ & & $=\bar{m}$ & & $\begin{array}{l}\infty \\
\infty \\
\sim\end{array}$ & $n$ & 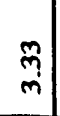 & & 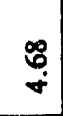 & $m$ & $\stackrel{9}{+}$ & \\
\hline 新 & m & & $\underset{\dot{m}}{\bar{\sigma}}$ & & $\underset{\sim}{*}$ & $n$ & $\stackrel{m}{m}$ & & $\underset{+}{\stackrel{0}{+}}$ & $m$ & $\begin{array}{l}\mathscr{0} \\
\dot{m}\end{array}$ & \\
\hline 胥 & $\stackrel{q}{\dot{v}}$ & & $\stackrel{\tilde{n}}{+}$ & & $\underset{\sigma}{\sigma}$ & & $\stackrel{5}{+}$ & & $\stackrel{8}{*}$ & & $\stackrel{y}{+}$ & \\
\hline 灵 & $\begin{array}{l}\text { సे } \\
\text { n. }\end{array}$ & & $\underset{n}{g}$ & & $\begin{array}{l}\infty \\
\dot{+}\end{array}$ & & $\begin{array}{l}\hat{b} \\
\dot{n}\end{array}$ & $n$ & $\begin{array}{l}0 \\
0 \\
\dot{0}\end{array}$ & + & $\begin{array}{l}\text { ฟ } \\
\text { ஸे }\end{array}$ & \\
\hline$\$$ & $\begin{array}{l}R \\
+\end{array}$ & & $\underset{+}{g}$ & & $\begin{array}{l}\infty \\
0 \\
\dot{\sigma}\end{array}$ & & $\begin{array}{l}\bar{b} \\
\dot{+}\end{array}$ & & $\underset{\sim}{\not}$ & & $\underset{m}{\dot{m}}$ & \\
\hline है & $\begin{array}{l}\stackrel{P}{+} \\
+ \\
+\end{array}$ & & $\stackrel{m}{\underset{\sigma}{*}}$ & & 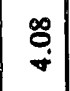 & & $\underset{m}{m}$ & & $\underset{I}{\mathbb{Z}}$ & & ఫ్ & \\
\hline 릉 & $\stackrel{?}{i}$ & & $\begin{array}{c}8 \\
\text { i }\end{array}$ & & $\stackrel{n}{\tilde{r}}$ & & 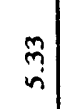 & & $\frac{7}{n}$ & & $\stackrel{\infty}{\infty}$ & \\
\hline 8 & $\begin{array}{l}\infty \\
m \\
m\end{array}$ & & $\stackrel{\vec{n}}{n}$ & & 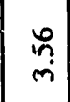 & & $\begin{array}{l}+ \\
\dot{m}\end{array}$ & & $\stackrel{m}{r}$ & & $\underset{m}{+}$ & \\
\hline$\sum_{\infty}^{\infty}$ & $\begin{array}{c}8 \\
4 \\
4 i 5\end{array}$ & & $\frac{a}{n}$ & & $\begin{array}{l}\underset{\infty}{\infty} \\
\dot{+}\end{array}$ & & $\begin{array}{l}P \\
⿱ 亠 \\
\dot{n} \\
n\end{array}$ & & $\underset{n}{\bar{j}}$ & & $\stackrel{m}{+}$ & \\
\hline $\mathbf{3}$ & $\stackrel{n}{m}$ & & $\underset{\dot{m}}{\tilde{n}}$ & $n$ & $\begin{array}{l}\stackrel{a}{0} \\
\text { i }\end{array}$ & $n$ & $\left|\begin{array}{c}\infty \\
\tilde{n} \\
n\end{array}\right|$ & & $\stackrel{\tilde{v}}{\tilde{v}}$ & $\tilde{n}$ & 命 & \\
\hline 官 & $\stackrel{\infty}{\mathfrak{r}}$ & & $\underset{n}{0}$ & & $\stackrel{+}{+}$ & & $\begin{array}{l}5 \\
+\end{array}$ & & $\begin{array}{l}\infty \\
\dot{0} \\
\text { : }\end{array}$ & & $\underset{\sim}{\text { तु }}$ & \\
\hline 8 & $\begin{array}{l}\tilde{H} \\
+\end{array}$ & & $\stackrel{7}{9}$ & & $\stackrel{\infty}{\stackrel{\infty}{m}}$ & & $\begin{array}{l}\tilde{q} \\
\dot{\tau}\end{array} \mid$ & & 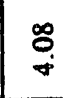 & & $\begin{array}{l}\infty \\
\dot{n}\end{array}$ & \\
\hline $\begin{array}{l}E \\
0 \\
0 \\
0\end{array}$ & 喝 & & 恖 & & 要 & 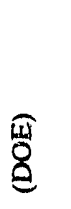 & 5 & & $\begin{array}{l}\text { 똥 } \\
\text { 올 }\end{array}$ & $\begin{array}{l}\text { 푱 } \\
\text { e्e }\end{array}$ & 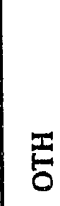 & 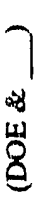 \\
\hline 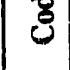 & - & & $\mathrm{A}$ & & $m$ & & $\nabla$ & & $n$ & & 0 & \\
\hline
\end{tabular}




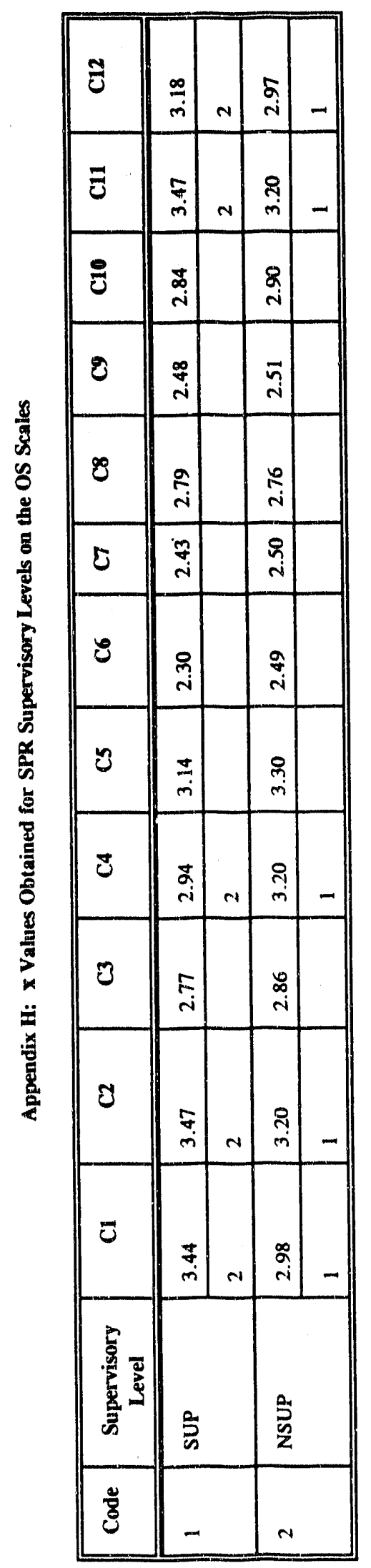

\begin{tabular}{|c|c|c|c|c|}
\hline 起 & ஸे & & $\underset{8}{4}$ & \\
\hline 氙 & $\stackrel{n}{n}$ & & $\frac{=}{i}$ & \\
\hline$z_{0}^{2}$ & $\begin{array}{l}R \\
\dot{r}\end{array} \mid$ & & $\sqrt[5]{n}$ & \\
\hline 농 & $\begin{array}{l}5 \\
\dot{m} \\
m\end{array}$ & & 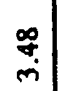 & \\
\hline$\sum^{\infty}$ & $\stackrel{0}{\stackrel{0}{+}}$ & $N$ & $\underset{+}{\stackrel{+}{+}}$ & - \\
\hline 跑 & $\begin{array}{c}8 \\
1\end{array}$ & $N$ & $\vec{\sigma}$ & - \\
\hline 通 & $\begin{array}{l} \pm \\
+ \\
+\end{array}$ & & 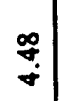 & \\
\hline 胥 & $\begin{array}{l}8 \\
+5\end{array}$ & $N$ & $\stackrel{0}{-0}$ & - \\
\hline 옴 & $\frac{7}{n}$ & & $\stackrel{0}{\leftarrow}$ & \\
\hline 웅 & $\vec{F}$ & & $\begin{array}{c}\mathfrak{b} \\
\dot{m}\end{array}$ & \\
\hline 䊁 & $\begin{array}{l}q \\
\dot{v}\end{array}$ & $N$ & $\underset{5}{5}$ & - \\
\hline$\stackrel{2}{3}$ & $\begin{array}{c}\tilde{b} \\
\dot{m}\end{array}$ & & $\begin{array}{l}\mathscr{H} \\
\dot{r} \\
\dot{m}\end{array}$ & \\
\hline 8 & 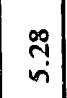 & $\mathrm{A}$ & $\underset{\sigma}{\alpha}$ & - \\
\hline 5 & 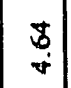 & $N$ & oc & - \\
\hline 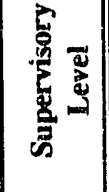 & 寉 & & 窎 & \\
\hline छั & - & & $N$ & \\
\hline
\end{tabular}




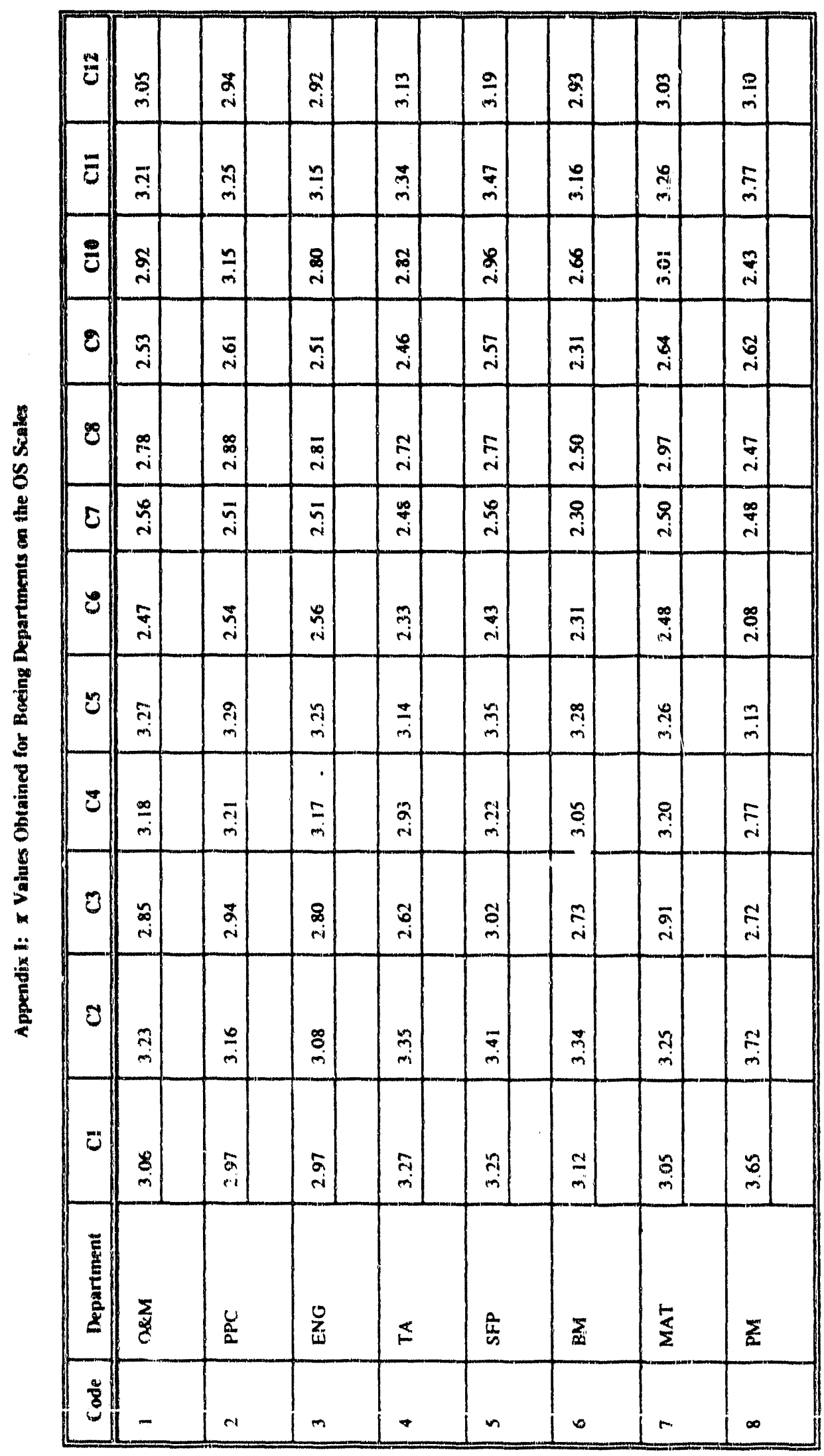




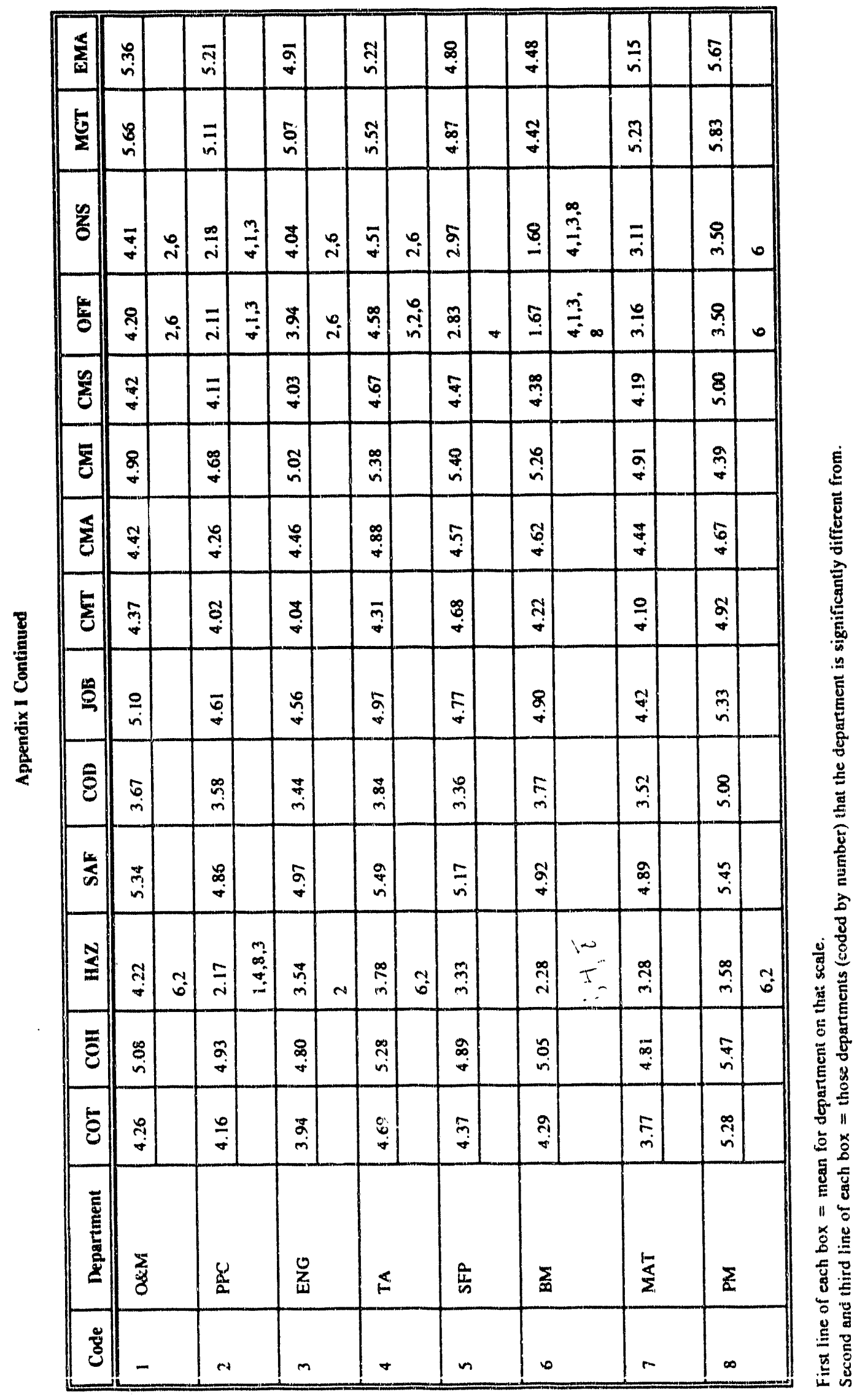




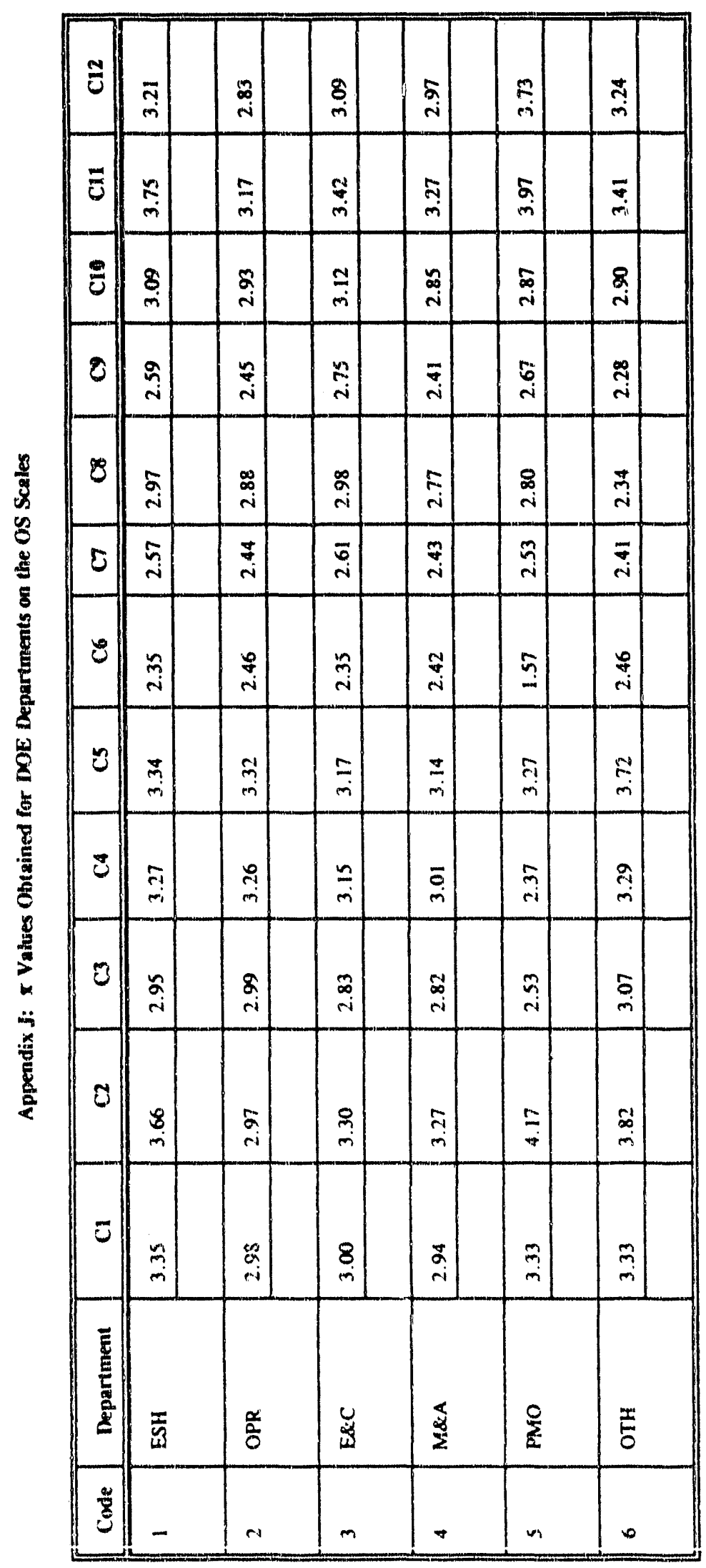




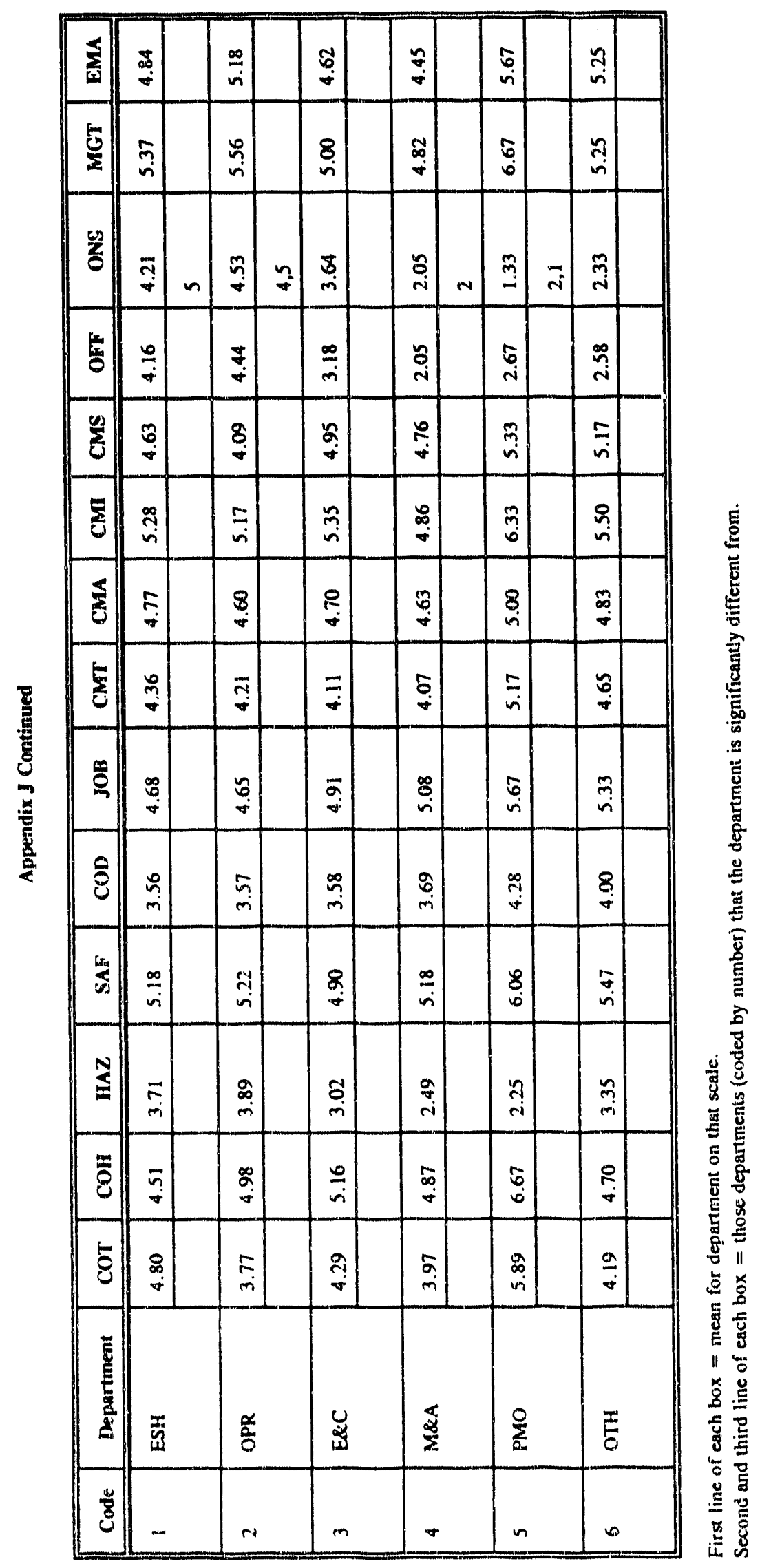




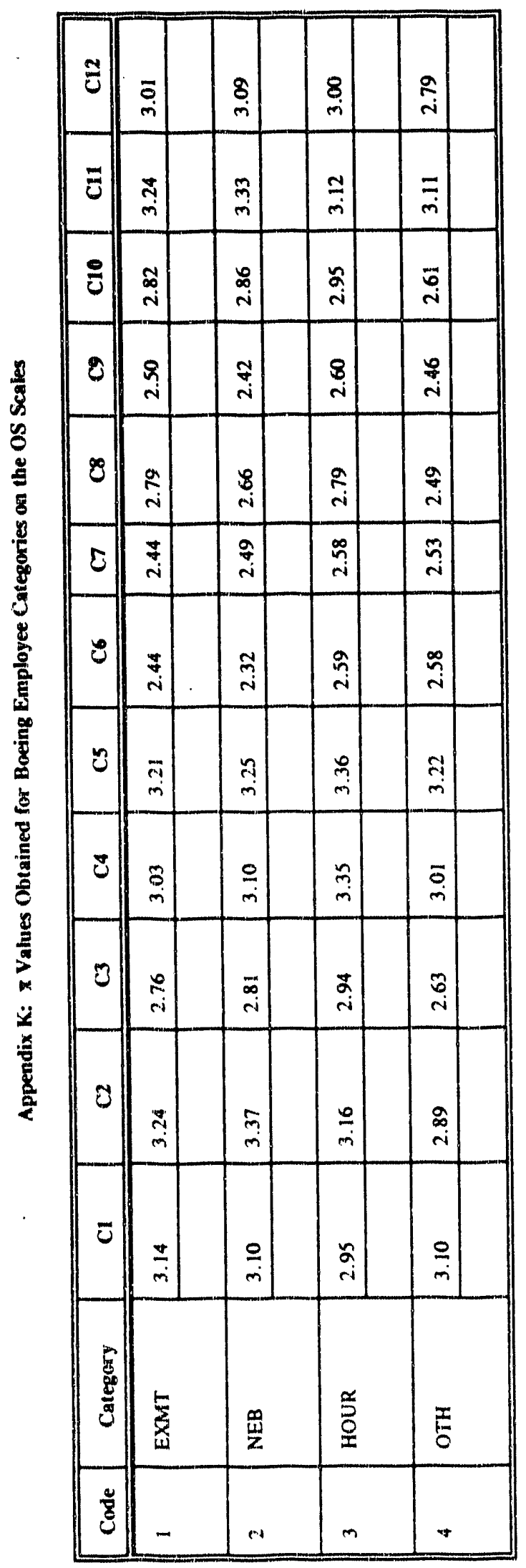

\begin{tabular}{|c|c|c|c|c|c|c|c|c|}
\hline$\sum_{i=2}^{\leq}$ & 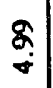 & & ô & & $\stackrel{\infty}{\stackrel{n}{n}}$ & & $\begin{array}{l}a \\
\dot{+} \\
\dot{*}\end{array}$ & \\
\hline$\stackrel{5}{5}$ & $\begin{array}{c}8 \\
\text { जi }\end{array}$ & & $\frac{m}{m}$ & & $\begin{array}{l}\$ \\
\text { wi }\end{array}$ & & 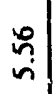 & \\
\hline$\frac{y}{8}$ & $\underset{\dot{m}}{\tilde{m}}$ & & $\stackrel{8}{8}$ & 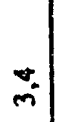 & 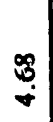 & $N$ & $\underset{\leftarrow}{5}$ & ev \\
\hline 珫 & $\stackrel{f}{-}$ & & $\underset{\sim}{\infty}$ & $m$ & $\underset{+}{\tilde{q}}$ & $e v$ & $=$ & \\
\hline 己ृ & $\underset{+}{+}$ & & $\overrightarrow{\vec{r}}$ & & $\tilde{\mathrm{s}}$ & & $\stackrel{\vec{q}}{+}$ & \\
\hline$\sum$ & $\underset{\sim}{\stackrel{2}{n}}$ & & $\underset{n}{8}$ & 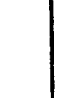 & $\stackrel{5}{0}$ & & 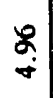 & \\
\hline ș & $\stackrel{\infty}{\leftrightarrow:}$ & & 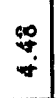 & & $\underset{+}{\tilde{+}}$ & & $\stackrel{5}{+}$ & \\
\hline $\bar{s}$ & $\begin{array}{l}\check{y} \\
\dot{q}\end{array}$ & & $\overrightarrow{\vec{n}}$ & & $\stackrel{i}{+!}$ & & $\stackrel{9}{i}$ & \\
\hline$\stackrel{\circ}{\circ}$ & 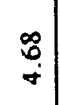 & & 祭 & & $\frac{ \pm}{\dot{n}}$ & & $\overline{\bar{n}}$ & \\
\hline 8 & $\stackrel{\infty}{\stackrel{n}{m}}$ & & $\stackrel{m}{i}$ & & $\stackrel{\vec{n}}{\dot{m}}$ & & $\begin{array}{c}\bar{\sigma} \\
\dot{n}\end{array}$ & \\
\hline$\frac{w}{\infty}$ & $\overrightarrow{\hat{i}}$ & & $\frac{a}{\vec{v}}$ & & 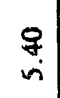 & & $\begin{array}{l}\infty \\
\infty \\
0 \\
\forall\end{array}$ & \\
\hline$\stackrel{N}{\Sigma}$ & $\stackrel{\tilde{m}}{m}$ & $m$ & $\begin{array}{l}5 \\
\dot{m}\end{array}$ & $\ddot{m}$ & 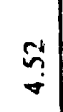 & $\Xi$ & $\frac{ \pm}{\dot{\sigma}}$ & $\mathrm{N}$ \\
\hline $\bar{E}$ & 2 & & $\begin{array}{l}0 \\
\dot{n}\end{array}$ & & 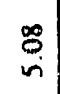 & & $\begin{array}{l}8 \\
\dot{0}\end{array}$ & \\
\hline 8 & $\stackrel{a}{a}$ & & 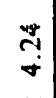 & & $\vec{\square}$ & & $\stackrel{q}{\mathfrak{r}}$ & \\
\hline 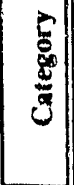 & 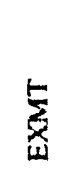 & & $\frac{9}{\mathbf{U}}$ & & 葛 & & $\bar{\xi}$ & \\
\hline 8 & $\cdot$ & & $r$ & & $m$ & & $\forall$ & \\
\hline
\end{tabular}




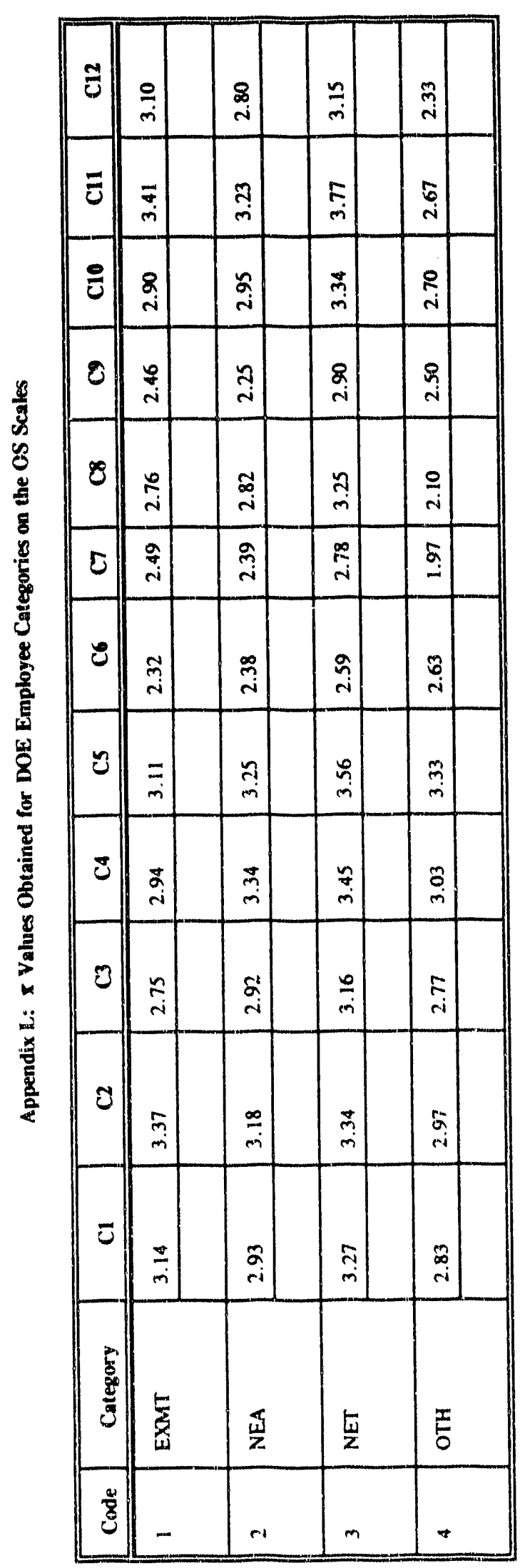

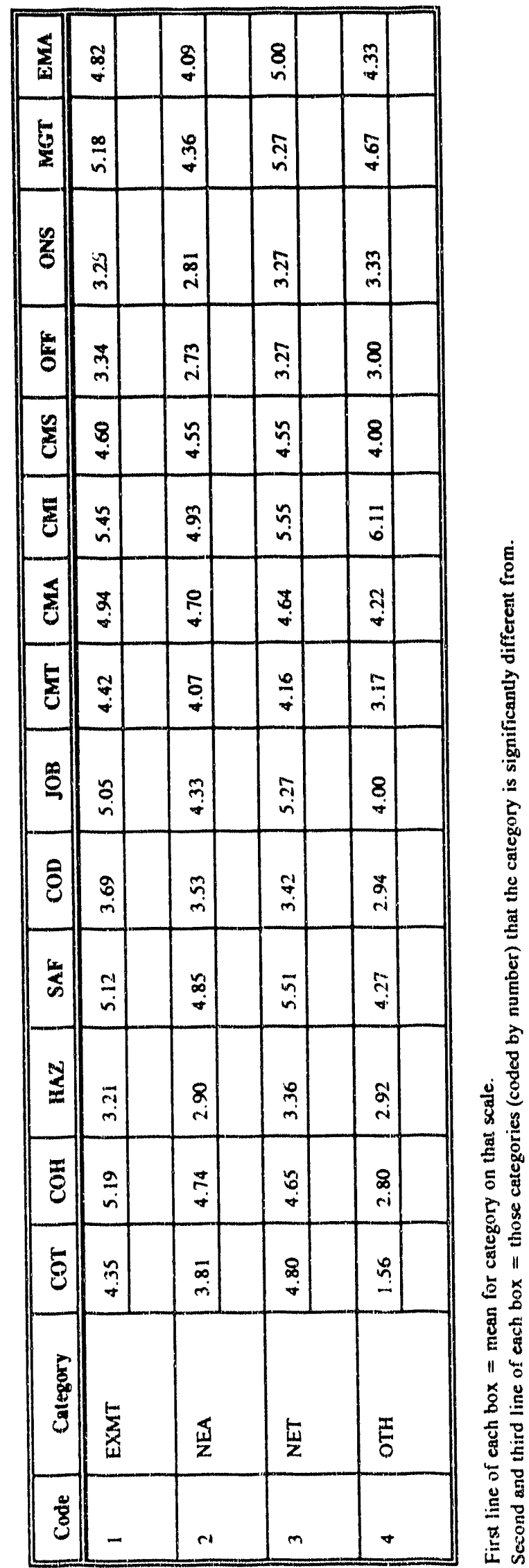




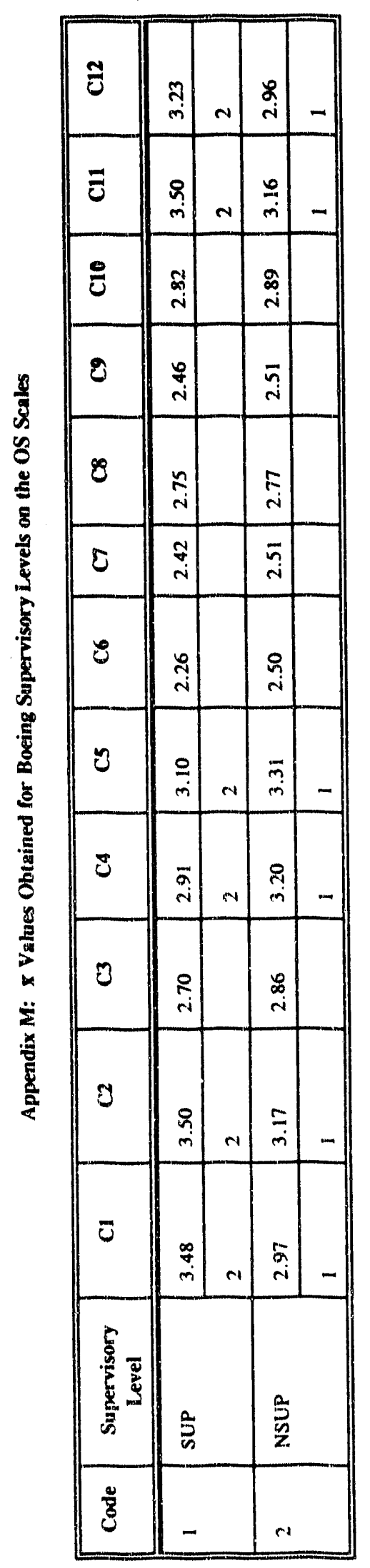

\begin{tabular}{|c|c|c|c|c|}
\hline 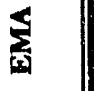 & $\frac{w}{v+}$ & & $\begin{array}{l}\stackrel{\infty}{q} \\
+\end{array}$ & \\
\hline 홀 & $\begin{array}{c}\text { مٌ } \\
\text { vil }\end{array}$ & & $\frac{\infty}{n^{n}}$ & \\
\hline z & $\underset{m}{F}$ & & $\begin{array}{l}8 \\
\dot{m} \\
\end{array}$ & \\
\hline 흥 & $\underset{m}{R}$ & & $\begin{array}{c}\stackrel{+}{n} \\
m\end{array}$ & \\
\hline 窇 & $\begin{array}{l}\infty \\
\dot{\sigma} \\
\dot{\sigma}\end{array}$ & $N$ & $\frac{9}{4}$ & - \\
\hline 를 & $\begin{array}{l}8 \\
\text { ni }\end{array}$ & N & $\stackrel{\substack{\infty \\
\dot{\infty}}}{+}$ & - \\
\hline క్ర & $\stackrel{m}{g}$ & & $\begin{array}{l}\dot{*} \\
\dot{\nabla}\end{array}$ & \\
\hline 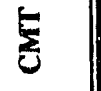 & $\begin{array}{l}\infty \\
\stackrel{+}{+} \\
+\end{array}$ & 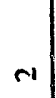 & $\stackrel{\square}{\square}$ & - \\
\hline$\stackrel{\infty}{\circ}$ & 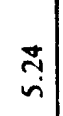 & $N$ & $\underset{+}{\stackrel{+}{*}}$ & - \\
\hline రิ & $\begin{array}{l}F \\
\dot{m}\end{array}$ & & $\begin{array}{l}8 \\
\text { r }\end{array}$ & \\
\hline $\mathfrak{s}^{2}$ & $\begin{array}{l}\overrightarrow{7} \\
\dot{\sim}\end{array}$ & r & $\begin{array}{l}\tilde{y} \\
\dot{n}\end{array}$ & - \\
\hline$\underline{N}$ & $\begin{array}{l}8 \\
7 \\
-1\end{array}$ & & $\stackrel{n}{n}$ & \\
\hline$\overline{8}$ & 节. & $N$ & $\vec{a}$ & - \\
\hline 5 & $\begin{array}{l}\infty \\
0 \\
0\end{array}$ & $N$ & $气$ & - \\
\hline 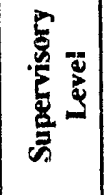 & $\stackrel{2}{S}$ & & $\begin{array}{l}\hat{\vec{n}} \\
\mathbf{z}\end{array}$ & \\
\hline ఫั & - & & C & \\
\hline
\end{tabular}




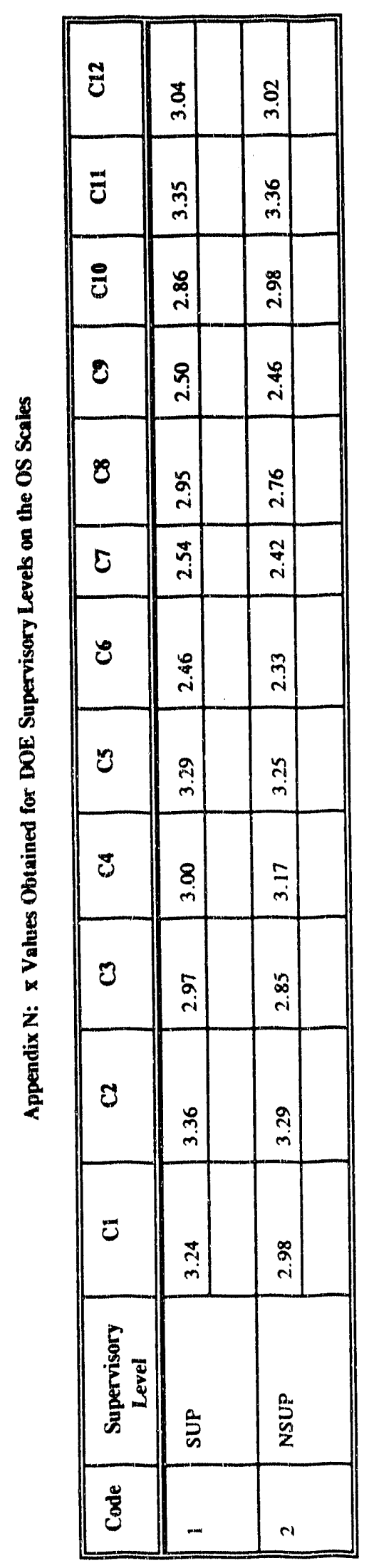

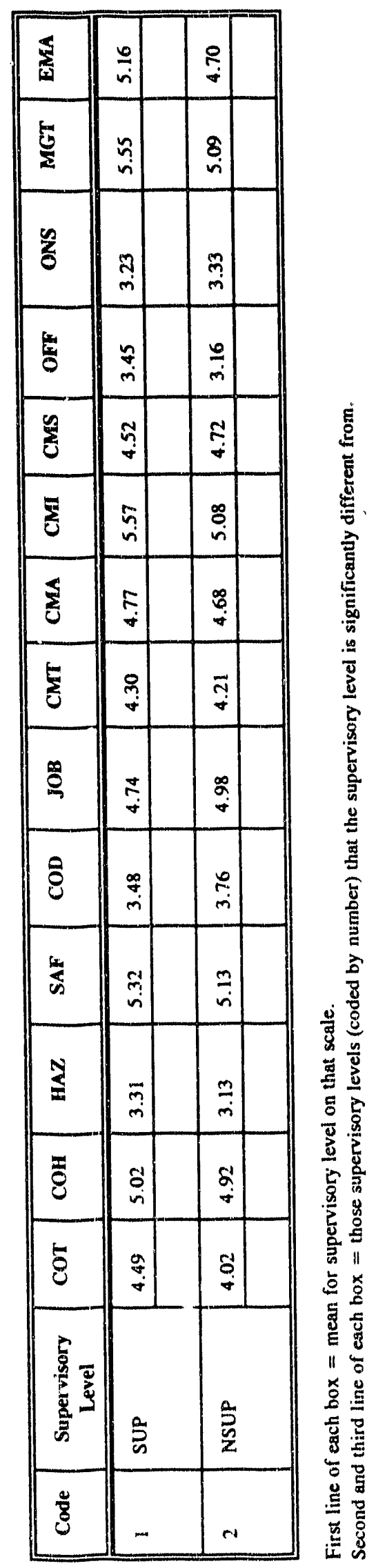



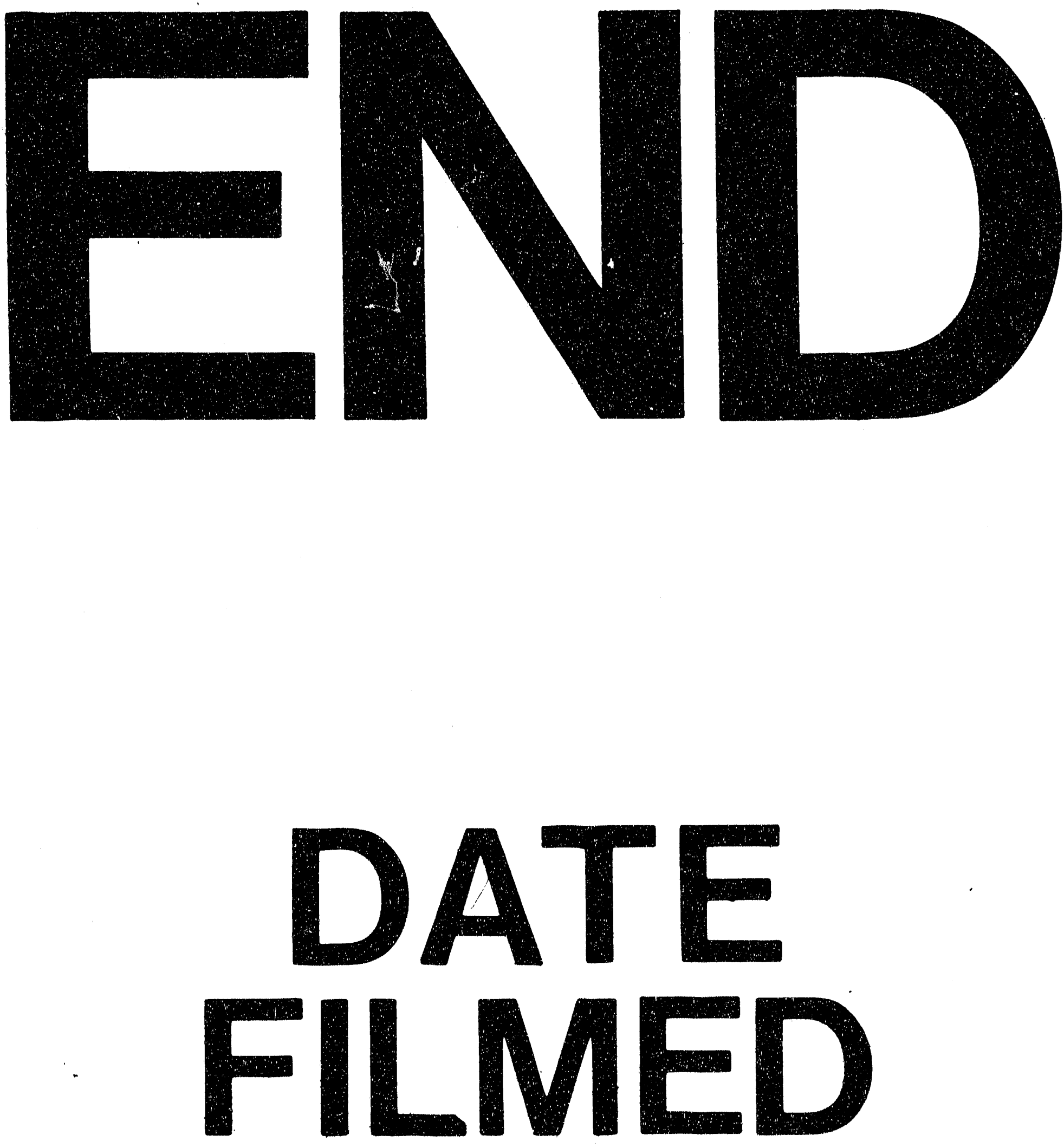

亲

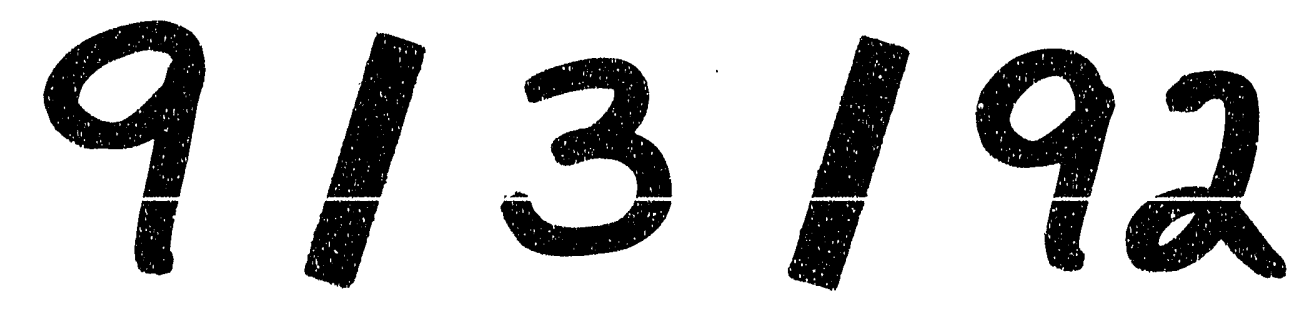

14n-4 
